\title{
Mechanochemical Regulation of Oxidative Addition to a Palladium(0) Bisphosphine Complex
}

Liqi Wang, ${ }^{\dagger}$ Yichen $\mathrm{Yu},{ }^{\dagger}$ Anton O. Razgoniaev, ${ }^{\dagger}$ Patricia N. Johnson, ${ }^{\dagger}$ Chenxu Wang,${ }^{\ddagger}$ Yancong Tian, ${ }^{\ddagger}$ Roman Boulatov, ${ }^{*} \neq$ Stephen L. Craig, ${ }^{*}{ }^{\dagger}$ Ross A. Widenhoefer ${ }^{*, \dagger}$

†Department of Chemistry, Duke University, Durham, North Carolina 27708, USA.

¥Department of Chemistry, University of Liverpool, Crown Street, Liverpool L69 7ZD, UK.

\section{Supporting Information}

\section{Table of Contents}

$\begin{array}{ll}\text { General methods } & \text { S2 }\end{array}$

Synthesis of ligands $\quad$ S3-S10

$\begin{array}{lr}\text { Synthesis of palladium(II) complexes } & \text { S11-S14 }\end{array}$

$\begin{array}{lr}\text { Generation of }(\mathrm{P}-\mathrm{P}) \mathrm{Pd}(0) \text { complexes } & \text { S14-S17 }\end{array}$

Kinetic analysis of oxidative addition to (P-P)Pd(0) complexes S17-S23

Nature of the $(\mathrm{P}-\mathrm{P}) \mathrm{Pd}(0)$ complexes $\quad$ S23-S25

$\begin{array}{lr}\text { DFT calculations } & \text { S25-S28 }\end{array}$

$\begin{array}{ll}\text { Structural comparison of dppe and dppp } & \text { S29 }\end{array}$

$\begin{array}{lr}\text { Crystal structures and data } & \text { S29-S31 }\end{array}$

$\begin{array}{lr}\text { Scans of NMR Spectra } & \text { S32-S91 }\end{array}$

$\begin{array}{lr}\text { References } & \text { S91-S94 }\end{array}$ 
General Methods. All reactions were performed under a nitrogen atmosphere in a flamedried glassware. All solvents used in reaction were dried by Innovative Technologies PureSolv solvent purification system or obtained from Sigma-Aldrich in a Sure/Seal ${ }^{\mathrm{TM}}$ container. ${ }^{1} \mathrm{H},{ }^{31} \mathrm{P}$ and ${ }^{13} \mathrm{C}$ NMR spectra were recorded on Varian INOVA $400 \mathrm{MHz}$, Bruker $500 \mathrm{MHz}$ and Varian INOVA $500 \mathrm{MHz}$ spectrometer at $25^{\circ} \mathrm{C}$ unless otherwise indicated. All ${ }^{1} \mathrm{H}$ and ${ }^{13} \mathrm{C}$ chemical shifts are calibrated using the residual solvent peaks relative to tetramethylsilane (TMS). ${ }^{31} \mathrm{P}$ NMR spectra were obtained with ${ }^{1} \mathrm{H}$ decoupling. Chemical shifts are reported in parts per million $(\delta)$ and coupling constants $(J)$ in Hz. Multiplicities are assigned as singlet (s), doublet (d), triplet (t), quartet $(q)$, multiplet $(m)$, or broad (br). For low temperature kinetic experiments, temperature were determined from a single scan of neat methanol and is accurate to $\pm 1^{\circ} \mathrm{C}$. Phosphoric acid $(85 \%)$ was used as an external standard for ${ }^{31} \mathrm{P}$ NMR measured in the oxidative addition experiments. Force probe ligands $\mathbf{Z}(3,3)^{\mathrm{S} 1}$ and $\mathbf{E}(\mathbf{3}, 3),{ }^{\mathrm{S} 1}$ and $(\mathrm{MeOBiphep}) \mathrm{PdCl}_{2}{ }^{\mathrm{S} 2}$ were synthesized employing published procedures. Z-6,6'-bis(2-hydroxyethyloxy)biindanylidene $(\mathbf{S 1}){ }^{\mathrm{S3}} \quad(R)-\left(6,6^{\prime}\right.$-dihydroxybiphenyl-2,2'-diyl)bis(diphenylphosphine) ${ }^{\mathrm{S4}} \quad$ were $\quad$ synthesized according to published procedures. All other reagents were purchased from major commercial suppliers and were used as received. Error values associated with individual rate constants refer to the standard deviation of the linear regression; error values associated with rate constants depicted in Table 1 refer to the range of the two individual rate constants. 


\section{Synthesis of force probe ligands}

The general synthetic pathway for the synthesis of force probe ligands $\mathbf{Z}(2,2)$ and $\mathbf{E}(\mathbf{2}, \mathbf{2})$ is outlined in Scheme S1.

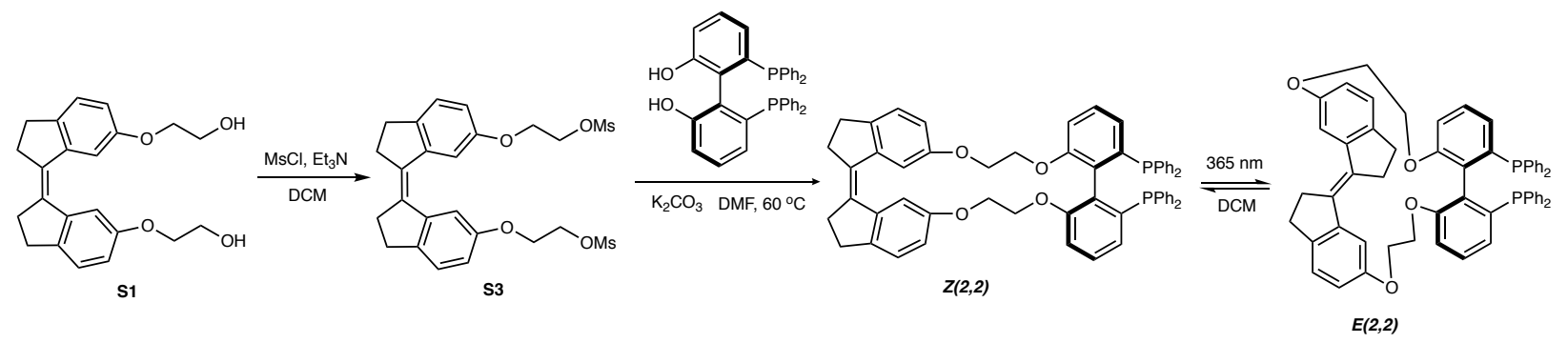

Scheme S1. Synthesis of force probe ligands $Z(2,2)$ and $E(2,2)$.

$\mathbf{Z}(2,2)$. Methanesulfonyl chloride $(0.665 \mathrm{~mL}, 8.60 \mathrm{mmol})$ was added dropwise via syringe to a solution of $\mathbf{S 1}(1.01 \mathrm{~g}, 2.87 \mathrm{mmol})$ and triethylamine $(1.20 \mathrm{~mL}, 8.60 \mathrm{mmol})$ in $\mathrm{CH}_{2} \mathrm{Cl}_{2}(50 \mathrm{~mL})$ at $0{ }^{\circ} \mathrm{C}$ and the resulting solution was stirred for $1 \mathrm{~h}$. The resulting mixture was washed with $1 \mathrm{~N}$ $\mathrm{HCl}(50 \mathrm{~mL})$, water $(100 \mathrm{~mL})$, sat. $\mathrm{NaHCO}_{3}$ (aq.) and brine $(100 \mathrm{~mL})$, dried $\left(\mathrm{Na}_{2} \mathrm{SO}_{4}\right)$, and concentrated under reduced pressure. The residue was chromatographed $\left(\mathrm{SiO}_{2}\right.$ : hexane-ethyl acetate $=1: 1)$ to give $\mathbf{S 3}(1.32 \mathrm{~g}, 90.6 \%)$ as a needle-like off-white crystals.

A suspension of $\mathbf{S} 3(882 \mathrm{mg}, 1.59 \mathrm{mmol})$ and potassium carbonate $(1.33 \mathrm{~g}, 9.62 \mathrm{mmol})$ in DMF (35 mL) was stirred for $1 \mathrm{~h}$ at room temperature. The resulting suspension was then heated at $60{ }^{\circ} \mathrm{C}$, and to this a solution of bisphosphine diol (809 mg, $\left.1.59 \mathrm{mmol}\right)$ in DMF (12 mL) was added over $5 \mathrm{~h}$, and the resulting mixture was stirred for an additional $8 \mathrm{~h}$. The resulting mixture was diluted with $\mathrm{CH}_{2} \mathrm{Cl}_{2}(400 \mathrm{~mL})$ and then washed sequentially with $5 \%$ aqueous, degassed $\mathrm{LiCl}$ $(3 \times 200 \mathrm{~mL})$, water $(2 \times 400 \mathrm{~mL})$, and brine $(400 \mathrm{~mL})$, dried $\left(\mathrm{Na}_{2} \mathrm{SO}_{4}\right)$ and concentrated under reduced pressure. The residue was chromatographed $\left(\mathrm{SiO}_{2}: 5 \%\right.$ ethyl acetate in hexane) to give $\mathbf{Z}(2,2)(554 \mathrm{mg}, 40.0 \%)$ as a white solid. 
For S3: ${ }^{1} \mathrm{H}$ NMR (500 MHz, $\left.\mathrm{CDCl}_{3}\right): \delta 7.22(\mathrm{~d}, J=8.4 \mathrm{~Hz}, 2 \mathrm{H}), 7.16(\mathrm{~d}, J=2.3 \mathrm{~Hz}, 2 \mathrm{H})$, $6.78(\mathrm{dd}, J=8.2,2.3 \mathrm{~Hz}, 2 \mathrm{H}), 4.63-4.57(\mathrm{~m}, 4 \mathrm{H}), 4.32-4.27(\mathrm{~m}, 4 \mathrm{H}), 3.20-3.14(\mathrm{~m}, 4 \mathrm{H}), 3.11$ $(\mathrm{s}, 6 \mathrm{H}), 3.09-3.05(\mathrm{~m}, 4 \mathrm{H}) .{ }^{13} \mathrm{C}\left\{{ }^{1} \mathrm{H}\right\} \operatorname{NMR}\left(126 \mathrm{MHz}, \mathrm{CDCl}_{3}\right): \delta$ 157.20, 144.67, 140.65, 135.99, 125.61, 113.82, 111.27, 68.28, 66.51, 38.00, 32.60, 30.39. HRMS (ESI ${ }^{+}$) calcd (found) for $\mathrm{C}_{24} \mathrm{H}_{28} \mathrm{O}_{8} \mathrm{~S}_{2}[\mathrm{MH}]^{+}: 509.1298$ (509.1287).

For Z(2,2): ${ }^{1} \mathrm{H}$ NMR (500 MHz, $\mathrm{CD}_{2} \mathrm{Cl}_{2}$ ): $\delta 7.56(\mathrm{~s}, 2 \mathrm{H}), 7.40-7.16(\mathrm{~m}, 26 \mathrm{H}), 6.71$ (dd, J $=11.4,8.0 \mathrm{~Hz}, 4 \mathrm{H}), 6.65(\mathrm{dd}, J=8.5,2.2 \mathrm{~Hz}, 2 \mathrm{H}), 3.94(\mathrm{dt}, J=11.7,6.2 \mathrm{~Hz}, 2 \mathrm{H}), 3.85(\mathrm{dt}, J=$ 12.6, $6.2 \mathrm{~Hz}, 2 \mathrm{H}), 3.77(\mathrm{dt}, J=9.6,4.9 \mathrm{~Hz}, 2 \mathrm{H}), 3.43(\mathrm{dt}, J=10.5,4.8 \mathrm{~Hz}, 2 \mathrm{H}), 2.98-2.75(\mathrm{~m}$, $8 \mathrm{H}) .{ }^{13} \mathrm{C}\left\{{ }^{1} \mathrm{H}\right\} \mathrm{NMR}\left(126 \mathrm{MHz}, \mathrm{CD}_{2} \mathrm{Cl}_{2}\right): \delta 157.44,157.01,156.97,156.92,141.86,141.35,139.98$, 139.96, 139.94, 139.16, 139.11, 139.10, 139.04, 137.35, 137.31, 137.26, 135.94, 135.18, 135.09, $135.00,133.69,133.60,133.52,132.62,132.47,132.32,129.04,128.91,128.64,128.62,128.60$, $128.42,128.40,128.36,113.90,111.04,109.66,66.16,65.65,35.66,30.03 .{ }^{31} \mathrm{P} N M R(202 \mathrm{MHz}$, $\mathrm{CD}_{2} \mathrm{Cl}_{2}$ ): $\delta$-13.22. HRMS (ESI ${ }^{+}$) calcd (found) for $\mathrm{C}_{58} \mathrm{H}_{48} \mathrm{O}_{4} \mathrm{P}_{2}[\mathrm{MH}]^{+}: 871.3101$ (871.3099).

$\mathbf{E}(\mathbf{2 , 2})$. A solution of $\mathbf{Z}(\mathbf{2 , 2})(313 \mathrm{mg}, 0.359 \mathrm{mmol})$ in $\mathrm{CH}_{2} \mathrm{Cl}_{2}(300 \mathrm{~mL})$ was sparged with nitrogen for $20 \mathrm{~min}$ and then irradiated $(\lambda=365 \mathrm{~nm})$ for $1 \mathrm{~h}$ with vigorous stirring (photo-stationary state: $96 \% Z(2,2)$ and $4 \% E(2,2))$. The solvent was evaporated under vacuum and the residue was chromatographed $\left(\mathrm{SiO}_{2}: 10 \%\right.$ ethyl acetate in hexane) to give $\mathbf{E}(\mathbf{2}, \mathbf{2})(10.4 \mathrm{mg}, 3.3 \%$ yield $)$ as a white solid. ${ }^{1} \mathrm{H}$ NMR $\left(400 \mathrm{MHz}, \mathrm{CDCl}_{3}\right): \delta 7.33-7.23(\mathrm{~m}, 6 \mathrm{H}), 7.24(\mathrm{~d}, J=4.9 \mathrm{~Hz}, 1 \mathrm{H}), 7.2$ (dd, J = 5.0, 2.8 Hz, 3H), $7.14(\mathrm{~d}, J=8.0 \mathrm{~Hz}, 2 \mathrm{H}), 7.06(\mathrm{t}, J=7.3 \mathrm{~Hz}, 2 \mathrm{H}), 7.04-6.93(\mathrm{~m}, 8 \mathrm{H})$, $6.91(\mathrm{~d}, J=8.2 \mathrm{~Hz}, 2 \mathrm{H}), 6.85(\mathrm{dd}, J=7.6,3.2 \mathrm{~Hz}, 2 \mathrm{H}), 6.67-6.54(\mathrm{~m}, 6 \mathrm{H}), 4.45-4.22(\mathrm{~m}, 4 \mathrm{H})$, $3.86-3.61(\mathrm{~m}, 4 \mathrm{H}), 3.06-2.55(\mathrm{~m}, 8 \mathrm{H}) .{ }^{31} \mathrm{P} \mathrm{NMR}\left(162 \mathrm{MHz}, \mathrm{CDCl}_{3}\right): \delta-10.71 . \mathrm{HRMS}\left(\mathrm{ESI}^{+}\right)$ calcd (found) for $\mathrm{C}_{58} \mathrm{H}_{48} \mathrm{O}_{4} \mathrm{P}_{2}[\mathrm{MH}]^{+}: 871.3101$ (871.3098).

Ligands $\mathbf{Z}(2,3)$ and $E(2,3)$ were synthesized from 6-hydroxyl-1-indanone employing the sequence depicted in Scheme S2. 

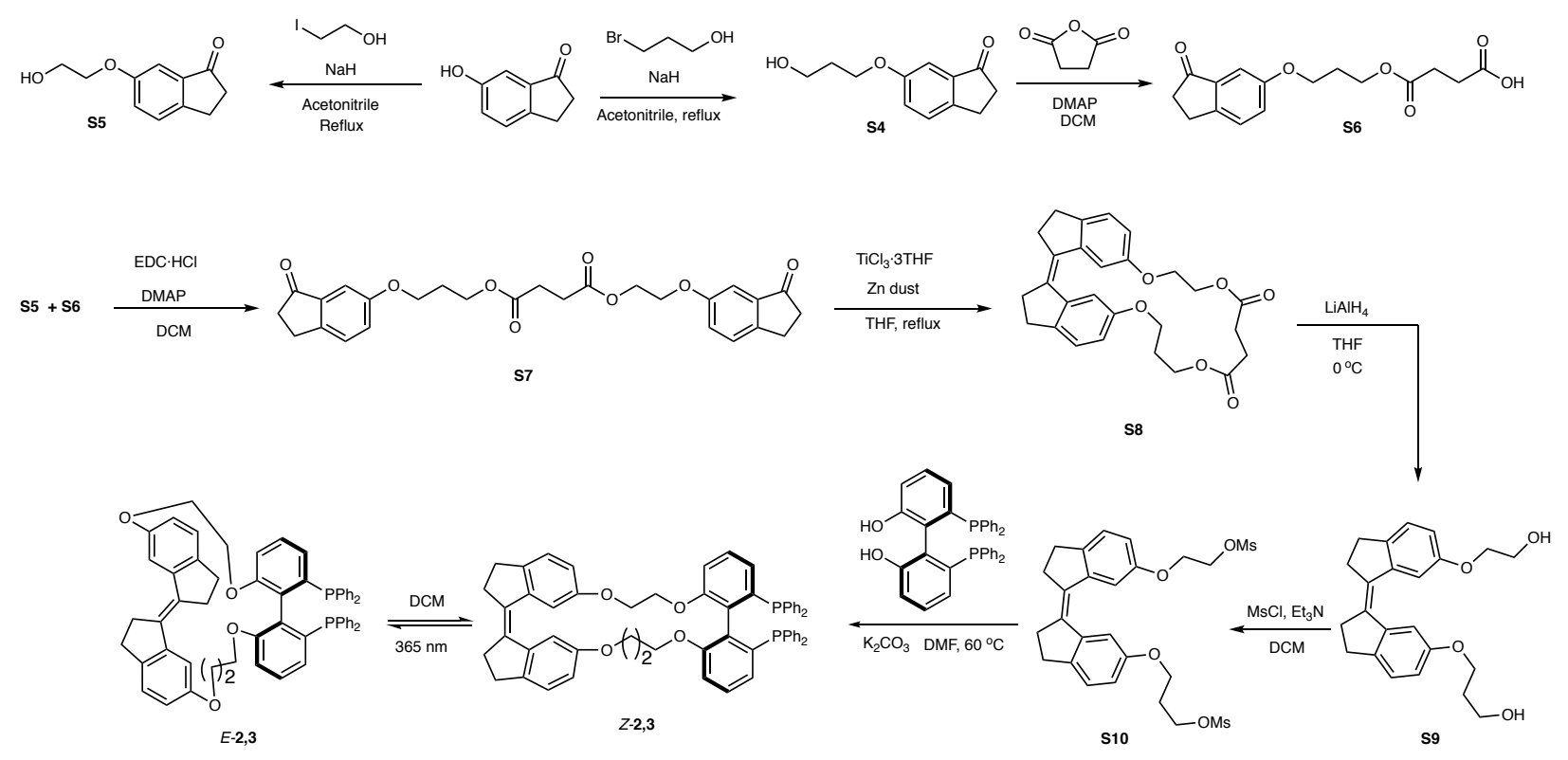

Scheme S2. Synthesis of ligands $Z(2,3)$ and $E(2,3)$.

6-(3-Hydroxypropyloxy)indanone (S4). Sodium hydride $(0.970 \mathrm{~g}, 40.4 \mathrm{mmol})$ was added to a solution of 6-hydroxyl-1-indanone $(5.00 \mathrm{~g}, 33.7 \mathrm{mmol})$ in acetonitrile $(30 \mathrm{~mL})$ at room temperature. The resulting suspension was heated at $80^{\circ} \mathrm{C}$, and 3-bromo-1-propanol $(6.10 \mathrm{~mL}$, $67.5 \mathrm{mmol}$ ) was added dropwise. The reaction mixture was heated at reflux overnight, cooled to room temperature, and solvent was evaporated under reduced pressure. The resulting orange slurry was dissolved in $\mathrm{CH}_{2} \mathrm{Cl}_{2}(100 \mathrm{~mL})$ and the resulting solution was washed with $1 \mathrm{~N} \mathrm{HCl}(50$ $\mathrm{mL})$, water $(100 \mathrm{~mL})$, and brine $(100 \mathrm{~mL})$, dried $\left(\mathrm{Na}_{2} \mathrm{SO}_{4}\right)$, and concentrated under vacuum. The resulting residue was chromatographed $\left(\mathrm{SiO}_{2}\right.$ : hexane-ethyl acetate $\left.=1: 1\right)$ to give $\mathbf{S 4}(5.82 \mathrm{~g}$, $84 \%)$ as an amorphous white solid. ${ }^{1} \mathrm{H} \mathrm{NMR}\left(500 \mathrm{MHz}, \mathrm{CDCl}_{3}\right): \delta 7.34(\mathrm{~d}, \mathrm{~J}=8.2 \mathrm{~Hz}, 1 \mathrm{H}), 7.20$ $-7.14(\mathrm{~m}, 2 \mathrm{H}), 4.13(\mathrm{t}, J=6.0 \mathrm{~Hz}, 2 \mathrm{H}), 3.85(\mathrm{q}, J=5.7 \mathrm{~Hz}, 2 \mathrm{H}), 3.08-3.01(\mathrm{~m}, 2 \mathrm{H}), 2.73-2.66$ (m, 2H), $2.08-2.02(\mathrm{~m}, 2 \mathrm{H}), 1.99(\mathrm{t}, J=5.3 \mathrm{~Hz}, 1 \mathrm{H}) .{ }^{13} \mathrm{C}\left\{{ }^{1} \mathrm{H}\right\} \mathrm{NMR}(126 \mathrm{MHz}, \mathrm{CDCl}): \delta 207.22$, $\left.158.64,148.20,138.30,127.50,124.38,105.87,66.02,60.24,37.10,32.02,25.22 . \mathrm{HRMS}_{(\mathrm{ESI}}{ }^{+}\right)$ calcd (found) for $\mathrm{C}_{12} \mathrm{H}_{15} \mathrm{O}_{3}\left(\mathrm{MH}^{+}\right): 207.1016$ (207.1017). 
6-(2-Hydroxyethyloxy)indanone (S5). Sodium hydride $(0.710 \mathrm{~g}, 29.6 \mathrm{mmol})$ was added to a solution of 6-hydroxyl-1-indanone $(3.36 \mathrm{~g}, 22.7 \mathrm{mmol})$ in THF $(50 \mathrm{~mL})$ at room temperature. The resulting suspension was heated at $70{ }^{\circ} \mathrm{C}$ and 2-iodo-1-ethanol $(2.83 \mathrm{~mL}, 36.3 \mathrm{mmol})$ was added dropwise. The reaction mixture was heated at reflux overnight, cooled to room temperature, and concentrated under vacuum. The resulting orange slurry was dissolved in $\mathrm{CH}_{2} \mathrm{Cl}_{2}(100 \mathrm{~mL})$, and the resulting solution was washed with $1 \mathrm{~N} \mathrm{HCl}(50 \mathrm{~mL})$, water $(100 \mathrm{~mL})$, and brine $(100 \mathrm{~mL})$, dried $\left(\mathrm{Na}_{2} \mathrm{SO}_{4}\right)$, and concentrated under vacuum. The residue was chromatographed $\left(\mathrm{SiO}_{2}\right.$ : hexane-ethyl acetate $\left.=1: 3\right)$ to give $\mathbf{S 5}(1.58 \mathrm{~g}, 36.2 \%)$ as an amorphous white solid. ${ }^{1} \mathrm{H}$ NMR $\left(500 \mathrm{MHz}, \mathrm{CDCl}_{3}\right)$ : $\delta 7.35(\mathrm{~d}, J=8.3 \mathrm{~Hz}, 1 \mathrm{H}), 7.22-7.15(\mathrm{~m}$, 2H), $4.09(\mathrm{t}, J=4.5 \mathrm{~Hz}, 2 \mathrm{H}), 3.97(\mathrm{t}, J=4.5 \mathrm{~Hz}, 2 \mathrm{H}), 3.08-3.02(\mathrm{~m}, 2 \mathrm{H}), 2.73-2.66(\mathrm{~m}, 2 \mathrm{H})$, $2.28(\mathrm{~s}, 1 \mathrm{H}) .{ }^{13} \mathrm{C}\left\{{ }^{1} \mathrm{H}\right\} \mathrm{NMR}\left(126 \mathrm{MHz}, \mathrm{CDCl}_{3}\right): \delta 207.18,158.48,148.44,138.30,127.59,124.38$, 105.91, 69.68, 61.36, 37.08, 25.22. HRMS (ESI ${ }^{+}$) calcd (found) for $\mathrm{C}_{11} \mathrm{H}_{13} \mathrm{O}_{3}[\mathrm{MH}]^{+}: 193.0859$ (193.0858).

4-0xo-4-(3-((3-oxo-2,3-dihydro-1H-inden-5-yl)oxy)propoxy)butanoic acid (S6). A solution of S4 (3.65 g, $17.7 \mathrm{mmol})$, succinic anhydride $(2.13,21.2 \mathrm{mmol})$, and 4dimethylaminopyridine (DMAP, $0.216 \mathrm{~g}, 1.77 \mathrm{mmol}$ ) in $\mathrm{CH}_{2} \mathrm{Cl}_{2}(50 \mathrm{~mL}$ ) was stirred overnight at room temperature. The resulting mixture was washed with $1 \mathrm{~N} \mathrm{HCl}(50 \mathrm{~mL})$, water $(3 \times 50 \mathrm{~mL})$, and brine $(50 \mathrm{~mL})$, dried $\left(\mathrm{Na}_{2} \mathrm{SO}_{4}\right)$, and concentrated under vacuum. The residue was chromatographed $\left(\mathrm{SiO}_{2}\right.$ : hexane-ethyl acetate $\left.=1: 3\right)$ to yield $\mathbf{S 6}(4.62 \mathrm{~g}, 85.3 \%)$ as a needle-like white crystal. ${ }^{1} \mathrm{H}$ NMR $\left(500 \mathrm{MHz}, \mathrm{CDCl}_{3}\right): \delta 9.44(\mathrm{br} \mathrm{s}, 1 \mathrm{H}), 7.35(\mathrm{~d}, J=8.3 \mathrm{~Hz}, 1 \mathrm{H}), 7.22-7.13$ $(\mathrm{m}, 2 \mathrm{H}), 4.29(\mathrm{t}, J=6.1 \mathrm{~Hz}, 2 \mathrm{H}), 4.07(\mathrm{t}, J=6.3 \mathrm{~Hz}, 2 \mathrm{H}), 3.11-2.99(\mathrm{~m}, 2 \mathrm{H}), 2.76-2.59(\mathrm{~m}, 6 \mathrm{H})$, $2.11(p, J=6.2 \mathrm{~Hz}, 2 \mathrm{H}) .{ }^{13} \mathrm{C}\left\{{ }^{1} \mathrm{H}\right\} \mathrm{NMR}\left(126 \mathrm{MHz}, \mathrm{CDCl}_{3}\right): \delta 207.95,177.12,172.29,158.59$, $148.48,138.14,127.53,124.90,105.79,64.84,61.55,37.11,29.08,28.99,28.55,25.27$. HRMS (ESI) calcd (found) for $\mathrm{C}_{16} \mathrm{H}_{19} \mathrm{O}_{6}[\mathrm{M}+\mathrm{H}]^{+}: 307.1176$ (307.1179).

(2-(Indanon-6-oxy)ethyl) (3-(indanon-6-oxy)propyl)succinate (S7). A solution of S5 (1.58 g, $8.22 \mathrm{mmol})$, $\mathbf{S 6}(2.75 \mathrm{~g}, 8.97 \mathrm{mmol})$, DMAP $(0.55 \mathrm{~g}, 4.52 \mathrm{mmol})$, and $\mathrm{N}$-(3- 
dimethylaminopropyl)- $N$ '-ethylcarbodiimide hydrochloride $(\mathrm{EDC} \cdot \mathrm{HCl}, 2.58 \mathrm{~g}, 13.5 \mathrm{mmol})$ in $\mathrm{CH}_{2} \mathrm{Cl}_{2}(30 \mathrm{~mL})$ was stirred at room temperature overnight. The resulting mixture was washed with $1 \mathrm{~N} \mathrm{HCl}(50 \mathrm{~mL})$, water $(50 \mathrm{~mL})$, and brine $(50 \mathrm{~mL})$, and concentrated under vacuum. The residue was chromatographed $\left(\mathrm{SiO}_{2}\right.$ : hexane-ethyl acetate $\left.=1: 3\right)$ to give $\mathbf{S} 7$ in $(3.45 \mathrm{~g}, 87.3 \%)$ as a colorless oil. ${ }^{1} \mathrm{H}$ NMR $\left(500 \mathrm{MHz}, \mathrm{CDCl}_{3}\right): \delta 7.34(\mathrm{~d}, J=5.7 \mathrm{~Hz}, 1 \mathrm{H}), 7.32(\mathrm{~d}, J=6.9 \mathrm{~Hz}, 1 \mathrm{H})$, $7.19-7.10(\mathrm{~m}, 4 \mathrm{H}), 4.44-4.40(\mathrm{~m}, 2 \mathrm{H}), 4.25(\mathrm{t}, J=6.3 \mathrm{~Hz}, 2 \mathrm{H}), 4.17-4.14(\mathrm{~m}, 2 \mathrm{H}), 4.02(\mathrm{t}, J$ $=6.2 \mathrm{~Hz}, 2 \mathrm{H}), 3.06-2.99(\mathrm{~m}, 4 \mathrm{H}), 2.70-2.61(\mathrm{~m}, 8 \mathrm{H}), 2.09(\mathrm{p}, J=6.2 \mathrm{~Hz}, 2 \mathrm{H}) .{ }^{13} \mathrm{C}\left\{{ }^{1} \mathrm{H}\right\} \mathrm{NMR}$ (126 MHz, $\left.\mathrm{CDCl}_{3}\right): \delta 206.97,206.82,172.20,172.18,158.50,158.20,148.41,148.12,138.24$, $138.23,127.58,127.48,124.38,105.84,105.58,66.22,64.67,62.91,61.51,37.01,29.05,29.02$, 28.50, 25.15. HRMS (ESI ${ }^{+}$) calcd (found) for $\mathrm{C}_{27} \mathrm{H}_{29} \mathrm{O}_{8}[\mathrm{MH}]^{+}: 481.1779$ (481.1865).

S8. THF $(500 \mathrm{~mL})$ was added to a mixture of $\operatorname{TiCl}_{3} \cdot 3 \operatorname{THF}(10 \mathrm{~g}, 27.0 \mathrm{mmol})$ and zinc dust (20 g, $306 \mathrm{mmol}$ ) at room temperature and the resulting suspension was stirred vigorously at 70 ${ }^{\circ} \mathrm{C}$ for $1.5 \mathrm{~h}$. A solution of $\mathbf{S} 7(3.40 \mathrm{~g}, 7.08 \mathrm{mmol})$ in THF $(250 \mathrm{~mL})$ was added to the resulting suspension over the course of 5-7 $\mathrm{h}$ via addition funnel and the resulting mixture was heated at reflux overnight. The reaction mixture was cooled to room temperature and quenched with sat. aqueous $\mathrm{NH}_{4} \mathrm{Cl}(500 \mathrm{~mL})$. The resulting suspension was filtered, the layers were separated, and the organic fraction was concentrated under reduced pressure. The resulting residue was dissolved in $\mathrm{CH}_{2} \mathrm{Cl}_{2}(300 \mathrm{~mL})$ and the resulting solution was washed with water $(300 \mathrm{~mL})$, and brine $(300 \mathrm{~mL})$, dried $\left(\mathrm{Na}_{2} \mathrm{SO}_{4}\right)$ and concentrated under vacuum. The residue was chromatographed $\left(\mathrm{SiO}_{2}\right.$ : hexane-ethyl acetate $\left.=1: 1\right)$ to give $\mathbf{S 8}(2.59 \mathrm{~g}, 81.6 \%)$ as an amorphous yellow solid. ${ }^{1} \mathrm{H}$ NMR $\left(500 \mathrm{MHz}, \mathrm{CDCl}_{3}\right)$ : $\delta 7.63(\mathrm{~d}, J=2.4 \mathrm{~Hz}, 1 \mathrm{H}), 7.62(\mathrm{~d}, J=2.4 \mathrm{~Hz}, 1 \mathrm{H}), 7.20$ (d, J = 7.7 Hz, 1H), $7.18(\mathrm{~d}, J=7.7 \mathrm{~Hz}, 1 \mathrm{H}), 6.78(\mathrm{dt}, J=8.3,2.1 \mathrm{~Hz}, 2 \mathrm{H}), 4.41(\mathrm{t}, J=4.8 \mathrm{~Hz}, 2 \mathrm{H})$, $4.31(\mathrm{t}, J=5.6 \mathrm{~Hz}, 2 \mathrm{H}), 4.06(\mathrm{t}, J=4.8 \mathrm{~Hz}, 2 \mathrm{H}), 4.01(\mathrm{t}, J=6.0 \mathrm{~Hz}, 2 \mathrm{H}), 2.96-2.90(\mathrm{~m}, 4 \mathrm{H}), 2.85$ $-2.79(\mathrm{~m}, 4 \mathrm{H}), 2.71-2.63(\mathrm{~m}, 4 \mathrm{H}), 2.08(\mathrm{p}, J=5.8 \mathrm{~Hz}, 2 \mathrm{H}) .{ }^{13} \mathrm{C}\left\{{ }^{1} \mathrm{H}\right\} \mathrm{NMR}\left(126 \mathrm{MHz}, \mathrm{CDCl}_{3}\right): \delta$ $181.82,172.02,171.85,157.31,157.00,141.88,141.63,141.37,141.07,135.89,135.30,125.95$, 
$125.91,116.37,114.93,108.76,107.95,66.25,64.53,63.31,61.56,35.60,35.55,30.02,29.79$ 29.68, 29.03. HRMS (ESI) calcd (found) for $\mathrm{C}_{27} \mathrm{H}_{29} \mathrm{O}_{6}[\mathrm{MH}]^{+}: 449.1959$ (449.1963).

S9. ${ }^{\mathrm{S}} \mathrm{LiAlH}_{4}$ (342 mg, 9 mmol) was added slowly to a stirred solution of $\mathbf{S 8}$ (2g, 4.45 $\mathrm{mmol})$ in THF $(150 \mathrm{~mL})$ at $0{ }^{\circ} \mathrm{C}$. The resulting suspension was stirred at $60^{\circ} \mathrm{C}$ overnight, cooled to room temperature, and treated with water $(50 \mathrm{~mL})$. The resulting precipitate was removed by filtration. The filtrate was diluted with $\mathrm{CH}_{2} \mathrm{Cl}_{2}(150 \mathrm{~mL})$, washed with water $(100 \mathrm{~mL})$, and the organic fraction was concentrated under reduced pressure. The resulting residue was chromatographed $\left(\mathrm{SiO}_{2}\right.$ : hexane-ethyl acetate $\left.=1: 1\right)$ to give $\mathbf{S 9}(1.53 \mathrm{~g}, 93.5 \%)$ as a pale yellow solid. Spectroscopy matched with published data. ${ }^{S 3}$

S10. Reaction of S9 $(1.53 \mathrm{~g}, 4.16 \mathrm{mmol})$, triethylamine $(1.74 \mathrm{~mL}, 12.5 \mathrm{mmol})$, and methanesulfonyl chloride $(0.967 \mathrm{~mL}, 12.5 \mathrm{mmol})$ employing a procedure analogous to that used to synthesize $\mathbf{S} 3$ gave $\mathbf{S} 10$ (2.17 g, 99.7\%) as off-white, needle-like crystals. ${ }^{1} \mathrm{H}$ NMR (500 MHz, $\left.\mathrm{CDCl}_{3}\right): \delta 7.61$ (d, $\left.J=2.4 \mathrm{~Hz}, 1 \mathrm{H}\right), 7.60(\mathrm{~d}, J=2.3 \mathrm{~Hz}, 1 \mathrm{H}), 7.20(\mathrm{dd}, J=8.3,3.2 \mathrm{~Hz}, 2 \mathrm{H}), 6.75$ (dd, $J=8.3,2.4 \mathrm{~Hz}, 2 \mathrm{H}), 4.57-4.54(\mathrm{~m}, 2 \mathrm{H}), 4.43(\mathrm{t}, J=6.1 \mathrm{~Hz}, 2 \mathrm{H}), 4.22-4.18(\mathrm{~m}, 2 \mathrm{H}), 4.05$ $(\mathrm{t}, J=5.8 \mathrm{~Hz}, 2 \mathrm{H}), 3.07(\mathrm{~s}, 3 \mathrm{H}), 2.98(\mathrm{~s}, 3 \mathrm{H}), 2.95-2.90(\mathrm{~m}, 4 \mathrm{H}), 2.84-2.79(\mathrm{~m}, 4 \mathrm{H}), 2.21(\mathrm{p}, J$ $=5.9 \mathrm{~Hz}, 2 \mathrm{H}) .{ }^{13} \mathrm{C}\left\{{ }^{1} \mathrm{H}\right\} \operatorname{NMR}\left(126 \mathrm{MHz}, \mathrm{CDCl}_{3}\right): \delta 156.95,156.40,141.91,141.79,141.74$, $141.33,135.82,135.44,125.99,125.93,114.48,114.33,109.41,68.61,67.16,66.32,63.63$, $37.83,37.27,35.50,35.47,29.99,29.98,29.24$. HRMS (ESI $)$ calcd (found) for $\mathrm{C}_{25} \mathrm{H}_{31} \mathrm{O}_{8} \mathrm{~S}_{2}[+\mathrm{H}]^{+}$: $523.1457(523.1457)$.

$\mathbf{Z}(2,3)$. Reaction of bisphosphine diol (663 mg, $1.20 \mathrm{mmol})$, $\mathbf{S} 10$ (625 mg, $1.20 \mathrm{mmol}$ ), and potassium carbonate $(995 \mathrm{mg}, 7.20 \mathrm{mmol}$ ) employing a procedure analogous to that used to synthesize $Z(2,2)$ gave $Z(2,3)(502 \mathrm{mg}, 47.3 \%)$ as a white solid. ${ }^{1} \mathrm{H}$ NMR $\left(500 \mathrm{MHz}, \mathrm{CD}_{2} \mathrm{Cl}_{2}\right): \delta$ $7.58(\mathrm{~s}, 1 \mathrm{H}), 7.50(\mathrm{~s}, 1 \mathrm{H}), 7.39-7.18(\mathrm{~m}, 24 \mathrm{H}), 6.90(\mathrm{~d}, J=8.3 \mathrm{~Hz}, 1 \mathrm{H}), 6.84-6.70(\mathrm{~m}, 5 \mathrm{H})$, $3.95(\mathrm{t}, J=8.7 \mathrm{~Hz}, 2 \mathrm{H}), 3.85-3.74(\mathrm{~m}, 4 \mathrm{H}), 3.23-3.16(\mathrm{~m}, 1 \mathrm{H}), 3.15-3.06(\mathrm{~m}, 1 \mathrm{H}), 3.00-$ $2.90(\mathrm{~m}, 4 \mathrm{H}), 2.88-2.77(\mathrm{~m}, 4 \mathrm{H}), 1.86-1.76(\mathrm{~m}, 1 \mathrm{H}), 1.76-1.66(\mathrm{~m}, 1 \mathrm{H}) .{ }^{13} \mathrm{C}\left\{{ }^{1} \mathrm{H}\right\} \mathrm{NMR}(126$ $\left.\mathrm{MHz}, \mathrm{CD}_{2} \mathrm{Cl}_{2}\right): \delta 157.86,157.83,157.81,157.78,157.49,157.45,157.41,157.40,157.35$, 
$142.11,141.74,141.32,141.07,139.74,139.68,139.67,139.65,139.64,139.57,139.53,139.40$ 139.36, 139.31, 139.28, 139.27, 139.24, 139.20, 137.45, 137.39, 137.35, 137.32, 137.31, 136.34, $135.31,135.11,135.05,135.00,134.93,134.87,134.82,134.76,133.71,133.65,133.61,133.55$, $133.50,133.44,133.39,133.32,133.27,133.20,133.13,133.08,133.01,128.95,128.86,128.84$, $128.64,128.62,128.45,128.37,128.32,126.49,126.26,126.20,125.91,117.05,113.74,112.61$, $112.28,110.02,107.09,67.71,66.63,64.84,64.29,35.85,35.72,30.28,30.22,30.17 .{ }^{31} \mathrm{P}$ NMR (202 MHz, $\mathrm{CD}_{2} \mathrm{Cl}_{2}$ ): $\delta$-13.87. HRMS (ESI ${ }^{+}$) calcd (found) for $\mathrm{C}_{59} \mathrm{H}_{50} \mathrm{O}_{4} \mathrm{P}_{2}[\mathrm{MH}]^{+}: 885.3179$ (885.3256).

$E(2,3)$. A solution of $Z(2,3)(502 \mathrm{mg}, 0.568 \mathrm{mmol})$ in $\mathrm{CH}_{2} \mathrm{Cl}_{2}(300 \mathrm{~mL})$ was sparged with nitrogen for $20 \mathrm{~min}$ and then irradiated $(\lambda=365 \mathrm{~nm})$ for $1 \mathrm{~h}$ with vigorous stirring to form a photostationary state consisting of a $52: 58$ mixture of $\mathbf{Z}(2,3)$ and $\mathbf{E}(2,3)$. The solvent was evaporated under vacuum and the residue was chromatographed $\left(\mathrm{SiO}_{2}: 10 \%\right.$ ethyl acetate in hexanes) to yield $\mathbf{E}(2,3)(290 \mathrm{mg}, 57.8 \%)$ as a white solid. ${ }^{1} \mathrm{H} \mathrm{NMR}\left(500 \mathrm{MHz}, \mathrm{CD}_{2} \mathrm{Cl}_{2}\right): \delta 7.47-7.34(\mathrm{~m}, 6 \mathrm{H})$, $7.32-7.25(\mathrm{~m}, 5 \mathrm{H}), 7.22(\mathrm{td}, J=8.0,6.6 \mathrm{~Hz}, 2 \mathrm{H}), 7.15-7.05(\mathrm{~m}, 6 \mathrm{H}), 6.99(\mathrm{td}, J=7.6,1.7 \mathrm{~Hz}$, 2H), $6.88-6.76(\mathrm{~m}, 7 \mathrm{H}), 6.64(\mathrm{dd}, J=8.2,2.3 \mathrm{~Hz}, 1 \mathrm{H}), 6.53(\mathrm{tt}, J=6.7,1.4 \mathrm{~Hz}, 2 \mathrm{H}), 6.05(\mathrm{~d}, J=$ $8.3 \mathrm{~Hz}, 1 \mathrm{H}), 3.98-3.89(\mathrm{~m}, 3 \mathrm{H}), 3.77-3.69(\mathrm{~m}, 1 \mathrm{H}), 3.58-3.48(\mathrm{~m}, 2 \mathrm{H}), 3.06-2.65(\mathrm{~m}, 8 \mathrm{H})$, $2.39-2.28(\mathrm{~m}, 1 \mathrm{H}), 1.69(\mathrm{ddd}, J=13.5,9.2,4.5 \mathrm{~Hz}, 1 \mathrm{H}), 1.11(\mathrm{tdd}, J=14.0,9.2,4.4 \mathrm{~Hz}, 1 \mathrm{H})$, $0.44-0.33(\mathrm{~m}, 1 \mathrm{H}) .{ }^{13} \mathrm{C}\left\{{ }^{1} \mathrm{H}\right\}$ NMR: $\left(126 \mathrm{MHz}, \mathrm{CD}_{2} \mathrm{Cl}_{2}\right): \delta 157.59,157.51,157.36,156.97,156.88$, $155.30,146.15,145.05,144.05,140.72,140.42,140.30,139.53,139.48,139.30,139.21,138.68$ 138.56, 138.50, 138.30, 138.19, 135.66, 135.08, 134.10, 134.07, 133.94, 133.91, 133.33, 133.18, $132.87,132.72,128.99,128.94,128.92,128.78,128.73,128.65,128.62,128.57,128.53,128.48$, $128.34,128.31,128.27,128.19,127.74,127.59,126.77,125.70,120.74,120.14,118.42,113.04$, $111.90,108.73,70.78,68.14,67.97,65.44,35.01,32.14,31.65,28.73 .{ }^{31} \mathrm{P}$ NMR $(202 \mathrm{MHz}$, $\mathrm{CD}_{2} \mathrm{Cl}_{2}$ ): $\delta-14.61,-14.99$. HRMS (ESI ${ }^{+}$) calcd (found) for $\mathrm{C}_{59} \mathrm{H}_{50} \mathrm{O}_{4} \mathrm{P}_{2}[\mathrm{MH}]^{+}: 885.3179$ (885.3267). 
Ligand Indane-Biphep was synthesized from 5-indanol employing the sequence depicted in Scheme S3.

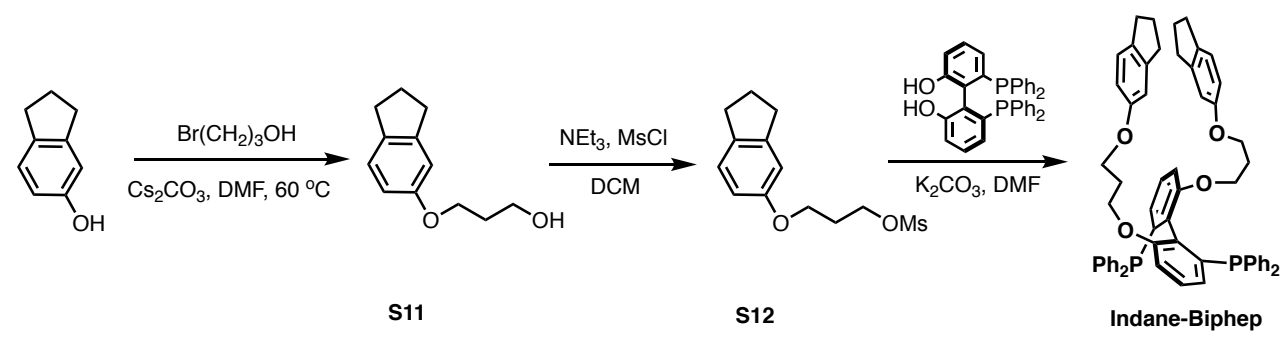

Scheme S3. Synthesis of ligand Indane-Biphep.

3-((2,3-dihydro-1H-inden-5-yl)oxy)propan-1-ol (S11). Cesium carbonate (12.00 g, 36.8 $\mathrm{mmol})$ was added to a solution of 5 -indanol $(2.00 \mathrm{~g}, 14.9 \mathrm{mmol})$ in dimethylformamide $(20 \mathrm{~mL})$ at room temperature. The resulting suspension was heated at $80^{\circ} \mathrm{C}$, and 3-bromo-1-propanol $(2.00$ $\mathrm{mL}, 22.1 \mathrm{mmol}$ ) was added dropwise. The reaction mixture was heated at reflux overnight, cooled to room temperature, and solvent was evaporated under reduced pressure. The resulting orange slurry was dissolved in $\mathrm{CH}_{2} \mathrm{Cl}_{2}(100 \mathrm{~mL})$ and the resulting solution was washed with brine (100 $\mathrm{mL})$, dried $\left(\mathrm{Na}_{2} \mathrm{SO}_{4}\right)$, and concentrated under vacuum. The resulting residue was chromatographed $\left(\mathrm{SiO}_{2}\right.$ : hexane-ethyl acetate $\left.=7: 3\right)$ to give $\mathbf{S 1 1}(2.8 \mathrm{~g}, 98 \%)$ as a colorless liquid. ${ }^{1} \mathrm{H}$ NMR $\left(400 \mathrm{MHz}, \mathrm{CDCl}_{3}\right): \delta 7.08(\mathrm{~d}, J=8.2 \mathrm{~Hz}, 1 \mathrm{H}), 6.77(\mathrm{~s}, 1 \mathrm{H}), 6.67$ (dd, $J=8.1,2.4$ $\mathrm{Hz}, 1 \mathrm{H}), 4.12-4.05(\mathrm{~m}, 2 \mathrm{H}), 3.83(\mathrm{dt}, J=8.0,4.9 \mathrm{~Hz}, 2 \mathrm{H}), 2.93(\mathrm{~s}, 1 \mathrm{H}), 2.82(\mathrm{dd}, J=14.6,7.3$ $\mathrm{Hz}, 4 \mathrm{H}), 2.08-1.98(\mathrm{~m}, 4 \mathrm{H}) .{ }^{13} \mathrm{C}\left\{{ }^{1} \mathrm{H}\right\} \operatorname{NMR}\left(101 \mathrm{MHz}, \mathrm{CDCl}_{3}\right): \delta 124.70,112.51,110.62,66.26$, $60.77,33.12,32.04,31.94,25.79$. HRMS $\left(E S{ }^{+}\right)$calcd (found) for $\mathrm{C}_{12} \mathrm{H}_{16} \mathrm{O}_{2}\left(\mathrm{MH}^{+}\right): 193.1223$ (193.1228).

S12. Reaction of $\mathbf{S} 11(2.8 \mathrm{~g}, 14.6 \mathrm{mmol})$, triethylamine $(3.1 \mathrm{~mL}, 22.5 \mathrm{mmol})$, and methanesulfonyl chloride $(1.75 \mathrm{~mL}, 22.5 \mathrm{mmol})$ employing a procedure analogous to that used to synthesize S3 gave $\mathbf{S 1 2}(3.4 \mathrm{~g}, 86 \%)$ as a white solid. ${ }^{1} \mathrm{H} \mathrm{NMR}\left(400 \mathrm{MHz}, \mathrm{CDCl}_{3}\right): \delta 7.09(\mathrm{~d}, J=$ $8.1 \mathrm{~Hz}, 1 \mathrm{H}), 6.76(\mathrm{~s}, 1 \mathrm{H}), 6.66(\mathrm{dd}, J=8.2,2.4 \mathrm{~Hz}, 1 \mathrm{H}), 4.41(\mathrm{~d}, J=6.2 \mathrm{~Hz}, 2 \mathrm{H}), 4.04(\mathrm{~d}, J=5.8$ 
Hz, 2H), 2.97 (s, 3H), $2.83(\mathrm{dt}, J=14.9,7.1 \mathrm{~Hz}, 4 \mathrm{H}), 2.19(\mathrm{pd}, J=6.1,1.4 \mathrm{~Hz}, 2 \mathrm{H}), 2.10-1.99$ $(\mathrm{m}, 2 \mathrm{H}) .{ }^{13} \mathrm{C}\left\{{ }^{1} \mathrm{H}\right\}$ NMR $\left(126 \mathrm{MHz}, \mathrm{CDCl}_{3}\right): \delta 157.36,145.79,136.63,124.78,112.49,110.58$, $66.88,63.39,37.18,33.12,31.94,29.17,25.79$. HRMS $\left(\mathrm{ESI}^{+}\right)$calcd (found) for $\mathrm{C}_{13} \mathrm{H}_{18} \mathrm{O}_{4} \mathrm{~S}[+\mathrm{H}]^{+}$: 271.0999 (271.1004).

Indane-Biphep. Reaction of bisphosphine diol (510 mg, $0.92 \mathrm{mmol}), \mathbf{S 1 2}(547 \mathrm{mg}, 2.03$ $\mathrm{mmol})$, and potassium carbonate $(760 \mathrm{mg}, 5.51 \mathrm{mmol})$ employing a procedure analogous to that used to synthesize $\mathbf{Z}(\mathbf{2 , 2})$ gave Indane-Biphep (328 mg, 39.5\%) as a white solid. ${ }^{1} \mathrm{H}$ NMR (400 $\left.\mathrm{MHz}, \mathrm{CDCl}_{3}\right): \delta \quad \delta 7.27-7.15(\mathrm{~m}, 22 \mathrm{H}), 7.02(\mathrm{~d}, J=8.2 \mathrm{~Hz}, 2 \mathrm{H}), 6.73(\mathrm{~d}, J=7.6 \mathrm{~Hz}, 2 \mathrm{H}), 6.64$ (d, J = 8.2 Hz, 2H), 6.55 (s, 2H), 6.45 (dd, J = 8.2, $2.5 \mathrm{~Hz}, 2 \mathrm{H}$ ), $3.63-3.51$ (m, 4H), 3.47 (dt, $J=$ 9.4, $6.8 \mathrm{~Hz}, 2 \mathrm{H}$ ), 3.37 (dt, J = 9.1, $5.4 \mathrm{~Hz}, 2 \mathrm{H}), 2.80$ (q, J = 7.4 Hz, 8H), $2.05-2.00(\mathrm{~m}, 4 \mathrm{H}), 1.66$ - $1.50(\mathrm{~m}, 4 \mathrm{H}) .{ }^{13} \mathrm{C}\left\{{ }^{1} \mathrm{H}\right\}$ NMR $\left(101 \mathrm{MHz}, \mathrm{CDCl}_{3}\right): \delta$ 157.60, 134.39, 133.17, 128.46, 128.12, $128.02,127.72,125.84,124.54,112.29,111.15,110.42,64.30,63.86,33.12,31.91,28.85,25.79$. ${ }^{31} \mathrm{P} \mathrm{NMR}\left(162 \mathrm{MHz}, \mathrm{CDCl}_{3}\right): \delta$-13.87. HRMS (ESI ${ }^{+}$) calcd (found) for $\mathrm{C}_{60} \mathrm{H}_{56} \mathrm{O}_{4} \mathrm{P}_{2}[\mathrm{MH}]^{+}: 903.3727$ (903.3747).

\section{Palladium dichloride complexes}

General procedure. An equimolar solution of (COD)PdCl $2(0.06 \mathrm{M})$ and phosphine ligand in $\mathrm{CH}_{2} \mathrm{Cl}_{2}(1-2 \mathrm{~mL})$ was stirred at room temperature for $15 \mathrm{~min}$. The solvent was evaporated under reduced pressure and the resulting residue was recrystallized from $\mathrm{CH}_{2} \mathrm{Cl}_{2}$-diethyl ether to give the desired (P-P)PdCl 2 complexes.

$[\mathbf{Z}(\mathbf{2}, \mathbf{2})] \mathbf{P d C l}_{2}$. Reaction of (COD)PdCl $2(16 \mathrm{mg}, 0.056 \mathrm{mmol})$ and $\mathbf{Z}(\mathbf{2}, \mathbf{2})(51 \mathrm{mg}, 0.059$ mmol) gave $[\mathrm{Z}(2,2)] \mathrm{PdCl}_{2}(50 \mathrm{mg}, 85 \%)$ as yellow solid. ${ }^{1} \mathrm{H} \mathrm{NMR}\left(500 \mathrm{MHz}, \mathrm{CD}_{2} \mathrm{Cl}_{2}\right): \delta 7.91$ (s, 4H), $7.71(\mathrm{dd}, J=12.1,7.7 \mathrm{~Hz}, 4 \mathrm{H}), 7.54(\mathrm{~s}, 2 \mathrm{H}), 7.51-7.47(\mathrm{~m}, 4 \mathrm{H}), 7.45-7.40(\mathrm{~m}, 4 \mathrm{H}), 7.36$ (t, $J=7.7 \mathrm{~Hz}, 4 \mathrm{H}), 7.16(\mathrm{~d}, J=8.2 \mathrm{~Hz}, 2 \mathrm{H}), 6.88(\mathrm{ddd}, J=9.9,5.9,2.3 \mathrm{~Hz}, 2 \mathrm{H}), 6.72(\mathrm{~d}, J=8.5$ 
$\mathrm{Hz}, 2 \mathrm{H}), 6.66(\mathrm{dd}, J=11.4,8.1 \mathrm{~Hz}, 2 \mathrm{H}), 6.50(\mathrm{~d}, J=8.4 \mathrm{~Hz}, 2 \mathrm{H}), 4.08(\mathrm{~d}, J=4.9 \mathrm{~Hz}, 6 \mathrm{H}), 4.04-$ $3.97(\mathrm{~m}, 2 \mathrm{H}), 2.98-2.75(\mathrm{~m}, 8 \mathrm{H}) .{ }^{13} \mathrm{C}\left\{{ }^{1} \mathrm{H}\right\} \mathrm{NMR}\left(126 \mathrm{MHz}, \mathrm{CD}_{2} \mathrm{Cl}_{2}\right): \delta$ 157.71, 142.54, 142.40, $136.55,136.18,136.10,132.80,131.85,130.63,130.53,129.41,129.32,128.57,128.47,126.87$, $126.62,126.55,124.87,115.75,114.62,109.73,67.41,67.19,36.11,30.77 .{ }^{31} \mathrm{P}$ NMR $(202 \mathrm{MHz}$, $\mathrm{CD}_{2} \mathrm{Cl}_{2}$ ): $\delta$ 27.16. HRMS (ESI ${ }^{+}$) calcd (found) for $\mathrm{C}_{58} \mathrm{H}_{48} \mathrm{Cl}_{2} \mathrm{O}_{4} \mathrm{P}_{2} \mathrm{Pd}[\mathrm{M}-\mathrm{Cl}]^{+}: 1011.1746$ (1011.1750).

$[\mathbf{Z}(2,3)] \mathbf{P d C l}_{2}$. Reaction of (COD)PdCl $2(12 \mathrm{mg}, 0.042 \mathrm{mmol})$ and $\mathbf{Z}(2,3)(37 \mathrm{mg}, 0.042$ mmol) gave $[Z(2,3)] \mathrm{PdCl}_{2}(31 \mathrm{mg}, 70 \%)$ as yellow solid. ${ }^{1} \mathrm{H} \mathrm{NMR}\left(500 \mathrm{MHz}, \mathrm{CD}_{2} \mathrm{Cl}_{2}\right): \delta 7.92(\mathrm{~d}, J$ $=16.4 \mathrm{~Hz}, 4 \mathrm{H}), 7.69(\mathrm{ddd}, J=19.4,12.1,7.8 \mathrm{~Hz}, 4 \mathrm{H}), 7.54(\mathrm{~s}, 1 \mathrm{H}), 7.52-7.37(\mathrm{~m}, 9 \mathrm{H}), 7.38-$ $7.25(\mathrm{~m}, 4 \mathrm{H}), 7.18(\mathrm{dd}, J=11.0,8.4 \mathrm{~Hz}, 2 \mathrm{H}), 6.92(\mathrm{td}, J=8.3,2.6 \mathrm{~Hz}, 1 \mathrm{H}), 6.80-6.61(\mathrm{~m}, 4 \mathrm{H})$, $6.59-6.45(\mathrm{~m}, 3 \mathrm{H}), 3.98(\mathrm{~m}, 8 \mathrm{H}), 2.85(\mathrm{dt}, J=50.7,6.5 \mathrm{~Hz}, 8 \mathrm{H}), 1.99(\mathrm{ddq}, J=27.5,14.1,6.7$ $\mathrm{Hz}, 2 \mathrm{H}) .{ }^{13} \mathrm{C}\left\{{ }^{1} \mathrm{H}\right\} \mathrm{NMR}\left(126 \mathrm{MHz}, \mathrm{CD}_{2} \mathrm{Cl}_{2}\right): \delta 157.76,157.67,156.79,156.68,156.66,156.58$, $141.59,141.48,141.37,135.60,135.49,135.37,135.15,135.07,135.01,134.93,131.73,131.69$ $130.80,130.72,130.32,130.07,129.82,129.50,129.45,129.40,129.36,128.85,128.32,128.24$ $128.17,127.49,127.40,125.89,125.84,125.52,125.48,125.42,123.86,123.42,115.46,115.32$ $114.47,113.45,109.38,108.97,67.40,66.40,65.67,65.35,64.64,35.23,35.17,29.80,29.78$, 29.63, 15.09. ${ }^{31} \mathrm{P}$ NMR (202 $\left.\mathrm{MHz}, \mathrm{CD}_{2} \mathrm{Cl}_{2}\right): \delta 27.33,27.16 .{ }^{S 5} \mathrm{HRMS}\left(\mathrm{ESI}^{+}\right)$calcd for $\mathrm{C}_{59} \mathrm{H}_{50} \mathrm{Cl}_{2} \mathrm{O}_{4} \mathrm{P}_{2} \mathrm{Pd}[\mathrm{M}-\mathrm{Cl}]^{+}:$1025.1908, found: 1025.1910.

$[\mathbf{Z}(3,3)] \mathrm{PdCl}_{2}$. Reaction of (COD)PdCl $2(32 \mathrm{mg}, 0.112 \mathrm{mmol})$ and $\mathbf{Z}(3,3)(102 \mathrm{mg}, 0.114$ mmol) gave $[\mathrm{Z}(3,3)] \mathrm{PdCl}_{2}(91 \mathrm{mg}, 78 \%)$ as yellow solid. ${ }^{1} \mathrm{H} \mathrm{NMR}\left(500 \mathrm{MHz}, \mathrm{CD}_{2} \mathrm{Cl}_{2}\right): \delta 7.91(\mathrm{~s}$, 4H), $7.75-7.62(\mathrm{~m}, 4 \mathrm{H}), 7.58(\mathrm{~d}, J=2.6 \mathrm{~Hz}, 2 \mathrm{H}), 7.50(\mathrm{td}, J=7.2,1.7 \mathrm{~Hz}, 2 \mathrm{H}), 7.43$ (qd, $J=7.6$, $2.0 \mathrm{~Hz}, 6 \mathrm{H}), 7.26$ (td, $J=7.9,2.4 \mathrm{~Hz}, 4 \mathrm{H}), 7.14(\mathrm{~d}, J=8.1 \mathrm{~Hz}, 2 \mathrm{H}), 6.92(\mathrm{td}, J=8.2,2.8 \mathrm{~Hz}, 2 \mathrm{H})$, 6.61 (ddd, $J=19.3,8.1,2.4 \mathrm{~Hz}, 4 \mathrm{H}), 6.39$ (d, $J=8.3 \mathrm{~Hz}, 2 \mathrm{H}), 3.80(\mathrm{dp}, J=7.4,3.6,2.1 \mathrm{~Hz}, 2 \mathrm{H}$ ), $3.73-3.62(\mathrm{~m}, 2 \mathrm{H}), 3.57(\mathrm{dd}, J=9.6,4.3 \mathrm{~Hz}, 4 \mathrm{H}), 2.98-2.71(\mathrm{~m}, 8 \mathrm{H}), 1.98-1.76(\mathrm{~m}, 4 \mathrm{H})$. ${ }^{3} \mathrm{C}\left\{{ }^{1} \mathrm{H}\right\}$ NMR $\left(126 \mathrm{MHz}, \mathrm{CD}_{2} \mathrm{Cl}_{2}\right): \delta 157.07,156.98,156.42,141.95,141.08,136.00,135.77$, 
$135.37,135.29,132.25,131.20,130.75,130.25,129.84,129.74,129.44,128.48,128.39,127.96$ $127.87,127.57,127.45,126.17,125.51,125.44,124.16,123.72,114.26,113.12,110.19,64.47$ 63.89, 35.18, 30.22, 28.78. ${ }^{31} \mathrm{P}$ NMR $\left(202 \mathrm{MHz}, \mathrm{CD}_{2} \mathrm{Cl}_{2}\right): \delta$ 27.04. HRMS $\left(\mathrm{ESI}^{+}\right)$calc'd for $\mathrm{C}_{60} \mathrm{H}_{52} \mathrm{Cl}_{2} \mathrm{O}_{4} \mathrm{P}_{2} \mathrm{Pd}[\mathrm{M}-\mathrm{Cl}]^{+}:$1039.2064, found: 1039.2051.

$[E(2,3)] \mathrm{PdCl}_{2}$. Reaction of $(\mathrm{COD}) \mathrm{PdCl}_{2}(10 \mathrm{mg}, 0.035 \mathrm{mmol})$ and $\mathbf{E}(2,3)(33 \mathrm{mg}, 0.037$ mmol) gave $[\mathrm{E}(2,3)] \mathrm{PdCl}_{2}(27 \mathrm{mg}, 74 \%)$ as yellow solid. ${ }^{1} \mathrm{H} \mathrm{NMR}\left(500 \mathrm{MHz}, \mathrm{CD}_{2} \mathrm{Cl}_{2}\right): \delta 7.77$ (td, $J=12.8,7.4 \mathrm{~Hz}, 4 \mathrm{H}), 7.58(\mathrm{ddd}, J=24.3,12.0,5.8 \mathrm{~Hz}, 4 \mathrm{H}), 7.45-7.16(\mathrm{~m}, 13 \mathrm{H}), 7.15-7.02$ (m, 3H), $6.92-6.69(\mathrm{~m}, 3 \mathrm{H}), 6.68-6.54(\mathrm{~m}, 3 \mathrm{H}), 6.42(\mathrm{t}, J=7.9 \mathrm{~Hz}, 2 \mathrm{H}), 6.15(\mathrm{~d}, J=8.4 \mathrm{~Hz}$, 1H), $5.41(\mathrm{~d}, J=8.4 \mathrm{~Hz}, 1 \mathrm{H}), 4.35-4.13(\mathrm{~m}, 1 \mathrm{H}), 4.02-3.76(\mathrm{~m}, 5 \mathrm{H}), 3.37(\mathrm{q}, J=8.9 \mathrm{~Hz}, 1 \mathrm{H})$, $2.83-2.73(\mathrm{~m}, 2 \mathrm{H}), 2.68(\mathrm{t}, J=6.9 \mathrm{~Hz}, 2 \mathrm{H}), 2.57-2.25(\mathrm{~m}, 1 \mathrm{H}), 2.18-1.97(\mathrm{~m}, 2 \mathrm{H}), 1.88(\mathrm{dq}$, $J=27.3,6.6,5.6 \mathrm{~Hz}, 2 \mathrm{H}) \cdot{ }^{13} \mathrm{C}\left\{{ }^{1} \mathrm{H}\right\} \mathrm{NMR}\left(126 \mathrm{MHz}, \mathrm{CD}_{2} \mathrm{Cl}_{2}\right): \delta 157.81,157.71,156.83,156.68$, $155.24,146.31,145.45,143.70,141.59,141.36,135.60,135.50,135.44,135.39,135.18,135.04$, $134.74,132.10,131.79,130.74,130.12,129.42,128.09,127.42,126.46,125.93,125.82,125.04$, $124.97,123.86,120.07,118.61,117.50,115.51,115.34,114.51,113.89,113.52,113.14,109.44$ $109.03,106.26,101.80,81.50,70.62,68.19,67.43,66.45,65.90,65.40,64.67,63.34,56.33$, 52.06, 36.95, 35.74, 35.20, 34.63, 31.81, 30.74, 29.63, 28.15, 26.56. ${ }^{31} \mathrm{P} \mathrm{NMR}\left(202 \mathrm{MHz}, \mathrm{CD}_{2} \mathrm{Cl}_{2}\right)$ $\delta$ 28.57, 27.37. ${ }^{\mathrm{S} 5} \mathrm{HRMS}\left(\mathrm{ESI}{ }^{+}\right.$) calc'd for $\mathrm{C}_{59} \mathrm{H}_{50} \mathrm{Cl}_{2} \mathrm{O}_{4} \mathrm{P}_{2} \mathrm{Pd}[\mathrm{M}-\mathrm{Cl}]^{+}:$1025.1908, found: 1025.1915 .

$[\mathrm{E}(\mathbf{3}, \mathbf{3})] \mathrm{PdCl}_{2}$. Reaction of $(\mathrm{COD}) \mathrm{PdCl}_{2}(17 \mathrm{mg}, 0.060 \mathrm{mmol})$ and $\mathrm{E}(\mathbf{3}, 3)(53 \mathrm{mg}, 0.059$ mmol) gave $[\mathrm{E}(3,3)] \mathrm{PdCl}_{2}(34 \mathrm{mg}, 56 \%)$ as yellow solid. ${ }^{1} \mathrm{H} \mathrm{NMR}\left(400 \mathrm{MHz}, \mathrm{CDCl}_{3}\right) \delta 7.85(\mathrm{~s}$, 3H), $7.75-7.60(\mathrm{~m}, 4 \mathrm{H}), 7.47-7.31(\mathrm{~m}, 9 \mathrm{H}), 7.23-7.15(\mathrm{~m}, 6 \mathrm{H}), 6.90(\mathrm{~d}, J=2.4 \mathrm{~Hz}, 2 \mathrm{H}), 6.84$ (td, $J=8.2,2.9 \mathrm{~Hz}, 2 \mathrm{H}), 6.69(\mathrm{dd}, J=8.1,2.3 \mathrm{~Hz}, 2 \mathrm{H}), 6.57(\mathrm{dd}, J=11.2,7.9 \mathrm{~Hz}, 2 \mathrm{H}), 6.24(\mathrm{~d}, J$ $=8.4 \mathrm{~Hz}, 2 \mathrm{H}), 4.32-4.16(\mathrm{~m}, 4 \mathrm{H}), 4.10(\mathrm{q}, \mathrm{J}=7.1 \mathrm{~Hz}, 2 \mathrm{H}), 3.30(\mathrm{~d}, \mathrm{~J}=5.6 \mathrm{~Hz}, 2 \mathrm{H}), 3.17-2.73$ (m, 12H). ${ }^{13} \mathrm{C}\left\{{ }^{1} \mathrm{H}\right\} \operatorname{NMR}\left(126 \mathrm{MHz}, \mathrm{CD}_{2} \mathrm{Cl}_{2}\right): \delta 157.60,147.07,143.47,137.15,137.07,136.91$, 133.92, 132.92, 131.41, 130.34, 130.25, 129.70, 129.60, 128.17, 127.47, 120.37, 115.53, 112.85, 
67.98, 67.19, 38.30, 33.94, 29.61. ${ }^{31} \mathrm{P}$ NMR (162 MHz, $\left.\mathrm{CD}_{2} \mathrm{Cl}_{2}\right): \delta 27.84 . \mathrm{HRMS}\left(E S I^{+}\right)$calc'd for $\mathrm{C}_{60} \mathrm{H}_{52} \mathrm{Cl}_{2} \mathrm{O}_{4} \mathrm{P} 2 \mathrm{Pd}[\mathrm{M}-\mathrm{Cl}]^{+}:$1039.2064, found: 1039.2132.

(Indane-Biphep)PdCl 2 . Reaction of (COD)PdCl $(99 \mathrm{mg}, 0.35 \mathrm{mmol})$ and Indane-Biphep (328 mg, $0.36 \mathrm{mmol}$ ) gave (Indane-Biphep) $\mathrm{PdCl}_{2}(341 \mathrm{mg}, 90 \%)$ as yellow solid. ${ }^{1} \mathrm{H}$ NMR (500 $\left.\mathrm{MHz}, \mathrm{CD}_{2} \mathrm{Cl}_{2}\right) \delta 7.74(\mathrm{~s}, 4 \mathrm{H}), 7.50(\mathrm{dd}, J=12.2,7.7 \mathrm{~Hz}, 4 \mathrm{H}), 7.41-7.26(\mathrm{~m}, 8 \mathrm{H}), 7.18(\mathrm{td}, J=$ 7.8, $2.4 \mathrm{~Hz}, 4 \mathrm{H}), 6.90(\mathrm{~d}, J=8.2 \mathrm{~Hz}, 2 \mathrm{H}), 6.83(\mathrm{td}, J=8.2,2.9 \mathrm{~Hz}, 2 \mathrm{H}), 6.49(\mathrm{dd}, J=11.1,8.0$ $\mathrm{Hz}, 2 \mathrm{H}), 6.42(\mathrm{~d}, J=2.3 \mathrm{~Hz}, 2 \mathrm{H}), 6.40-6.32(\mathrm{~m}, 4 \mathrm{H}), 3.63-3.39(\mathrm{~m}, 8 \mathrm{H}), 2.73-2.57(\mathrm{~m}, 8 \mathrm{H})$, $1.96-1.87(\mathrm{~m}, 4 \mathrm{H}), 1.83-1.73(\mathrm{~m}, 4 \mathrm{H}) \cdot{ }^{13} \mathrm{C}\left\{{ }^{1} \mathrm{H}\right\} \operatorname{NMR}\left(126 \mathrm{MHz}, \mathrm{CD}_{2} \mathrm{Cl}_{2}\right): \delta$ 157.38, 145.66, $135.54,134.98,134.90,131.69,130.61,128.17,128.08,127.40,127.31,125.07,124.64,113.01$ 112.13, 109.93, 63.24, 63.14, 33.03, 31.87, 28.95, 25.69. ${ }^{31} \mathrm{P}$ NMR $\left(202 \mathrm{MHz}, \mathrm{CD}_{2} \mathrm{Cl}_{2}\right): \delta 27.27$. HRMS (ESI ${ }^{+}$) calcd (found) for $\mathrm{C}_{60} \mathrm{H}_{56} \mathrm{Cl}_{2} \mathrm{O}_{4} \mathrm{P}_{2} \mathrm{Pd}[\mathrm{M}-\mathrm{Cl}]^{+}: 1043.2372$ (1043.2396).

(Indane-Biphep)PdMeCl. Reaction of (COD)PdMeCl (8.5 mg, $0.032 \mathrm{mmol}$ ) and IndaneBiphep (30 mg, $0.033 \mathrm{mmol}$ ) gave (Indane-Biphep)PdMeCl (28 mg, 83\%) as greenish yellow solid. ${ }^{1} \mathrm{H}$ NMR $\left(400 \mathrm{MHz}, \mathrm{CD}_{2} \mathrm{Cl}_{2}\right) \delta 7.69-7.47(\mathrm{~m}, 8 \mathrm{H}), 7.46-7.31(\mathrm{~m}, 7 \mathrm{H}), 7.29-7.24(\mathrm{~m}, 1 \mathrm{H})$, $7.24-7.10(\mathrm{~m}, 4 \mathrm{H}), 6.98(\mathrm{~d}, J=8.2 \mathrm{~Hz}, 2 \mathrm{H}), 6.85(\mathrm{q}, J=7.6,6.9 \mathrm{~Hz}, 2 \mathrm{H}), 6.66-6.57(\mathrm{~m}, 1 \mathrm{H})$, $6.55-6.40(\mathrm{~m}, 5 \mathrm{H}), 6.27(\mathrm{dd}, J=13.4,8.3 \mathrm{~Hz}, 2 \mathrm{H}), 3.63-3.46(\mathrm{~m}, 8 \mathrm{H}), 2.84-2.64(\mathrm{~m}, 8 \mathrm{H})$, $2.01(\mathrm{q}, J=7.4 \mathrm{~Hz}, 4 \mathrm{H}), 1.82(\mathrm{q}, J=6.1 \mathrm{~Hz}, 4 \mathrm{H}), 1.18-1.12(\mathrm{~m}, 3 \mathrm{H}) .{ }^{13} \mathrm{C}\left\{{ }^{1} \mathrm{H}\right\} \mathrm{NMR}(101 \mathrm{MHz}$, $\left.\mathrm{CD}_{2} \mathrm{Cl}_{2}\right): \quad \delta 145.56,135.58,135.46,135.22,134.52,130.75,130.08,127.73,127.60,127.30$ $124.53,112.13,111.70,109.97,63.40,62.59,32.99,31.82,28.96,25.65 .{ }^{31} \mathrm{P}$ NMR (162 MHz, $\mathrm{CD}_{2} \mathrm{Cl}_{2}$ ): $\delta 37.13$ (d, $\left.J=41.5 \mathrm{~Hz}\right), 12.87$ (d, $\left.J=41.5 \mathrm{~Hz}\right)$. HRMS (ESI ${ }^{+}$) calc'd for $\mathrm{C}_{61} \mathrm{H}_{55} \mathrm{ClO}_{4} \mathrm{P}_{2} \mathrm{Pd}$ $[\mathrm{M}-\mathrm{Cl}]^{+}:$1019.2610, found: 1039.2622.

\section{Generation of (P-P)Pd(0) Complexes.}

A $1 \mathrm{M}$ solution of $\mathrm{NaBH}_{4}\left(19.7 \mu \mathrm{L}, 1.97 \times 10^{-2} \mathrm{mmol}\right)$ in triglyme was added via syringe to an NMR tube containing a solution of $(\mathrm{P}-\mathrm{P}) \mathrm{PdCl}_{2}\left(9.9 \times 10^{-3} \mathrm{mmol}, 16 \mathrm{mM}\right)$ in THF- $d_{8}$ 
$(0.6 \mathrm{~mL})$ at $-78^{\circ} \mathrm{C}$ and the contents of the tube were mixed thoroughly at room temperature quickly while still cold to form a homogeneous solution. Because ( $\mathrm{MeOBiphep}) \mathrm{PdCl}_{2}$ did not fully dissolve at the concentration of $16 \mathrm{mM}$, less concentrated (MeOBiphep) $\mathrm{PdCl}_{2}$ solution (5 mM) was used in this case to ensure homogeneity. The tube was placed in the probe of an NMR spectrometer precooled at $10{ }^{\circ} \mathrm{C}$ and analyzed by ${ }^{31} \mathrm{P}$ NMR spectroscopy. For each $\mathrm{P}-\mathrm{P}$ ligand investigated except indane-biphep, reduction led to complete consumption of (P-P)PdCl 2 with formation of a single (P-P)Pd(0) complex as judged by ${ }^{31} \mathrm{P} N M R$ spectroscopy; the symmetric $Z(2,2), Z(3,3), E(3,3)$, and MeOBiphep complexes displayed a single sharp ${ }^{31} P$ NMR resonance that was shielded relative to the resonances for the corresponding $(\mathrm{P}-\mathrm{P}) \mathrm{PdCl}_{2}$ complex, while the unsymmetric $Z(2,3)$ and $E(2,3)$ complexes displayed two sharp resonances that were shielded relative to the resonances for the corresponding ( $\mathrm{P}-\mathrm{P}) \mathrm{PdCl}_{2}$ complexes. Reduction of (IndaneBiphep) $\mathrm{PdCl}_{2}$ led to complete consumption of (Indane-Biphep) $\mathrm{PdCl}_{2}$ to form a $\sim 2: 1$ mixture of (indane-biphep) $\mathrm{Pd}(0)$ complexes as judged by ${ }^{31} \mathrm{P}$ NMR spectroscopy; the ${ }^{31} \mathrm{P}$ NMR spectrum displayed a $\sim 2: 1$ ratio of sharp resonances at $\delta 19.79$ and 18.85 which were shielded relative to the resonance for (indane-Biphep) $\mathrm{PdCl}_{2}$ (Figure S1).

\begin{tabular}{llllllllllllllllllllllll}
\hline 23.5 & 23.0 & 22.5 & 22.0 & 21.5 & 21.0 & 20.5 & 20.0 & 19.5 & 19.0 & 18.5 & 18.0 & 17.5 & 17.0 & 16.5 & 16.0 & 15.5 & 15.0 & 14.5 & 14.0
\end{tabular} 


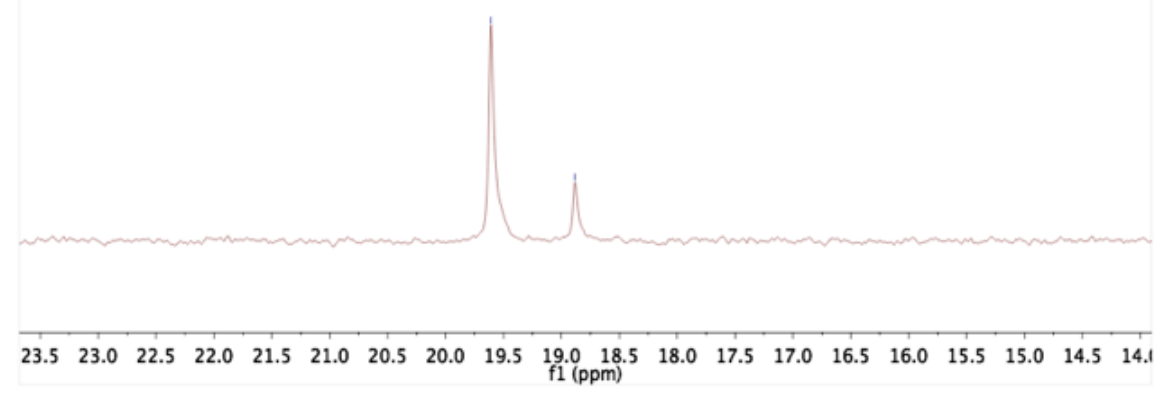

Figure S1. ${ }^{31} \mathrm{P}$ NMR spectra of the (Indane-Biphep)Pd(0) complexes generated from reaction of (Indane-Biphep) $\mathrm{PdCl}_{2}(16 \mathrm{mM})$ with $\mathrm{NaBH}_{4}$ (2 equiv) in THF- $d_{8}$ at $10{ }^{\circ} \mathrm{C}$ (top) and from reaction (Indane-Biphep)PdMeCl with $\mathrm{NaBH}_{4}$ (1 equiv) in $\mathrm{THF}-d_{8}$ at $10^{\circ} \mathrm{C}$.

Analysis of ${ }^{31} \mathrm{P}$ NMR resonances for $(\mathrm{P}-\mathrm{P}) \mathrm{Pd}(0)$ complexes relative to the corresponding (P-P)PdCl $\mathrm{Pd}_{2}$ complexes revealed that for all ligands except $Z(3,3)$ and indane-biphep, the ${ }^{31} \mathrm{P} N M R$ resonances of the $(\mathrm{P}-\mathrm{P}) \mathrm{Pd}(0)$ complexes were shielded by $\Delta \delta=2.2-3.1$ relative to the corresponding (P-P)PdCl 2 complexes (Table S1). In comparison, the ${ }^{31} \mathrm{P}$ NMR resonance of $[\mathrm{Z}(3,3)] \mathrm{Pd}(0)$ was shielded by $\Delta \delta=4.0$ relative to $[\mathrm{Z}(3,3)] \mathrm{PdCl}_{2}$ (Table S1). The ${ }^{31} \mathrm{P}$ NMR resonance of the major (indane-biphep) $\operatorname{Pd}(0)$ complex was shielded by $\Delta \delta=3.0$ relative to (indane-biphep) $\mathrm{PdCl}_{2}$ whereas the minor (indane-biphep) $\mathrm{Pd}(0)$ complex was shielded by $\Delta \delta=3.9$ relative to (indane-biphep) $\mathrm{PdCl}_{2}$ (Table S1). 
Table S1. ${ }^{31} \mathrm{P}$ NMR chemical shifts and ${ }^{2} J_{P P}$ for $(\mathrm{P}-\mathrm{P}) \mathrm{PdCl} \mathrm{P}_{2},(\mathrm{P}-\mathrm{P}) \mathrm{Pd}(0)$, and $(\mathrm{P}-\mathrm{P}) \mathrm{Pd}(\mathrm{Ph}) \mathrm{Br}(16$ $\mathrm{mM})$ in $\mathrm{THF}-d_{8}$ at $10^{\circ} \mathrm{C}$.

\begin{tabular}{|c|c|c|c|c|}
\hline P-P & $(\mathrm{P}-\mathrm{P}) \mathrm{Pd}(\mathrm{II}) \mathrm{Cl}_{2}(\delta)$ & $(\mathrm{P}-\mathrm{P}) \mathrm{Pd}(0)(\delta)$ & $\Delta \delta^{\mathrm{a}}$ & $(\mathrm{P}-\mathrm{P}) \mathrm{Pd}(\mathrm{Ph}) \mathrm{Br}$ \\
\hline $\mathrm{Z}(2,2)$ & 22.90 & 20.26 & 2.6 & $22.50,6.77(\mathrm{~J}=41.5 \mathrm{~Hz})$ \\
\hline $\mathrm{Z}(2,3)$ & $22.88,22.64$ & $20.27,19.67$ & $2.6,2.9$ & $22.98,6.78(\mathrm{~J}=42 \mathrm{~Hz})$ \\
\hline $\mathrm{Z}(3,3)$ & 22.75 & 18.78 & 4.0 & $22.51,6.78(\mathrm{~J}=42 \mathrm{~Hz})$ \\
\hline MeOBiphep $^{\mathrm{b}}$ & 22.86 & 20.15 & 2.7 & $22.79,6.69(\mathrm{~J}=42 \mathrm{~Hz})$ \\
\hline Indane-Biphep $^{2}$ & 22.75 & $19.79,18.85$ & $3.0,3.9$ & $22.72,6.72(\mathrm{~J}=42 \mathrm{~Hz})$ \\
\hline $\mathrm{E}(2,3)$ & $23.71,22.50$ & $22.38,19.42$ & $1.3,3.1$ & $\begin{array}{c}{[25.01,24.48(1: 1)],[5.56,} \\
6.79(1: 1)],(\mathrm{J}=40.2 \mathrm{~Hz})\end{array}$ \\
\hline $\mathrm{E}(3,3)$ & 23.82 & 21.60 & 2.2 & $23.88,6.36(\mathrm{~J}=40 \mathrm{~Hz})$ \\
\hline
\end{tabular}

$\Delta \delta=\delta(\mathrm{P}-\mathrm{P}) \mathrm{PdCl}_{2}-\delta(\mathrm{P}-\mathrm{P}) \mathrm{Pd}(0) .{ }^{\mathrm{b}} 5 \mathrm{mM}$

Kinetic analysis of oxidative addition to (P-P)Pd(0) complexes. Bromobenzene (20.7 $\mu \mathrm{L}, 0.197 \mathrm{mmol})$ was added via syringe to an NMR tube containing a solution of $(\mathrm{P}-\mathrm{P}) \mathrm{Pd}(0)[\mathrm{P}-\mathrm{P}$ $=Z(2,2), Z(2,3), Z(3,3), E(2,3), E(3,3)]\left(9.9 \times 10^{-3} \mathrm{mmol}, 16 \mathrm{mM}\right)$ in THF- $d_{8}$ at $-78{ }^{\circ} \mathrm{C}$ and the resulting solution was inserted into the probe of an NMR spectrometer precooled at $10{ }^{\circ} \mathrm{C}$ and analyzed periodically by ${ }^{31} \mathrm{P}$ NMR spectroscopy. MeOBiphep was measured at the concentration of $5 \mathrm{mM}\left(3.1 \times 10^{-3} \mathrm{mmol}\right)$. The concentration of $(\mathrm{P}-\mathrm{P}) \mathrm{Pd}(0)$ was determined by integrating the ${ }^{31} \mathrm{P}$ NMR resonance(s) of $(\mathrm{P}-\mathrm{P}) \mathrm{Pd}(0)$ (Table $\mathrm{S} 1$ ) relative to the resonance of $\mathrm{H}_{3} \mathrm{PO}_{4}$ external standard at $\delta 0$. Pseudo first-order rate constants were determined from plots of $\ln [(\mathrm{P}-\mathrm{P}) \mathrm{Pd}(0)]$ versus time, typically over the first 3 half-lives of reaction (Figures S2 - S8; Table S2, entries 118). To establish the first-order dependence of the rate on bromobenzene concentration, pseudo first-order rate constants were determined for the reaction of (MeOBiphep) $\operatorname{Pd}(0)(33 \mathrm{mM})$ with bromobenzene from 0 to $1.2 \mathrm{M}$ in acetone- $d_{6}$ at $5{ }^{\circ} \mathrm{C}$ (Table S2, entries 15-18). A plot of $k_{\text {obs }}$ versus [bromobenzene] was linear with a second-order rate constant of $1.67 \pm 0.40 \mathrm{M}^{-1} \mathrm{~s}^{-1}$ (Figure 
S8), which established the first-order dependence of the rate of oxidation on the concentration of bromobenzene.

Table S2. Pseudo first-order rate constants for the reaction of $(P-P) P d(0)(16 \mathrm{mM})$ with bromobenzene $(0.32 \mathrm{M})$ in THF- $d_{8}$ at $10^{\circ} \mathrm{C}$.

\begin{tabular}{|c|c|c|c|}
\hline Entry & $\mathrm{P}-\mathrm{P}$ & {$[\mathrm{PhBr}](\mathrm{M})$} & $\left(10^{4}\right) k_{\text {obs }}\left(\mathrm{s}^{-1}\right)$ \\
\hline 1 & $\mathrm{Z}(2,2)$ & 0.32 & $8.10 \pm 0.35$ \\
\hline 2 & $\mathrm{Z}(2,2)^{-}$ & 0.32 & $7.57 \pm 0.31$ \\
\hline 3 & $\mathrm{Z}(2,3)$ & 0.32 & $6.95 \pm 0.51$ \\
\hline 4 & $\mathrm{Z}(2,3)$ & 0.32 & $19.32 \pm 0.96$ \\
\hline 5 & $\mathrm{Z}(3,3)$ & 0.32 & $19.05 \pm 1.00$ \\
\hline 6 & $Z(3,3)$ & 0.32 & $5.38 \pm 0.33$ \\
\hline $7^{\mathrm{a}}$ & MeOBiphep & 0.32 & $6.15 \pm 0.64$ \\
\hline $8^{\mathrm{a}}$ & MeOBiphep & 0.32 & $1.38 \pm 0.10$ \\
\hline 11 & E(2,3) & 0.32 & $1.32 \pm 0.07$ \\
\hline 12 & E(2,3) & 0.32 & $1.15 \pm 0.03$ \\
\hline 13 & E(3,3) & 0.32 & $1.13 \pm 0.04$ \\
\hline 14 & E(3,3) & 0.32 & $0.62 \pm 0.05$ \\
\hline $15^{\mathrm{b}}$ & MeOBiphep & 0.61 & $1.29 \pm 0.09$ \\
\hline $16^{\mathrm{b}}$ & MeOBiphep & 0.89 & $1.35 \pm 0.11$ \\
\hline $17^{\mathrm{b}}$ & MeOBiphep & 1.15 & $2.03 \pm 0.18$ \\
\hline $18^{\mathrm{b}}$ & MeOBiphep & $5 \mathrm{mM}$ & \\
\hline
\end{tabular}

${ }^{\mathrm{a}}$ Reactions run with $[(\mathrm{MeOBiphep}) \operatorname{Pd}(0)]_{0}=5 \mathrm{mM}$. ${ }^{\mathrm{b}}$ Reactions run in acetone- $d_{6}$ at $5{ }^{\circ} \mathrm{C}$ with $[(\text { MeOBiphep }) \operatorname{Pd}(0)]_{0}=33 \mathrm{mM}$. 

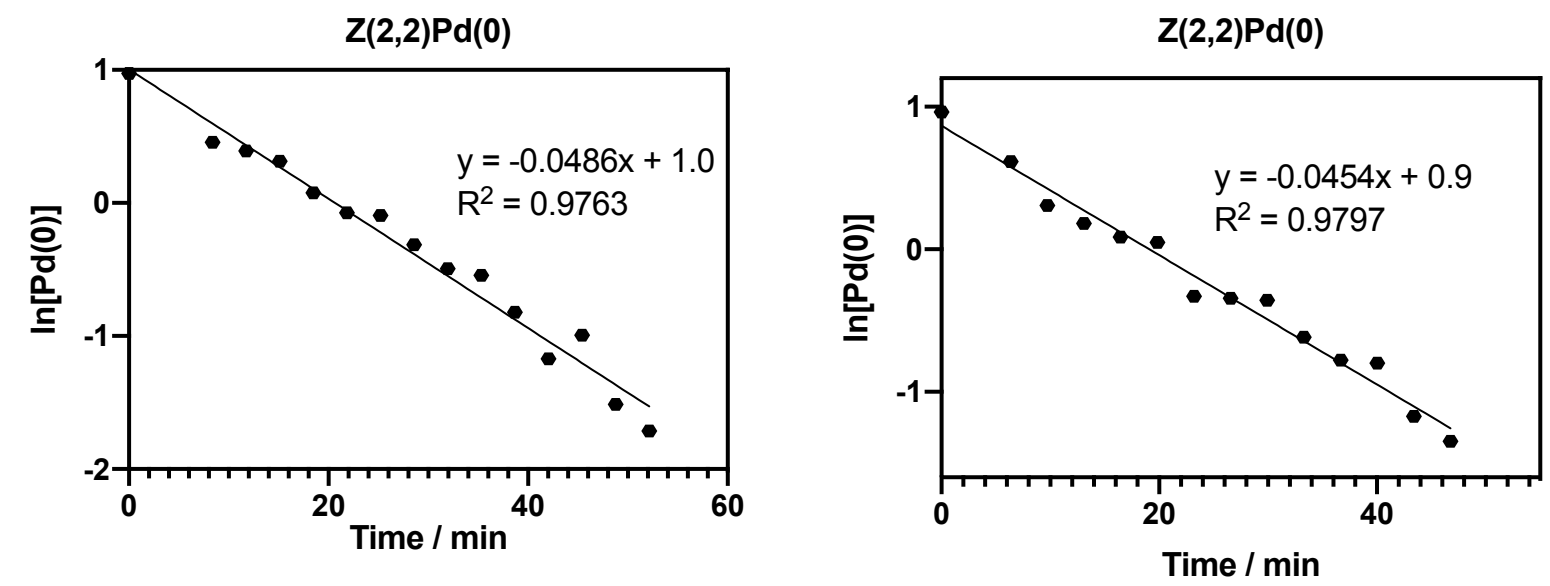

Figure S2. Pseudo first-order plots of the reaction of $[\mathbf{Z}(2,2)] P d(0)(16 \mathrm{mM})$ with bromobenzene $(0.32 \mathrm{M})$ in $\mathrm{THF}-\mathrm{d}_{8}$ at $10^{\circ} \mathrm{C}$.
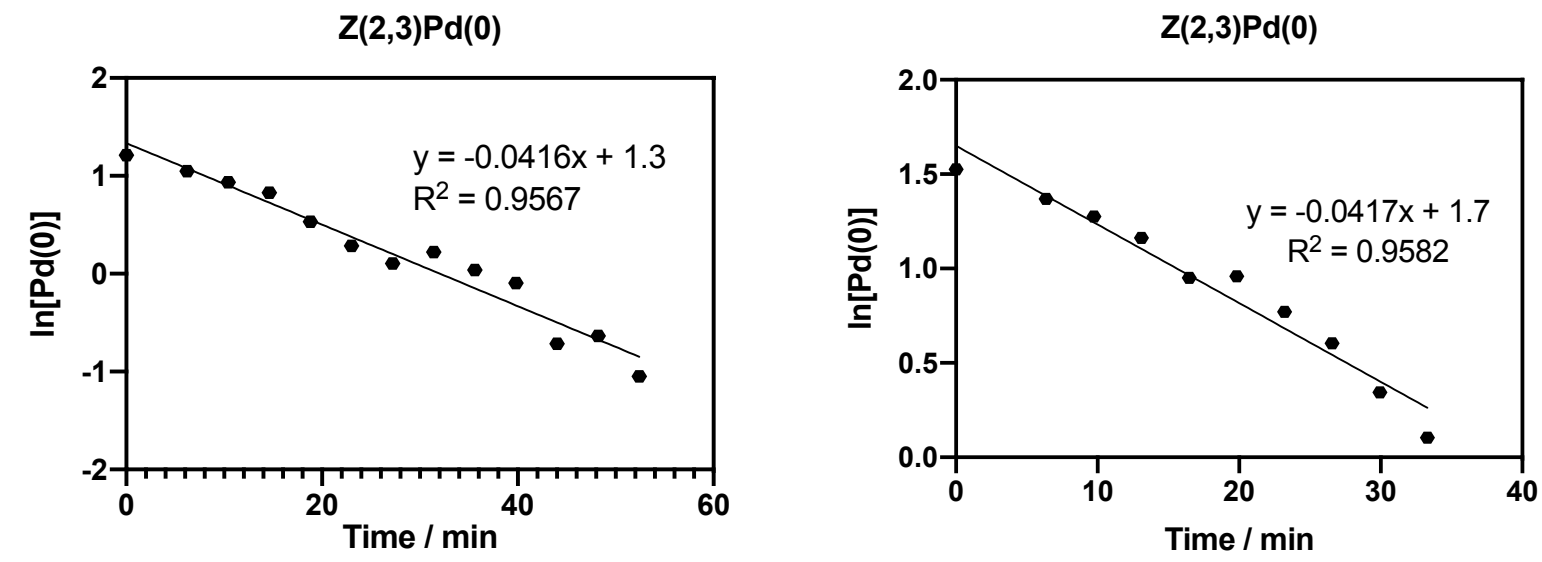

Figure S3. Pseudo first-order plots of the reaction of $[Z(2,3)] P d(0)(16 \mathrm{mM})$ with bromobenzene $(0.32 \mathrm{M})$ in THF- $d_{8}$ at $10^{\circ} \mathrm{C}$. 

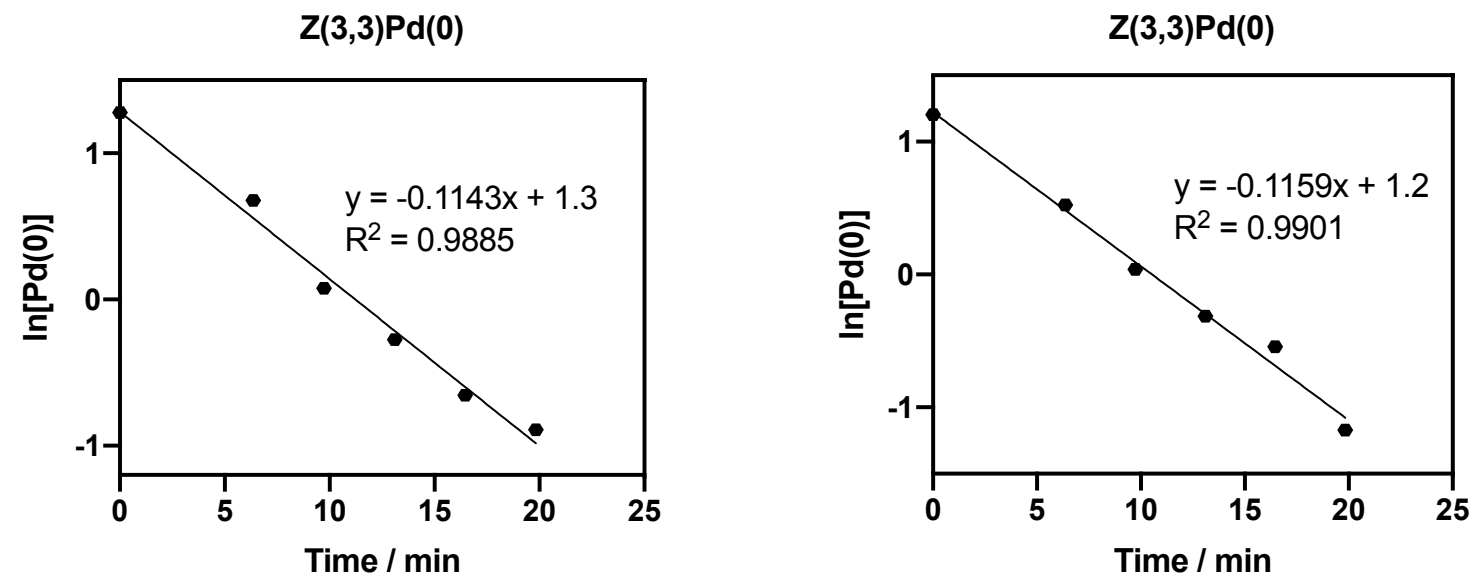

Figure S4. Pseudo first-order plots of the reaction of $[Z(3,3)] P d(0)(16 \mathrm{mM})$ with bromobenzene $(0.32 \mathrm{M})$ in $\mathrm{THF}-d_{8}$ at $10^{\circ} \mathrm{C}$.
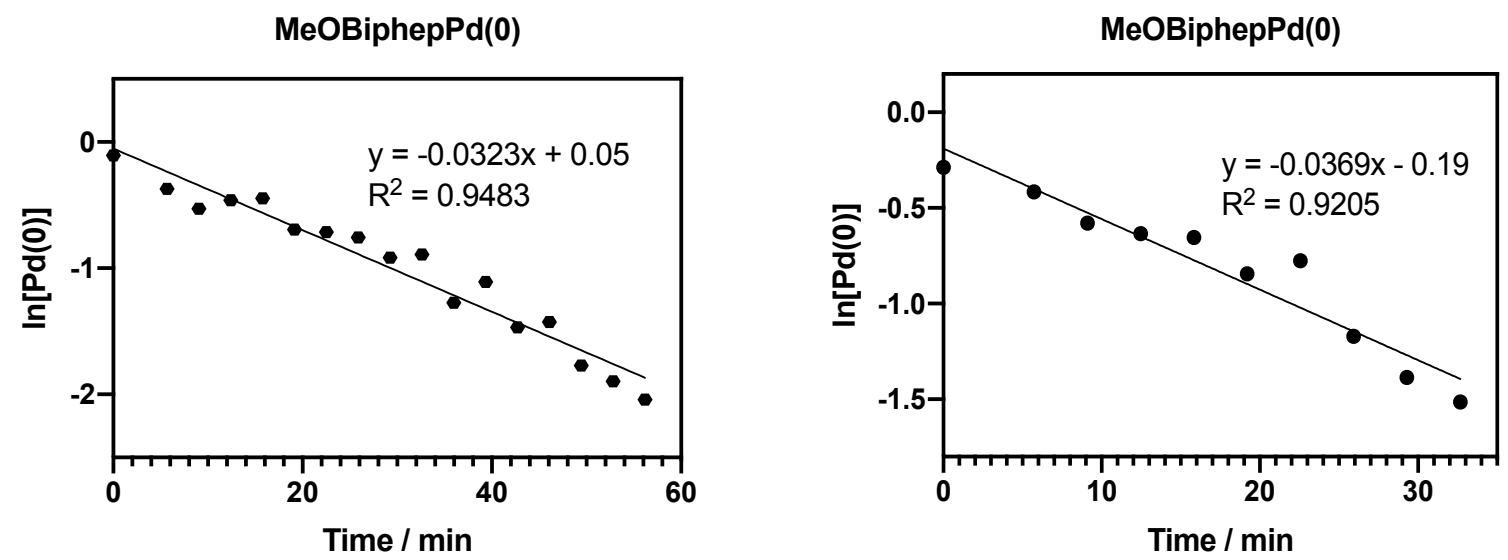

Figure S5. Pseudo first-order plots of the reaction of $[\mathrm{MeOBiphep}] \operatorname{Pd}(0)(5 \mathrm{mM})$ with bromobenzene $(0.32 \mathrm{M})$ in THF- $d_{8}$ at $10^{\circ} \mathrm{C}$. 

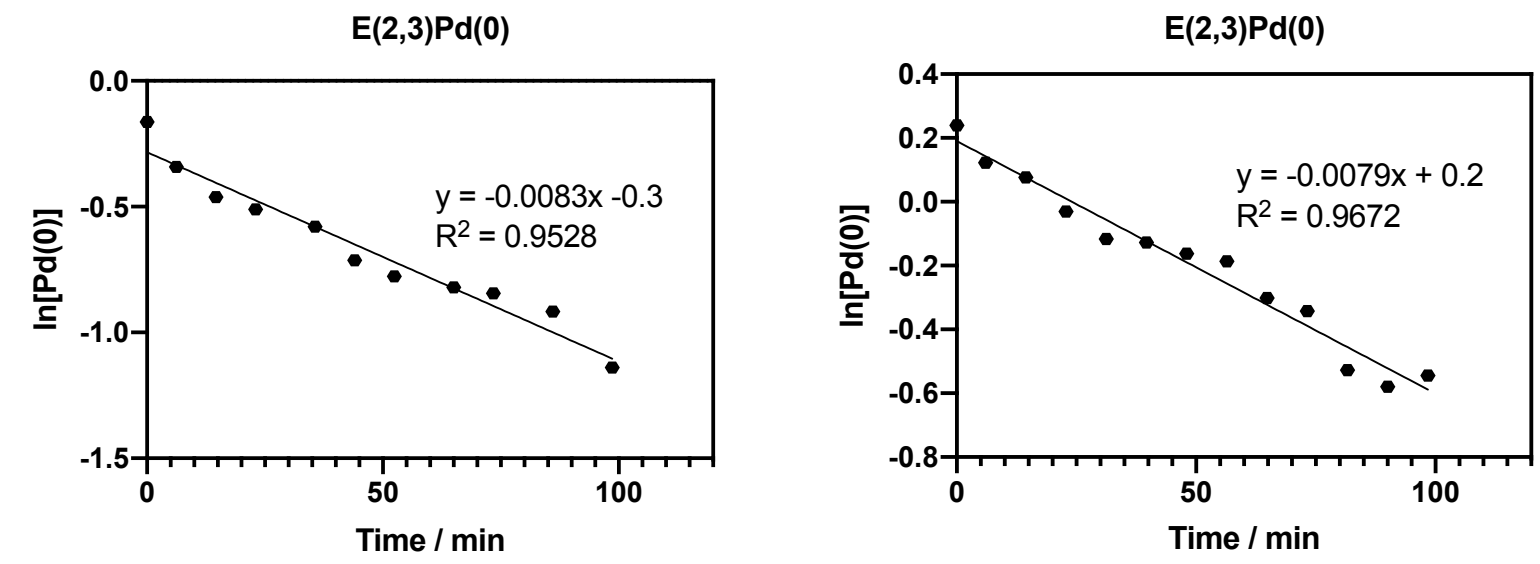

Figure S6. Pseudo first-order plots of the reaction of $[E(2,3)] P d(0)(16 \mathrm{mM})$ with bromobenzene $(0.32 \mathrm{M})$ in THF- $d_{8}$ at $10^{\circ} \mathrm{C}$.
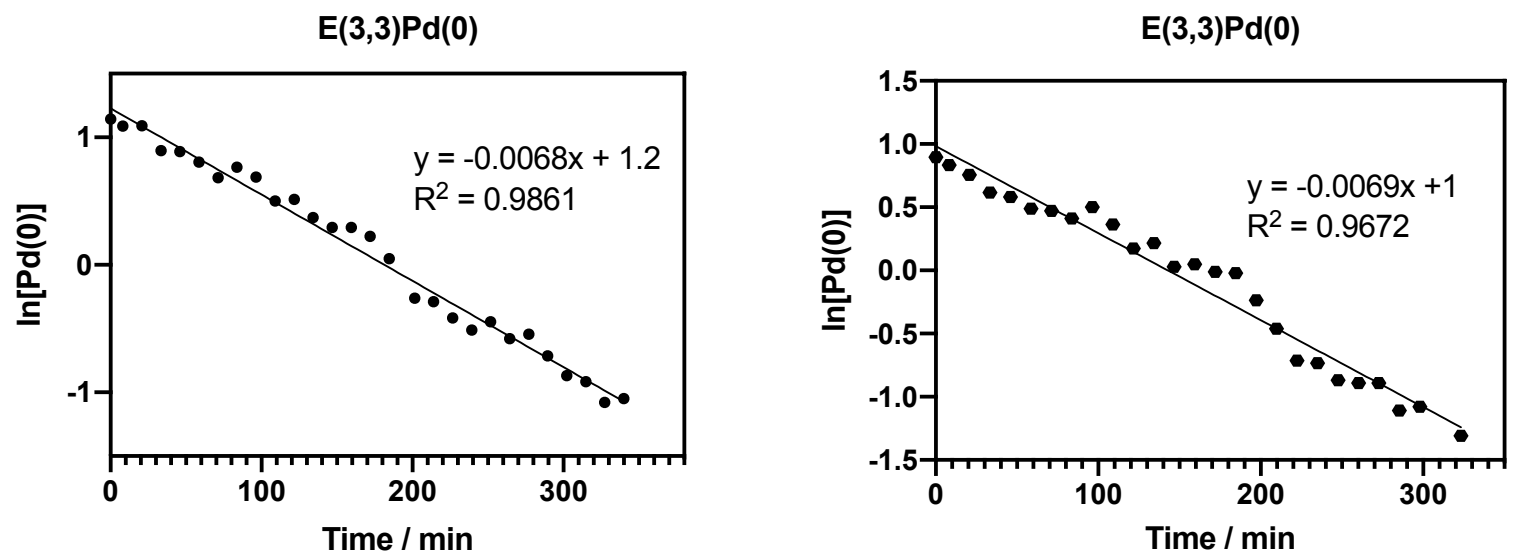

Figure S7. Pseudo first-order plots of the reaction of $[E(3,3)] P d(0)(16 \mathrm{mM})$ with bromobenzene $(0.32 \mathrm{M})$ in $\mathrm{THF}-d_{8}$ at $10^{\circ} \mathrm{C}$. 


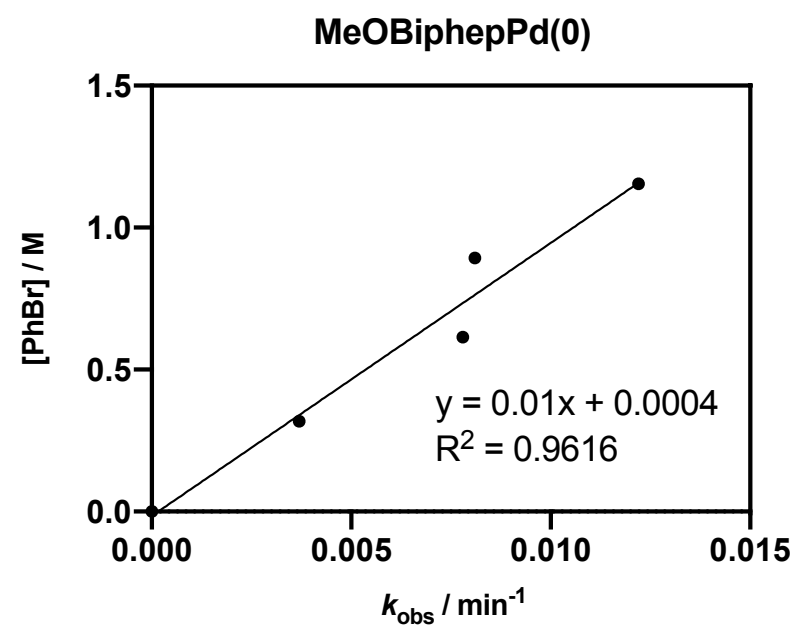

Figure S8. Plot of $k_{\text {obs }}$ versus [bromobenzene] for the reaction of bromobenzene $(0-1.2 \mathrm{M})$ with (MeOBiphep) $\mathrm{Pd}(0)(33 \mathrm{mM})$ in acetone- $d_{6}$ at $5{ }^{\circ} \mathrm{C}$.

Bromobenzene $(20.7 \mu \mathrm{L}, 0.197 \mathrm{mmol})$ was added via syringe to an NMR tube containing a solution comprising a $\sim 2: 1$ mixture of (indane-biphep) $\mathrm{Pd}(0)$ complexes $\left([\mathrm{Pd}]_{\text {tot }}=16 \mathrm{mM}\right)$ in THF$d_{8}$ at $-78{ }^{\circ} \mathrm{C}$ and the resulting solution was inserted into the probe of an NMR spectrometer precooled at $10{ }^{\circ} \mathrm{C}$ and analyzed periodically by ${ }^{31} \mathrm{P}$ NMR spectroscopy. The concentrations of major and minor (indane-biphep) $\mathrm{Pd}(0)$ complexes were determined by integrating the ${ }^{31} \mathrm{P} N \mathrm{NMR}$ resonances at $\delta 19.79,18.85$, respectively relative to the resonance of $\mathrm{H}_{3} \mathrm{PO}_{4}$ external standard at $\delta 0$ (Figure S1). Analysis of concentration versus time plots erevealed the rapid disappearance of the minor (indane-biphep) $\mathrm{Pd}(0)$ complex superimposed on the slower disappearance of the major (indane-biphep) $\mathrm{Pd}(0)$ complex (Figure S9). The corresponding plots of the natural logarithm of concentration of the major and minor (indane-biphep) $\operatorname{Pd}(0)$ complexes versus time provided satisfactory fit with pseudo first-order rate constants of $3.8 \pm 0.1 \times 10^{-4} \mathrm{~s}^{-1}$ and $2.2 \times 10^{-}$ ${ }^{3} \mathrm{~s}^{-1}$, respectively (Figure S9). The former value is approximately $\sim 60 \%$ of the magnitude of the pseudo first-order rate constant obtained for reaction of (MeOBiphep)Pd(0) with bromobenzene, 
while the latter value is not significantly different from the pseudo first-order rate constant obtained for reaction of $[Z(3,3)] P d(0)$ with bromobenzene (Table S2).
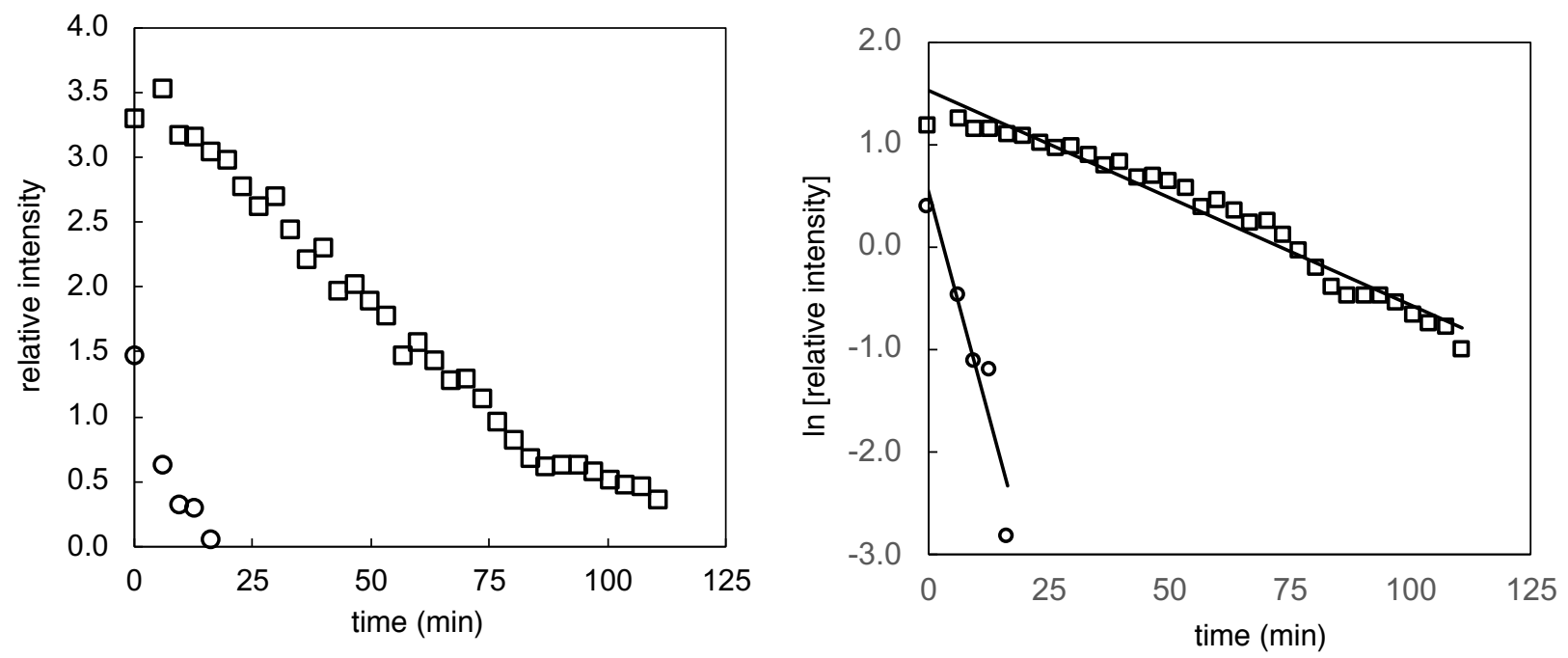

Figure S9. Plots of ${ }^{31} \mathrm{P}$ NMR intensity versus time (left) and natural logarithm of ${ }^{31} \mathrm{P}$ NMR intensity versus time (right) for the reaction of the major $(\square)$ and minor $(O)$ (indane-biphep) $\operatorname{Pd}(0)$ complexes $\left([\mathrm{Pd}]_{\text {tot }}=16 \mathrm{mM}\right)$ with bromobenzene $(0.32 \mathrm{M})$ in THF- $d_{8}$ at $10{ }^{\circ} \mathrm{C}$.

Nature of the $(P-P) P d(0)$ complexes. Our results are consistent with the generation of two discrete forms of $(\mathrm{P}-\mathrm{P}) \mathrm{Pd}(0)$ complexes generated via reduction of $(\mathrm{P}-\mathrm{P}) \mathrm{PdCl}_{2}$ with $\mathrm{NaBH}_{4}$ that are distinguished both by their ${ }^{31} \mathrm{P}$ NMR spectroscopy and reactivity with respect to oxidative addition of bromobenzene. Furthermore, our observations suggest that all P-P ligands employed here with the exceptions of $Z(3,3)$ and indane-biphep generate $(P-P) P d(0)$ complexes of a similar form that differ only in the nature of the P-P ligand and which behave predictably with respect to oxidative addition as a function of applied mechanical force. In comparison, reduction of $[\mathrm{Z}(3,3)] \mathrm{PdCl}_{2}$ generates a $(\mathrm{P}-\mathrm{P}) \mathrm{Pd}(0)$ complex that displays greater shielding in the ${ }^{31} \mathrm{P} N M R$ 
spectrum and enhanced reactivity with respect to oxidative addition. Reduction of (indanebiphep) $\mathrm{PdCl}_{2}$ generates two distinct (indane-biphep) $\mathrm{Pd}(0)$ complexes: a major form that resembles the (P-P)Pd(0) $[P-P=Z(2,2), Z(2,3)$, MeOBiphep, $E(2,3)$, and $E(3,3)]$ complexes both with respect to the ${ }^{31} \mathrm{P}$ NMR spectroscopy and reactivity and a minor form that resembles the $[Z(3,3)] P d(0)$ complex both with respect to the ${ }^{31} \mathrm{P}$ NMR spectroscopy and reactivity.

The composition of the two forms of $(\mathrm{P}-\mathrm{P}) \mathrm{Pd}(0)$ complexes remains unclear. Amatore and Jutand have shown that electrochemical reduction of $\mathrm{L}_{2} \mathrm{PdCl}_{2}\left(\mathrm{~L}=\mathrm{PPh}_{3} ; 17 \mathrm{mM}\right)$ in $\mathrm{THF}$ in the absence of coordinating ligands forms a 0.7/0.6/1.0 mixture of the monochloride anionic complex $\left[\mathrm{L}_{2} \mathrm{PdCl}\right]^{-}$ , the monochloride dimer $\left[\mathrm{L}_{2} \mathrm{PdCl}\right]_{2}{ }^{2-}$ and the dichloride dianion $\left[\mathrm{L}_{2} \mathrm{PdCl}_{2}\right]^{2-}$, all of which equilibrate slowly on the NMR time scale and can be differentiated by ${ }^{31} \mathrm{P}$ NMR spectroscopy. ${ }^{\text {s6 }}$ In comparison, Negishi showed that chemical reduction of $\mathrm{L}_{2} \mathrm{PdCl}_{2}$ with organolithium reagents forms a single species proposed to be $\left[\mathrm{L}_{2} \mathrm{PdCl}\right] \mathrm{Li},\left[\mathrm{L}_{2} \mathrm{PdCl}_{2}\right] \mathrm{Li}_{2}$ or their aggregates, ${ }^{\mathrm{S}, \mathrm{S} 8}$ and Amatore has similarly noted the propensity of anionic palladium( 0 ) chloride complexes to engage in ion pairing. ${ }^{\text {S6 }}$

In the case of the $(\mathrm{P}-\mathrm{P}) \mathrm{Pd}(0)$ complexes generated via $\mathrm{NaBH}_{4}$ reduction of $(\mathrm{P}-\mathrm{P}) \mathrm{PdCl} \mathrm{C}_{2}$ complexes, several scenarios can be excluded. Firstly, reduction of the monochloride precursors $(\mathrm{P}-\mathrm{P}) \mathrm{Pd}(\mathrm{Me}) \mathrm{Cl}\left(\mathrm{P}-\mathrm{P}=\right.$ force probe ligand) with $\mathrm{NaBH}_{4}$ (1 equiv) formed $(\mathrm{P}-\mathrm{P}) \mathrm{Pd}(0)$ complexes that were indistinguishable from those generated from $(\mathrm{P}-\mathrm{P}) \mathrm{PdCl}_{2}$, arguing against $(\mathrm{P}-\mathrm{P}) \mathrm{Pd}(0)$ complexes containing more than one chloride ligand per palladium atom. Similarly, reduction of (indane-biphep) $\mathrm{Pd}(\mathrm{Me}) \mathrm{Cl}$ with $\mathrm{NaBH}_{4}$ formed the same two (indane-biphep) $\mathrm{Pd}(0)$ complexes $(\delta$ 19.79 and 18.85) as formed from (indane-biphep) $\mathrm{PdCl}_{2}$ and in a similar ratio (Figure S1). One possibility, therefore, is that the two forms of (P-P)Pd(0) complexes generated via $\mathrm{NaBH}_{4}$ reduction of (P-P)PdCl 2 complexes correspond to the monochloride anionic complex (P-P)PdCl ${ }^{-}$ and the dichloride dianionic complex $[(\mathrm{P}-\mathrm{P}) \mathrm{PdCl}]_{2}{ }^{2-}$. However, within the framework we can rule out mechanisms involving either (1) rapid and reversible dimerization of a monomeric (P-P)Pd(0) 
complex followed by rate-limiting oxidative addition to the resulting dimeric species or (2) rapid and reversible disproportion of a dimeric $[(\mathrm{P}-\mathrm{P}) \mathrm{Pd}(0)]_{2}$ complex followed by rate-limiting oxidative addition to the monomeric species; the former mechanism would display second-order rate dependence on $[\mathrm{Pd}]$, while the latter would display half-order rate dependence on $[\mathrm{Pd}]$, neither of which were observed. Similarly, the kinetic behavior of the reaction of a $\sim 2: 1$ mixture of (indanebiphep) $\operatorname{Pd}(0)$ complexes with bromobenzene argued strongly against rapid equilibration of the two species followed by rate-determining oxidative addition. For these reasons, our results are consistent with (1) generation of a single, consistent form of (P-P)Pd(0) complex for the ligands $Z(2,2), Z(2,3)$, MeOBiphep, $E(2,3)$, and $Z(3,3)$, which react directly with bromobenzene without prior dimerization of disproportionation and (2) generation of a distinct form of $(\mathrm{P}-\mathrm{P}) \mathrm{Pd}(0)$ complex for the ligand $Z(3,3)$ which similarly reacts directly with bromobenzene without prior dimerization of disproportionation.

\section{DFT calculations}

All calculations were performed with Gaussian 09.E; ${ }^{\text {s9 }}$ geometries were optimized at the B3LYP/6-31G(d) (LANL2DZ for Pd) level of DFT with THF-parameterized CPCM using the Berny algorithm. Ligand conformers were created as previously described. ${ }^{\mathrm{S} 10}$ Single-point energy was calculated at the M06-2L/6-311+G(d) (LANL2TZ for Pd) level in CPCM. We calculated the relationship between the tensile or compressive force acting between the $\mathrm{C}$ atoms of the $\mathrm{Me}$ groups of MeOBiphepPd(0) and of MeOBiphepPd(0)Cl- complexes and their geometrical parameters (Table S3) by relaxed potential-energy scans of the MeC...CMe distance. The restoring force of the constrained distance in such rPES is available directly from the Gaussian output of the converged geometry. We interpolated the resulting force vs. biphenO...Obiphen distance to $10 \mathrm{pN}$ increments and assumed that the same $\mathrm{O} \ldots \mathrm{O}$ distance in the externally constrained complex and its equivalent analog bearing the stiff-stilbene ligand means that stiff 
stilbene imposes the same force of the $\mathrm{Pd}($ biphen) core as external forces needed to constrain the $\mathrm{O} \ldots \mathrm{O}$ to the same value in stiff-stilbene free complex. (Scheme $\mathrm{S} 4) .{ }^{\mathrm{S} 10}$ We used this relationship as a calibration curve (Figure S11) to estimate the restoring force of the $\mathrm{O} \cdots \mathrm{O}$ coordinate in each stiff-stilbene containing (P-P)Pd(0) and (P-P)Pd(0)Cl ${ }^{-}$complex (Table S4). For the same ligand conformer the difference of the forces in the 2-coordinate and 3-coordinate complex was $<20 \mathrm{pN}$.
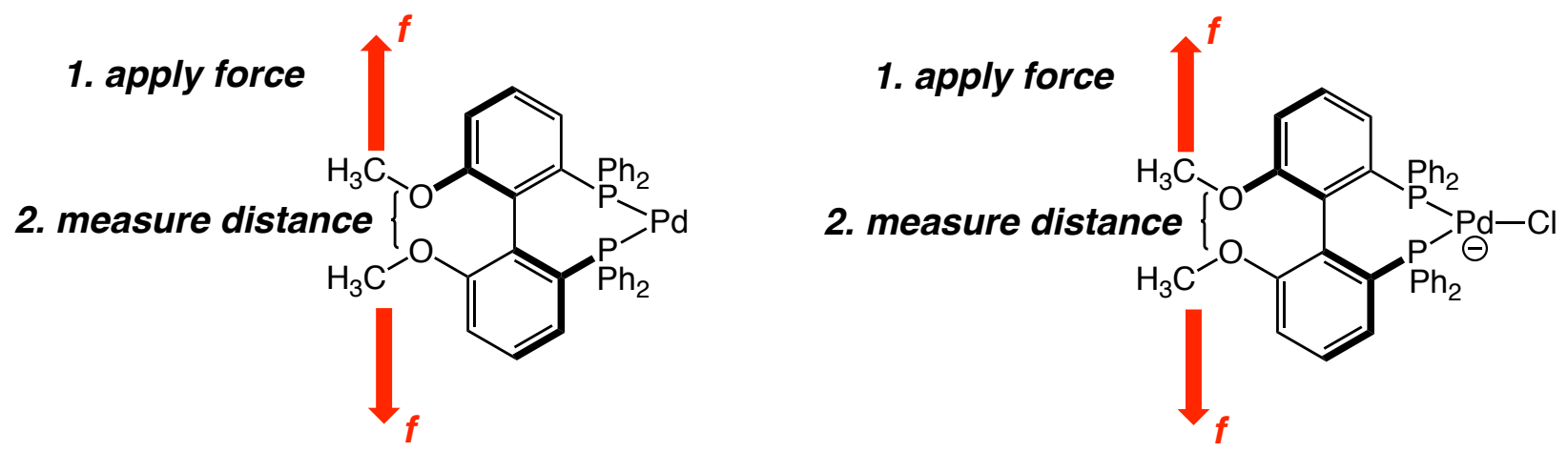

Scheme S4. Representation of the computational approach to correlate force applied to the carbon atoms of the $\mathrm{Me}$ groups and the $\mathrm{O} \cdots \mathrm{O}$ separation for (MeOBiphep) $\operatorname{Pd}(0)$ and (MeOBiphep) $\mathrm{Pd}(0) \mathrm{Cl}^{-}$complexes.

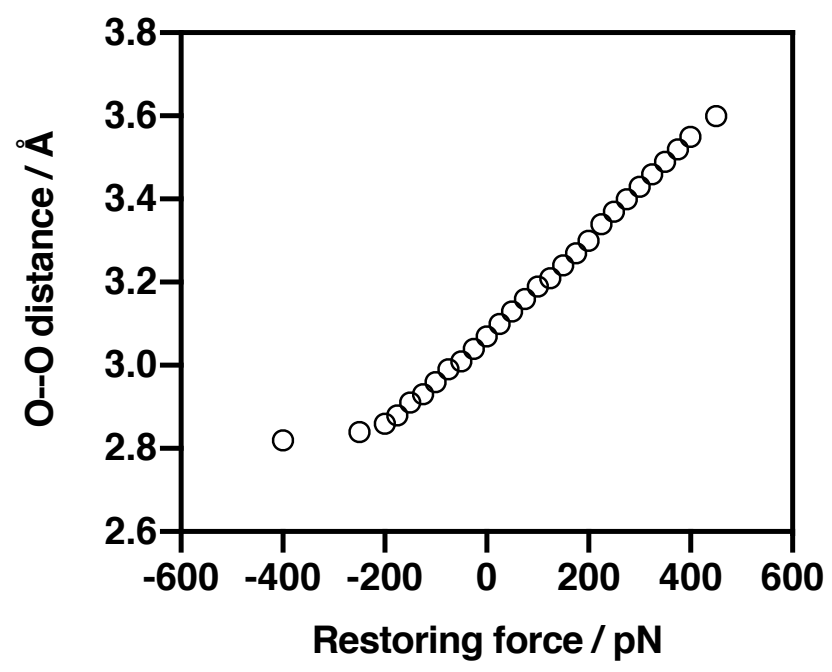

Figure S10. The calculated correlation between the applied force (between the $\mathrm{C}$ atoms of the Me groups) and the $\mathrm{O}$... O separation for the (P-P)Pd(0)Cl- complexes. 
Table S3. Converged $\mathrm{O} \cdots \mathrm{O}$ distances in two-coordinate (P-P)Pd and three-coordinate [(P$\mathrm{P}) \mathrm{Pd}] \mathrm{Cl}^{-}$palladium complexes of the ligands used to estimate their restoring forces.

\begin{tabular}{|c|c|c|c|}
\hline Ligand & relative energy & $\mathrm{O} \ldots \mathrm{O}[(\mathrm{P}-\mathrm{P}) \mathrm{Pd}](\AA)$ & $\mathrm{O} \ldots \mathrm{O}[(\mathrm{P}-\mathrm{P}) \mathrm{Pd}] \mathrm{Cl}-(\AA)$ \\
\hline $\mathrm{E} 32$ & 0 & 3.473022 & 3.389052 \\
\hline $\mathrm{E} 33 \mathrm{c} 1$ & 0 & 3.474408 & 3.408191 \\
\hline E33c2 & 0.17 & 3.506996 & - \\
\hline Z22 & 0 & 3.062258 & 3.011415 \\
\hline Z32c1 & 0 & 3.147593 & 3.050772 \\
\hline Z32c2 & 0.05 & 3.148426 & - \\
\hline Z32c3 & 0.54 & 3.219885 & - \\
\hline
\end{tabular}

Table S4. Calculated correlation between the $\mathrm{O} \cdots \mathrm{O}$ distance in $(\mathrm{MeOBiphep}) \mathrm{Pd}(0)$ and (MeOBiphep) $\mathrm{Pd}(0) \mathrm{Cl}^{-}$complexes versus the force applied across their ${ }_{\mathrm{Me}} \mathrm{C}$ atoms. This data comprises calibration curves used to estimate the restoring force of the $\mathrm{O} \cdots \mathrm{O}$ distance in the corresponding complexes of the reported ligands.

\begin{tabular}{|l|l|l|}
\hline Force, $\mathrm{pN}$ & (MeOBiphep)Pd & (MeOBiphep)PdCl- \\
\hline-400 & 2.857 & 2.823 \\
\hline-250 & 2.854 & 2.839 \\
\hline-200 & 2.898 & 2.861 \\
\hline-175 & 2.925 & 2.879 \\
\hline-150 & 2.945 & 2.905 \\
\hline-125 & 2.974 & 2.930 \\
\hline-100 & 3.000 & 2.959 \\
\hline-75 & 3.030 & 2.987 \\
\hline-50 & 3.060 & 3.014 \\
\hline-25 & 3.089 & 3.041 \\
\hline 0 & 3.116 & 3.069 \\
\hline 25 & 3.144 & 3.098 \\
\hline 50 & 3.175 & 3.127 \\
\hline 75 & 3.206 & 3.157 \\
\hline 100 & 3.236 & 3.185 \\
\hline 125 & 3.265 & 3.214 \\
\hline 150 & 3.294 & 3.244 \\
\hline 175 & 3.324 & 3.274 \\
\hline 200 & 3.355 & 3.304 \\
\hline 225 & 3.386 & 3.335 \\
\hline 250 & 3.418 & 3.367 \\
\hline 275 & 3.450 & 3.399 \\
\hline & & \\
\hline
\end{tabular}




\begin{tabular}{|l|l|l|}
\hline 300 & 3.483 & 3.431 \\
\hline 325 & 3.515 & 3.460 \\
\hline 350 & 3.545 & 3.489 \\
\hline 375 & 3.574 & 3.518 \\
\hline 400 & 3.604 & 3.546 \\
\hline
\end{tabular}

Table S5. Calculated electronic energies for (P-P)Pd(0) and (P-P)PdCl- complexes and estimates of $\Delta E / \Delta G$ for chloride dissociation from $(\mathrm{P}-\mathrm{P}) \mathrm{PdCl}^{-}$.

\begin{tabular}{|c|c|c|c|c|}
\hline $\mathrm{P}-\mathrm{P}$ & $(\mathrm{P}-\mathrm{P}) \mathrm{Pd}(0)$ & $(\mathrm{P}-\mathrm{P}) \mathrm{Pd}(0) \mathrm{Cl}^{-}$ & $\Delta \mathrm{E}(\mathrm{kcal} / \mathrm{mol})$ & $\Delta \mathrm{G}(\mathrm{kcal} / \mathrm{mol})$ \\
\hline $\mathrm{Z}(2,2)$ & -3349.7 & -3810.1 & -3.3 & -12.7 \\
\hline $\mathrm{Z}(2,3)$ & -3389.0 & -3849.4 & -4.1 & -13.6 \\
\hline MeOBiphep & -2427.4 & -2887.8 & -3.1 & -12.6 \\
\hline$E(2,3)$ & -3389.0 & -3849.4 & -1.8 & -11.2 \\
\hline$E(3,3)$ & -3428.3 & -3888.7 & -0.9 & -10.3 \\
\hline
\end{tabular}

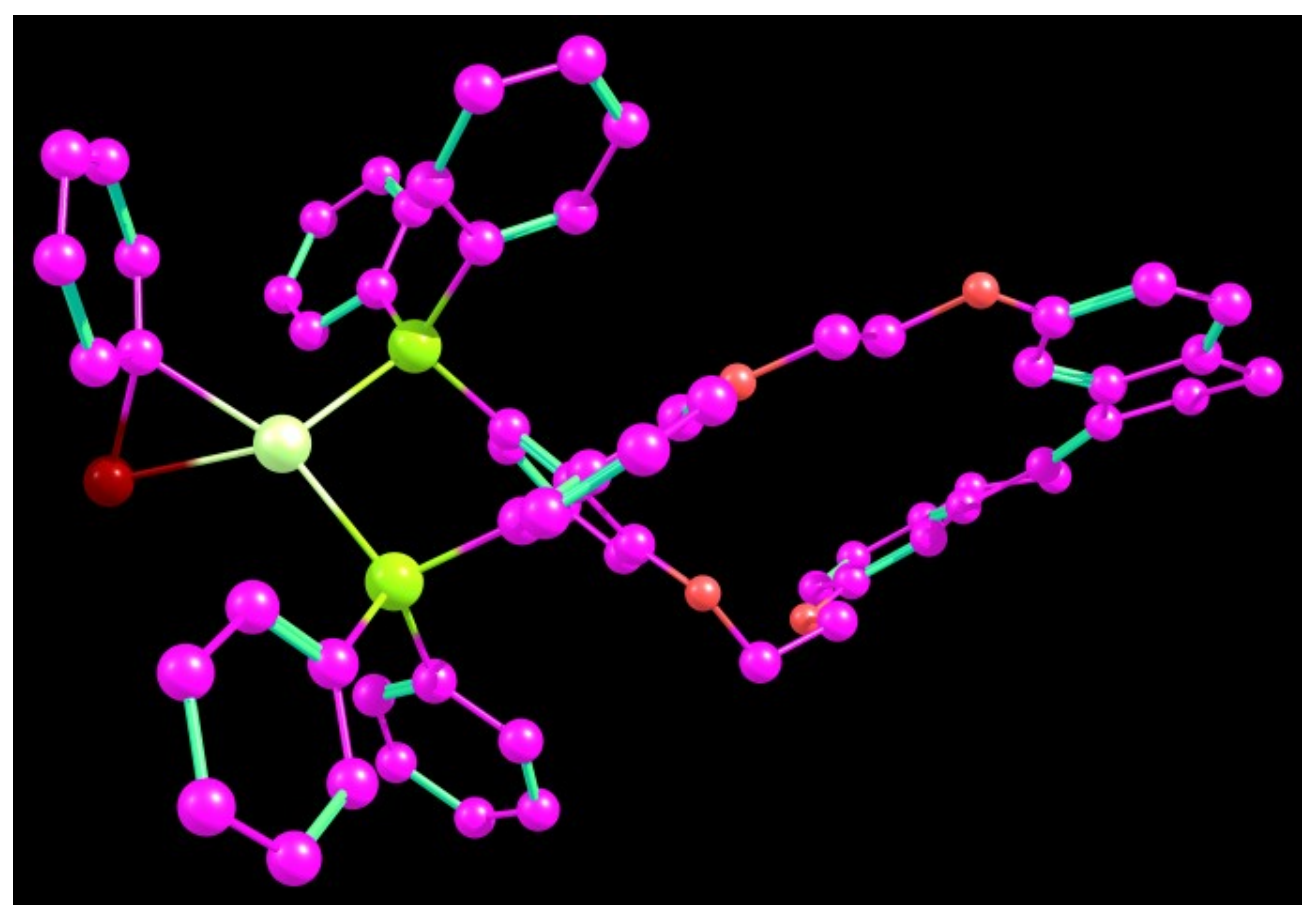

Figure S11. The optimized geometry of the transition state of oxidative addition of bromobenzene to $[Z(2,2)] \operatorname{Pd}(0)$. 
$\mathrm{J}(\mathrm{PP})$ and P-Pd-P angles of (dppe)Pd and (dppp)Pd complexes.

Table S6. ${ }^{2} J_{\mathrm{PP}}$ and $\mathrm{P}-\mathrm{Pd}-\mathrm{P}$ angles for structurally characterized $(\mathrm{P}-\mathrm{P}) \mathrm{Pd}(\mathrm{Ph}) \mathrm{X}$ complexes.

\begin{tabular}{|l|l|l|}
\hline Complexes & ${ }^{2} J_{\mathrm{PP}}(\mathrm{Hz})$ & $\mathrm{P}-\mathrm{Pd}-\mathrm{P}\left(^{\circ}\right)^{\mathrm{S} 13}$ \\
\hline$(\mathrm{dppe}) \mathrm{Pd}(\mathrm{Ph}) I^{\mathrm{S} 11}$ & 27.1 & 85.03 \\
\hline$(\mathrm{dppp}) \mathrm{Pd}(\mathrm{Ph}) \mathrm{Br}^{\mathrm{S} 12}$ & 54.0 & 91.08 \\
\hline
\end{tabular}

\section{Crystal structures and data}

Table s7. Crystal data and structure refinement for $[\mathrm{Z}(3,3)] \mathrm{PdCl}_{2} \bullet \mathrm{Et}_{2} \mathrm{O} / \mathrm{C}_{6} \mathrm{H}_{5} \mathrm{Cl}$ and (MeOBiphep)PdCl${ }_{2} \bullet \mathrm{CH}_{3} \mathrm{CN}$.

\begin{tabular}{|c|c|c|}
\hline & $\mathrm{Z}(3,3) \mathrm{PdCl}_{2}$ & (MeOBiphep) $\mathrm{PdCl}_{2}$ \\
\hline CCDC & 1995519 & 1995520 \\
\hline Empirical formula & $\mathrm{C}_{69} \mathrm{H}_{68} \mathrm{Cl}_{2.50} \mathrm{O}_{5.50} \mathrm{P}_{2} \mathrm{Pd}$ & $\mathrm{C}_{40} \mathrm{H}_{35} \mathrm{Cl}_{2} \mathrm{NO}_{2} \mathrm{P}_{2} \mathrm{Pd}$ \\
\hline Formula weight & $1242.19 \mathrm{~g} / \mathrm{mol}$ & $800.93 \mathrm{~g} / \mathrm{mol}$ \\
\hline Temperature & $100(2) \mathrm{K}$ & $100(2) \mathrm{K}$ \\
\hline Wavelength & $1.54178 \AA$ & $0.71073 \AA$ \\
\hline Crystal system & triclinic & monoclinic \\
\hline Space group & $P-1$ & P 1211 \\
\hline Unit cell dimensions & $\begin{array}{l}a=14.1420(5) \AA \\
b=14.6244(5) \AA \\
c=15.2201(5) \AA \\
\alpha=100.0700(10)^{\circ} \\
\beta=104.0960(10)^{\circ} \\
\gamma=90.5090(10)^{\circ}\end{array}$ & $\begin{array}{l}a=11.8149(7) \AA \\
b=12.5111(8) \AA \\
c=12.1216(8) \AA \\
\alpha=90^{\circ} \\
\beta=102.2010(15)^{\circ} \\
\gamma=90^{\circ}\end{array}$ \\
\hline Volume & $3001.61(18) \AA^{3}$ & $1751.31(19) \AA^{3}$ \\
\hline $\bar{Z}$ & 2 & 2 \\
\hline Density (calculated) & $1.374 \mathrm{~g} / \mathrm{cm}^{3}$ & $1.519 \mathrm{~g} / \mathrm{cm}^{3}$ \\
\hline Absorption coefficient & $4.430 \mathrm{~mm}^{-1}$ & $0.811 \mathrm{~mm}^{-1}$ \\
\hline $\mathrm{F}(000)$ & 1289 & 816 \\
\hline Crystal size & $0.034 \times 0.227 \times 0.373 \mathrm{~mm}$ & $0.081 \times 0.148 \times 0.209 \mathrm{~mm}$ \\
\hline Reflections collected & 106238 & 43727 \\
\hline
\end{tabular}




\begin{tabular}{|l|l|l|}
\hline Independent reflections & $12938[\mathrm{R}(\mathrm{int})=0.0410]$ & $14729[\mathrm{R}(\mathrm{int})=0.0498]$ \\
\hline $\begin{array}{l}\text { Data/ restraints/ } \\
\text { parameters }\end{array}$ & $12938 / 1187 / 1107$ & $14729 / 1 / 436$ \\
\hline Goodness-of-fit on $\mathrm{F}^{2}$ & 1.042 & 1.001 \\
\hline $\begin{array}{l}\text { Final } \mathrm{R} \text { indices } \\
{[\mathrm{l} \text { 2sigma(I)] }}\end{array}$ & $\mathrm{R} 1=0.0380, \mathrm{wR} 2=0.1003$ & $\mathrm{R} 1=0.0351, \mathrm{wR} 2=0.0612$ \\
\hline $\mathrm{R}$ indices (all data) & $\mathrm{R} 1=0.0398, \mathrm{wR} 2=0.1025$ & $\mathrm{R} 1=0.0460, \mathrm{wR} 2=0.0649$ \\
\hline
\end{tabular}

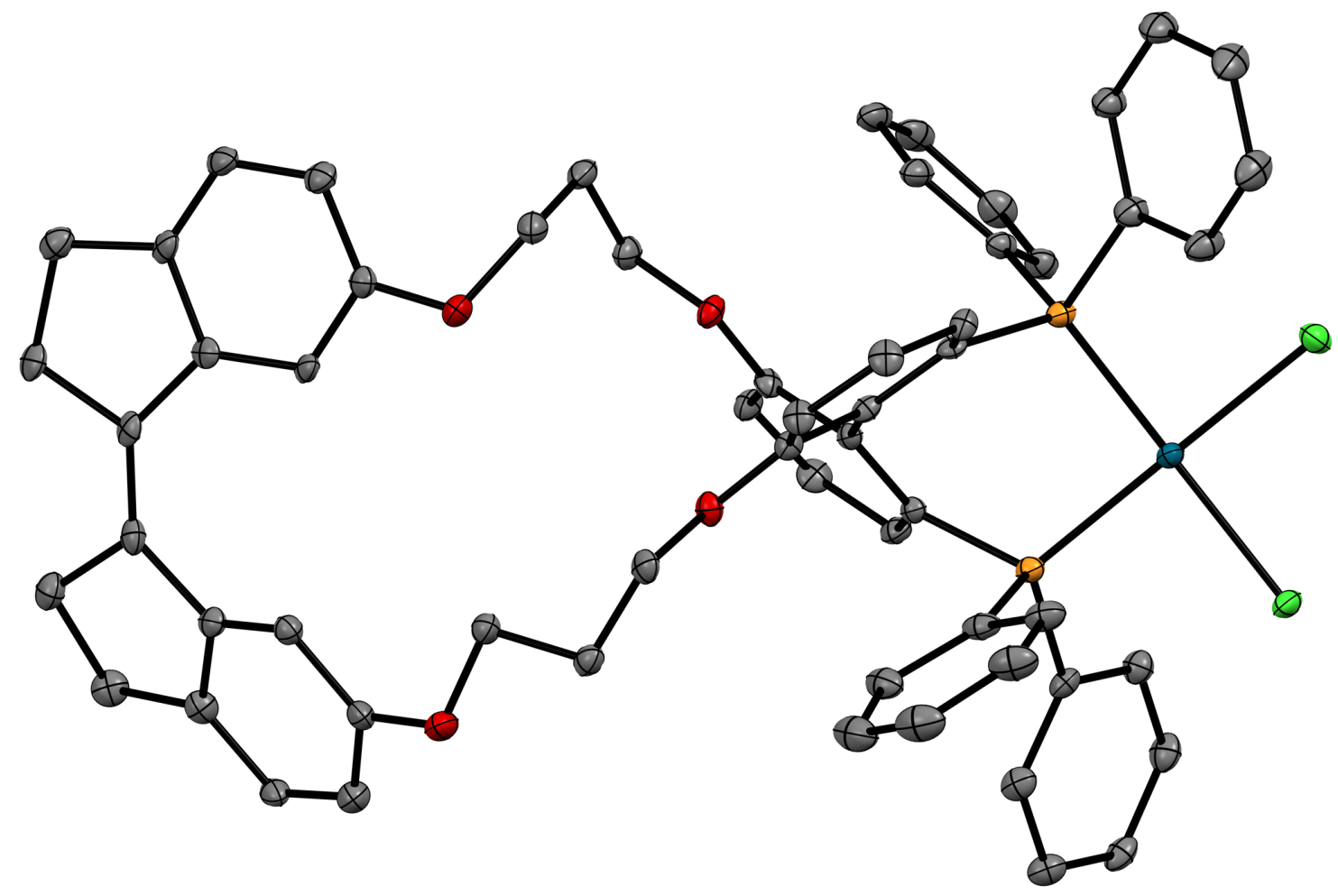

Figure S12. The ORTEP diagram of $[\mathrm{Z}(3,3)] \mathrm{PdCl}_{2} \bullet \mathrm{Et}_{2} \mathrm{O} / \mathrm{C}_{6} \mathrm{H}_{5} \mathrm{Cl}$. with ellipsoids shown at the $50 \%$ probability level and with counterion, solvent, and hydrogen atoms omitted for clarity. Selected bond distances $(\AA)$, bond angles (deg) and torsions (deg): Cl2-Pd = 2.3456(7), Cl1-Pd = 2.3550(6), Pd-P2 = 2.2559(7), Pd-P1 = 2.2623(6), Cl2-Pd-Cl1 = 91.54(2), P1-Pd-Cl2 = 90.80(2), $\mathrm{P} 1-\mathrm{Pd}-\mathrm{P} 2$ = 92.37(2), P2-Pd-Cl1 = 89.59(2), C7-C8-C2-C1 = 72(2). 


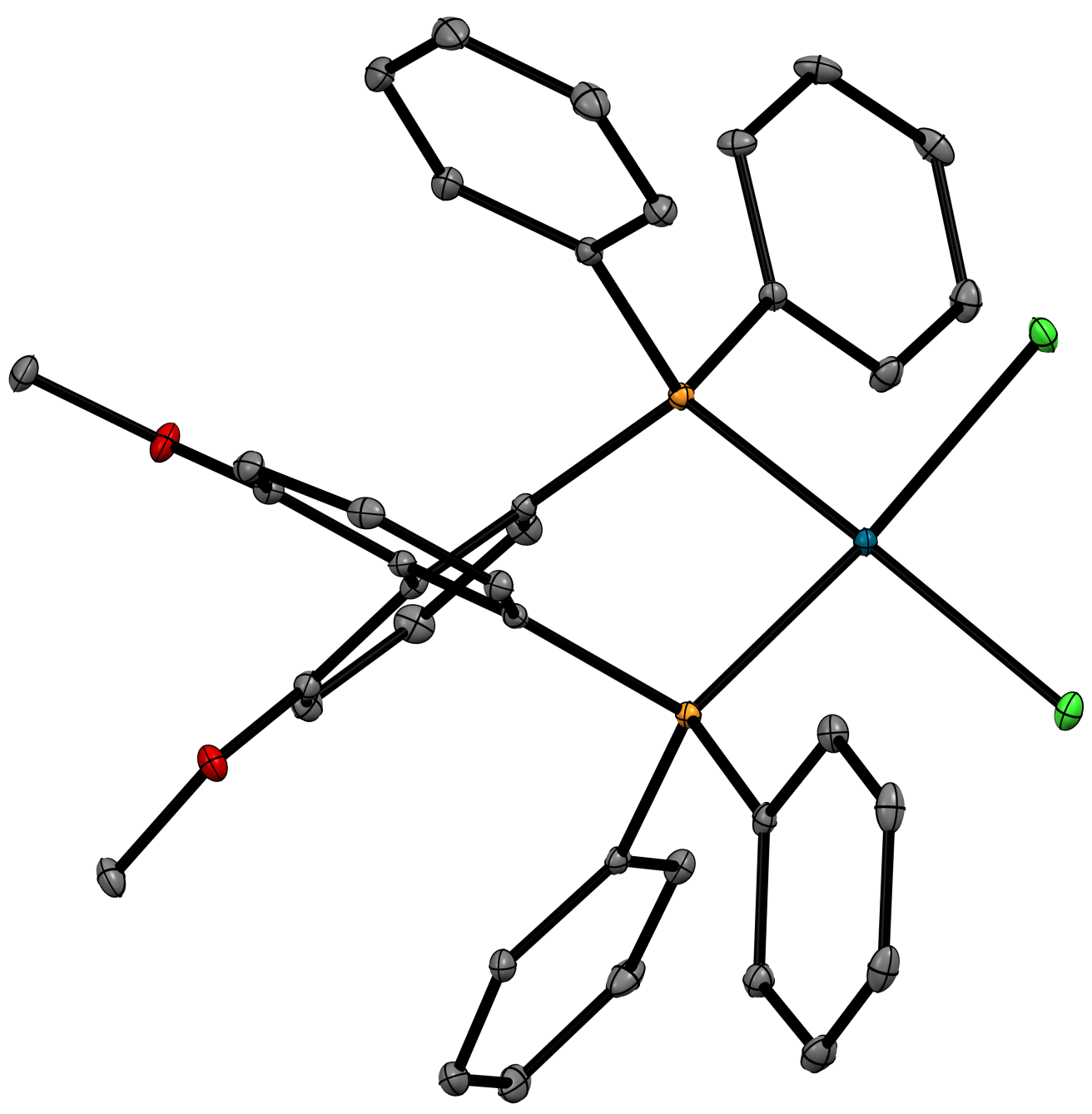

Figure S13. The ORTEP diagram of (MeOBiphep) $\mathrm{PdCl}_{2} \cdot \mathrm{CH}_{3} \mathrm{CN}$ with ellipsoids shown at the $50 \%$ probability level and with counterion, solvent, and hydrogen atoms omitted for clarity. Selected bond distances $(\AA)$, bond angles (deg) and torsions (deg): $\mathrm{Pd}-\mathrm{Cl} 2=2.3605(7), \mathrm{Pd}-\mathrm{Cl} 1$ = 2.3608(8), Pd-P1 = 2.2681(8), Pd-P2 = 2.2502(8), Cl1-Pd-Cl2 = 90.33(3), Cl1-Pd-P1 = 89.99(3), $\mathrm{P} 1-\mathrm{Pd}-\mathrm{P} 2$ = 92.13(3), P2-Pd-Cl2 = 90.63(3), C7-C8-C6-C1 = 68.8(4). 


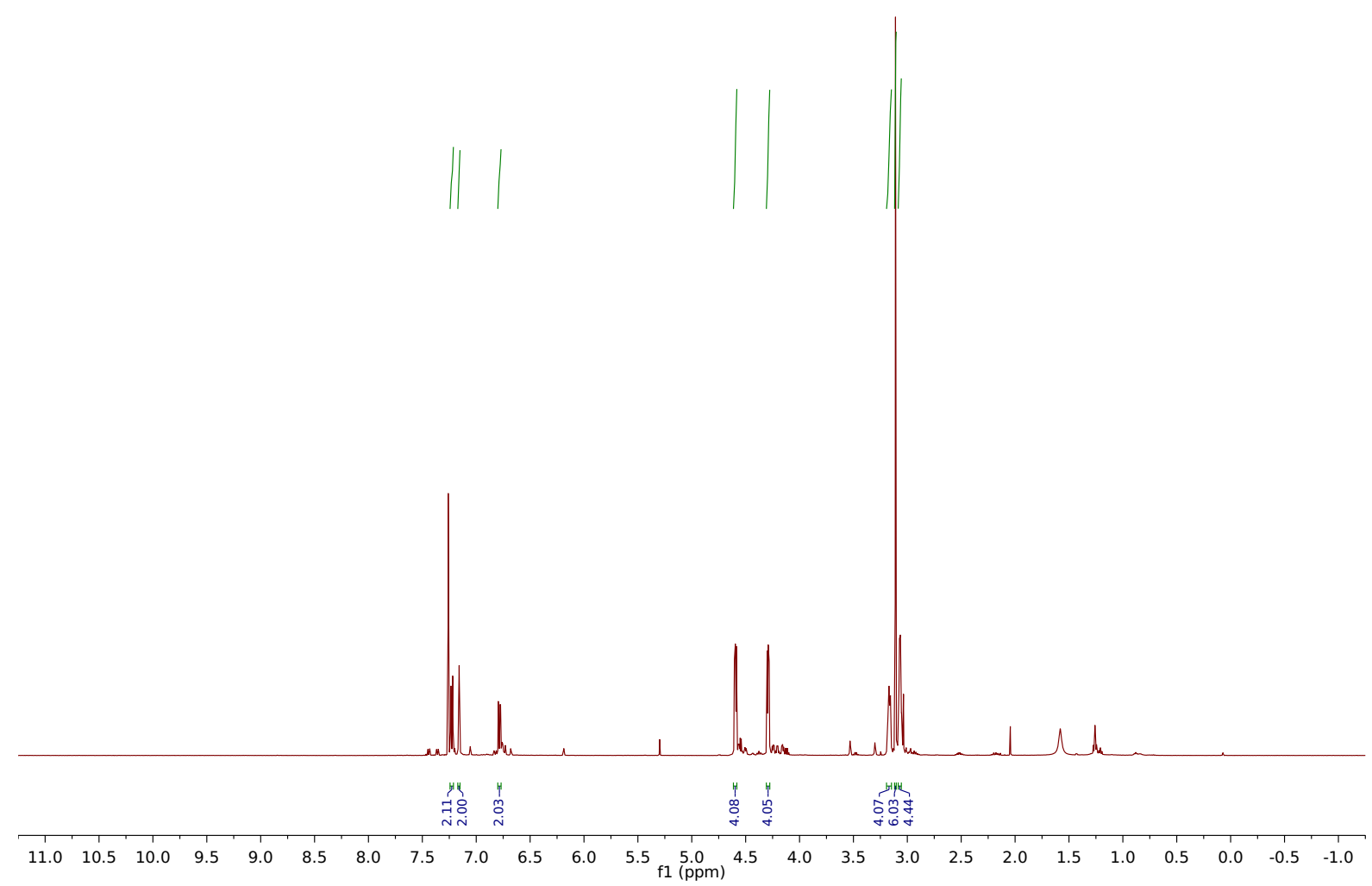

Figure S14. ${ }^{1} \mathrm{H}$ NMR spectrum of S3. 


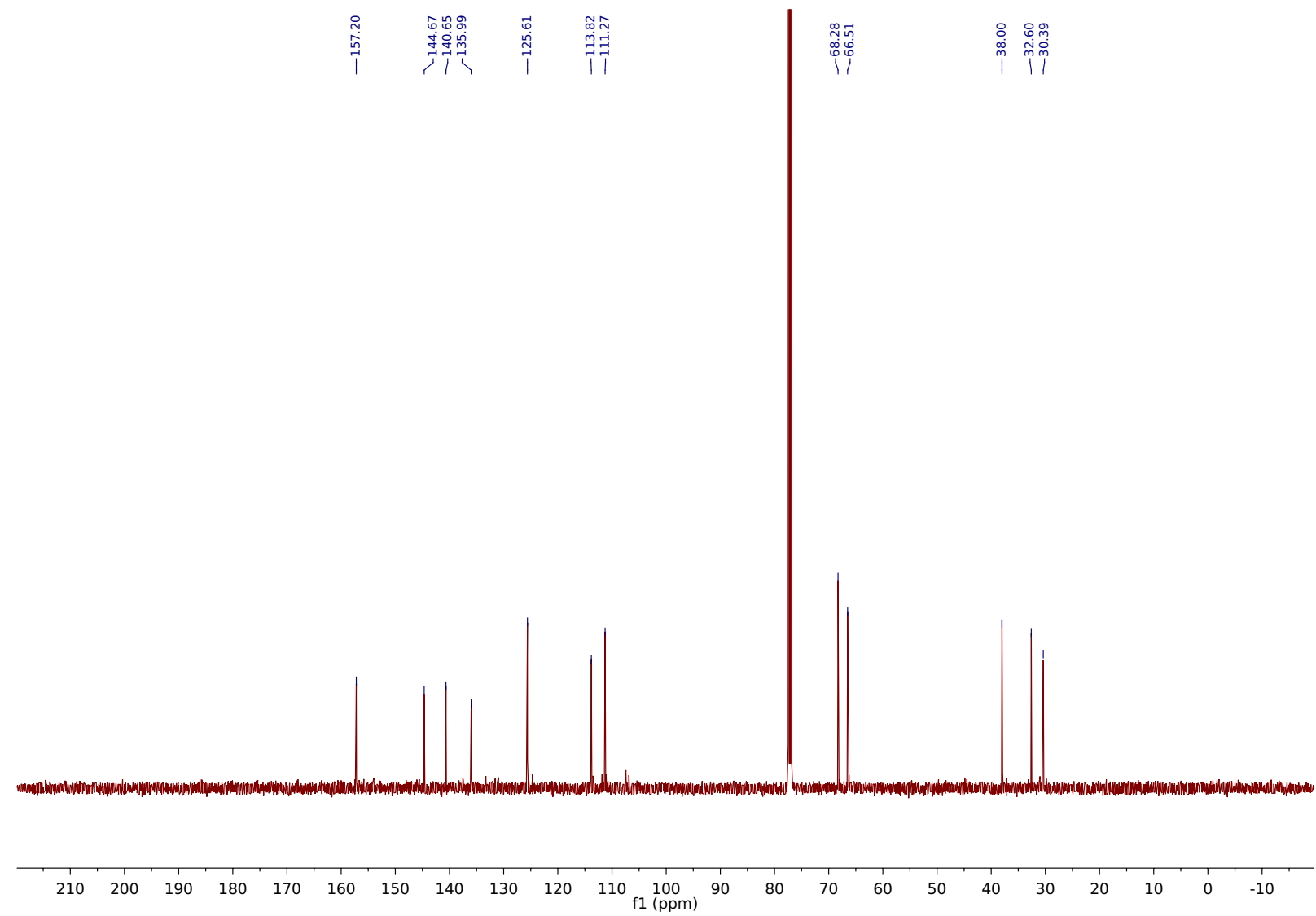

Figure S15. ${ }^{13} \mathrm{C}$ NMR spectrum of S3. 


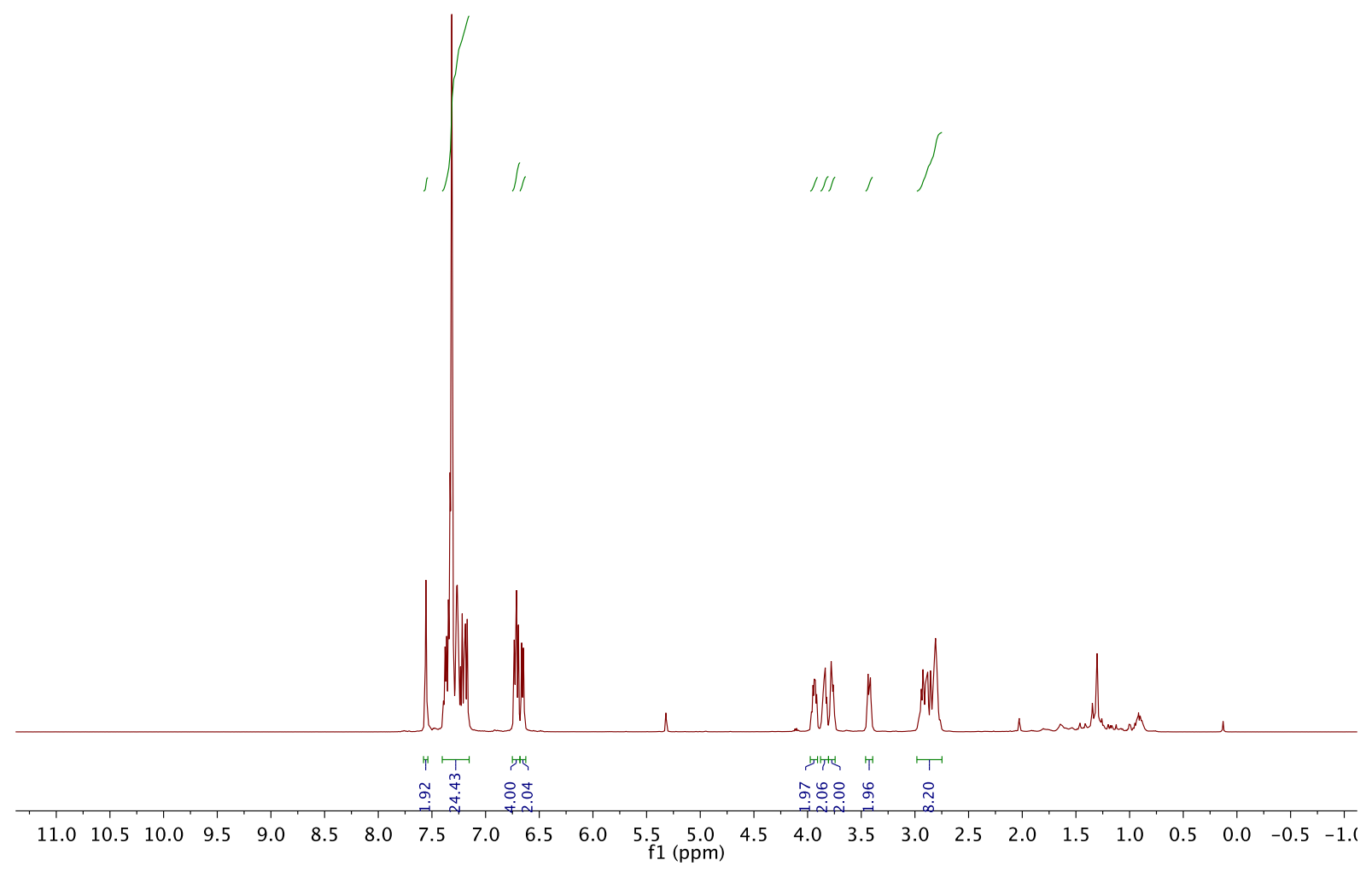

Figure S16. ${ }^{1} \mathrm{H}$ NMR spectrum of $Z \mathbf{Z}(2,2)$. 


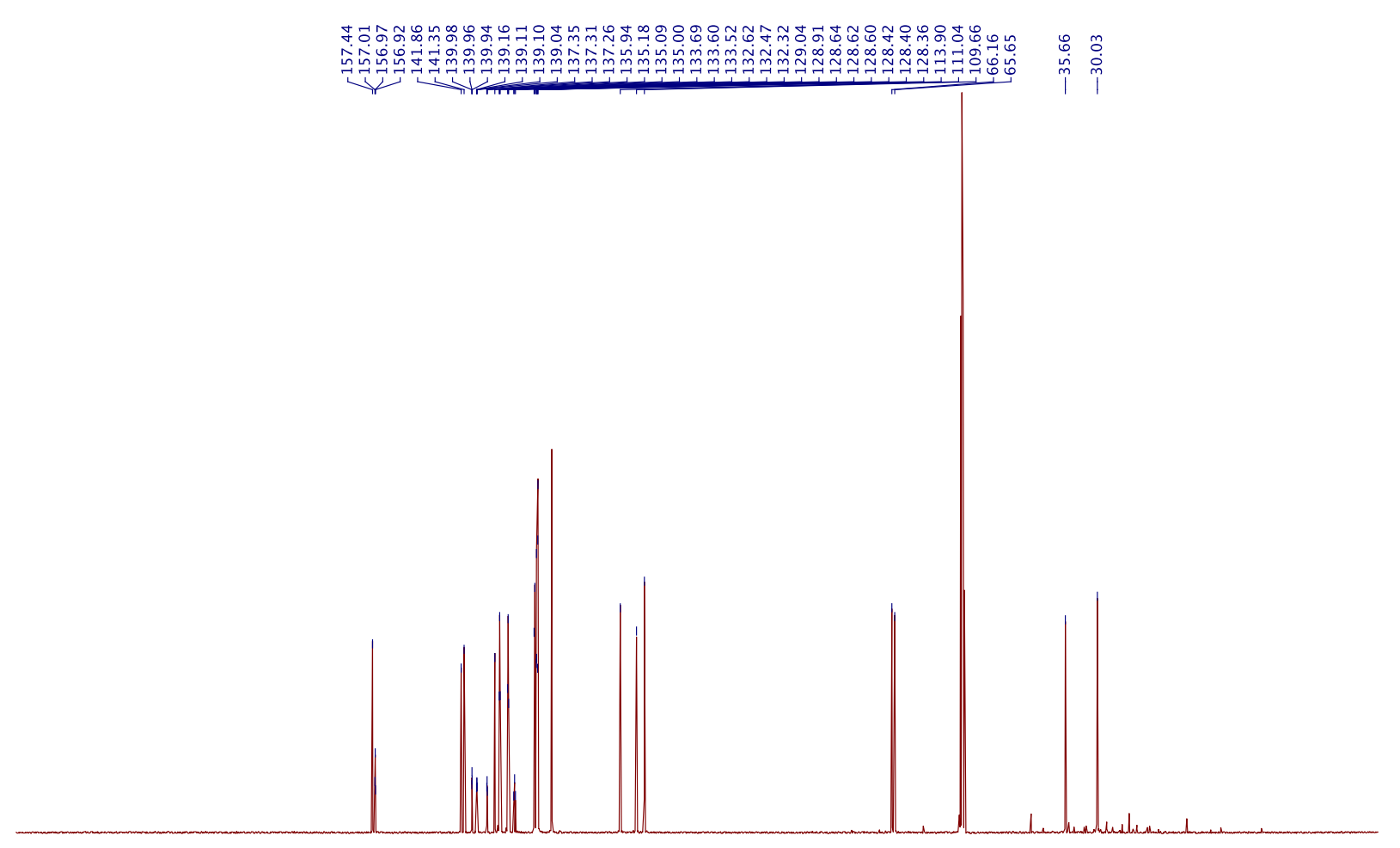

$\begin{array}{lllllllllllllllllllllllllllll}30 & 210 & 200 & 190 & 180 & 170 & 160 & 150 & 140 & 130 & 120 & 110 & 100 & 90 & 80 & 70 & 60 & 50 & 40 & 30 & 20 & 10 & 0 & -10\end{array}$

Figure S17. ${ }^{13} \mathrm{C}$ NMR spectrum of $Z(2,2)$. 


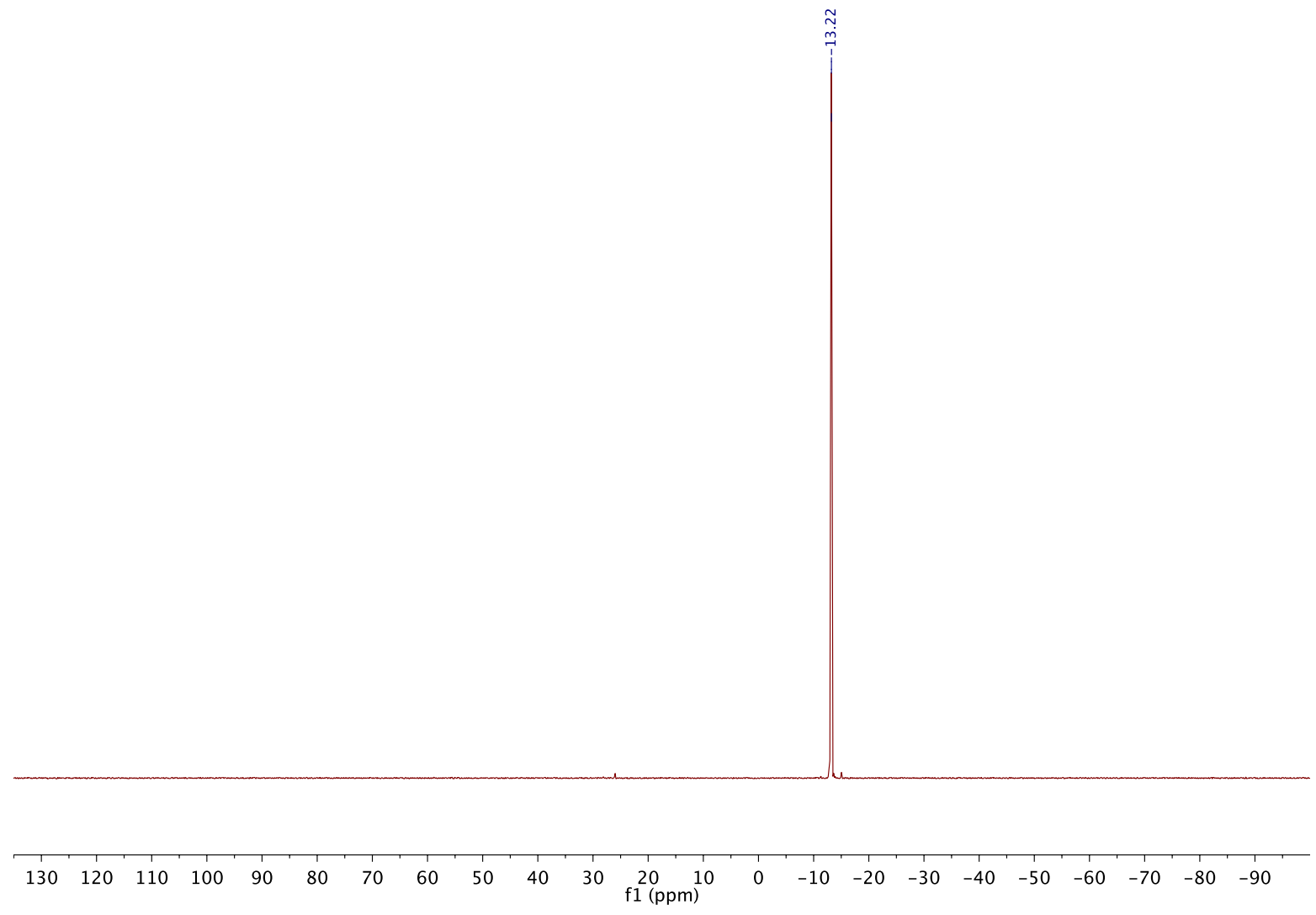

Figure S18. ${ }^{31} \mathrm{P}$ NMR spectrum of $\mathbf{Z}(2,2)$. 


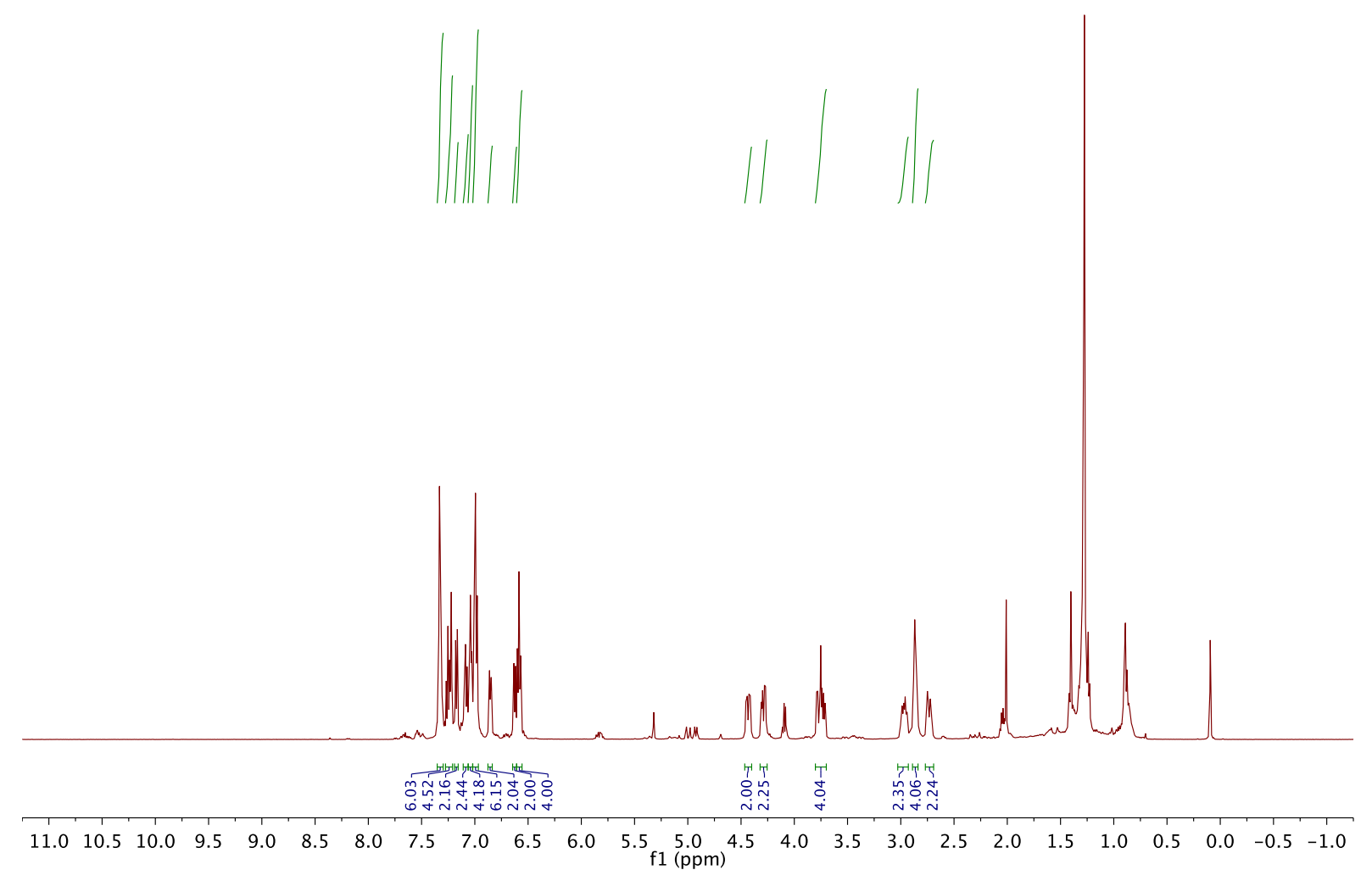

Figure S19. ${ }^{1} \mathrm{H}$ NMR spectrum of $E(2,2)$. 


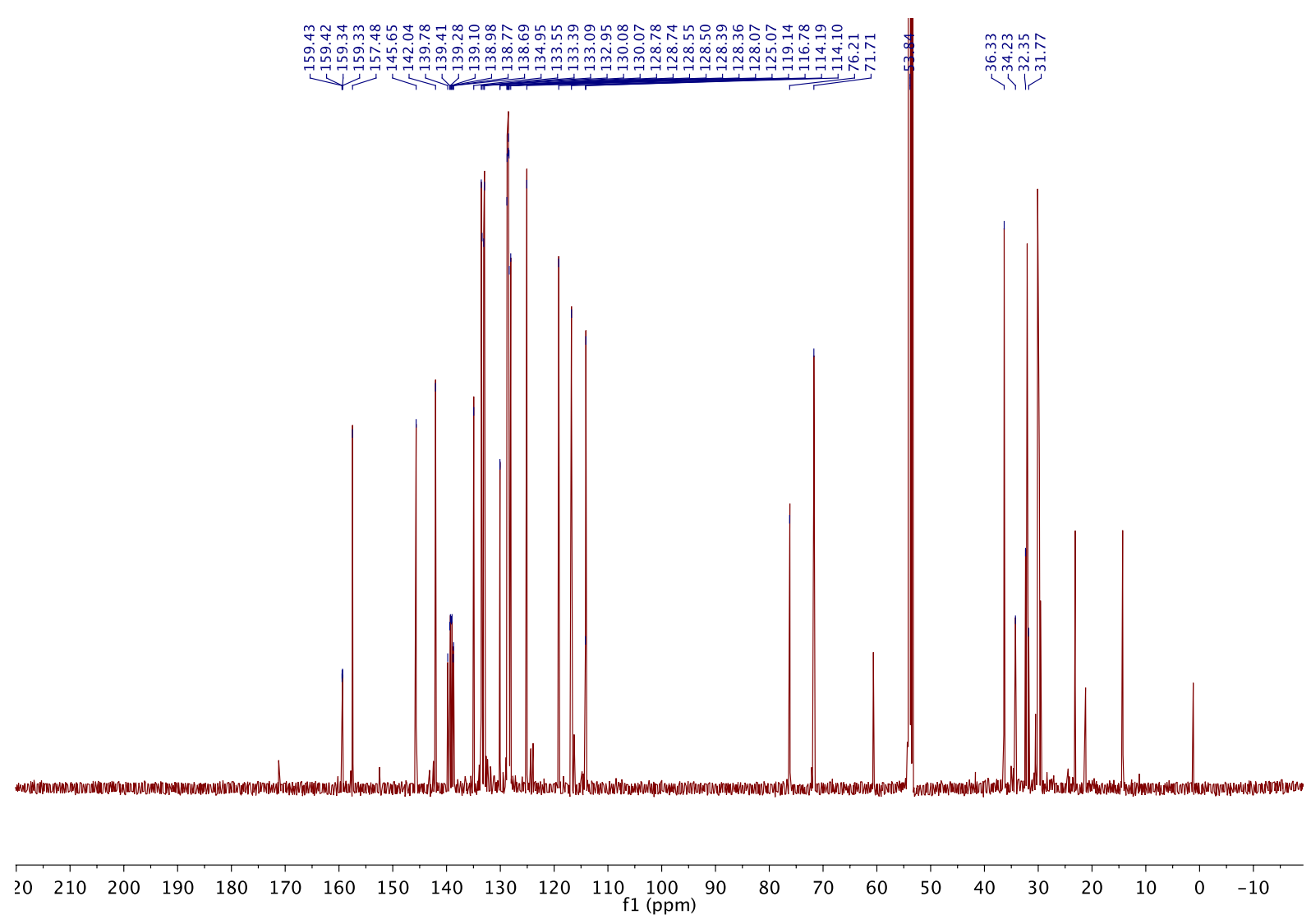

Figure S20. ${ }^{13} \mathrm{C}$ NMR spectrum of $E(2,2)$. 


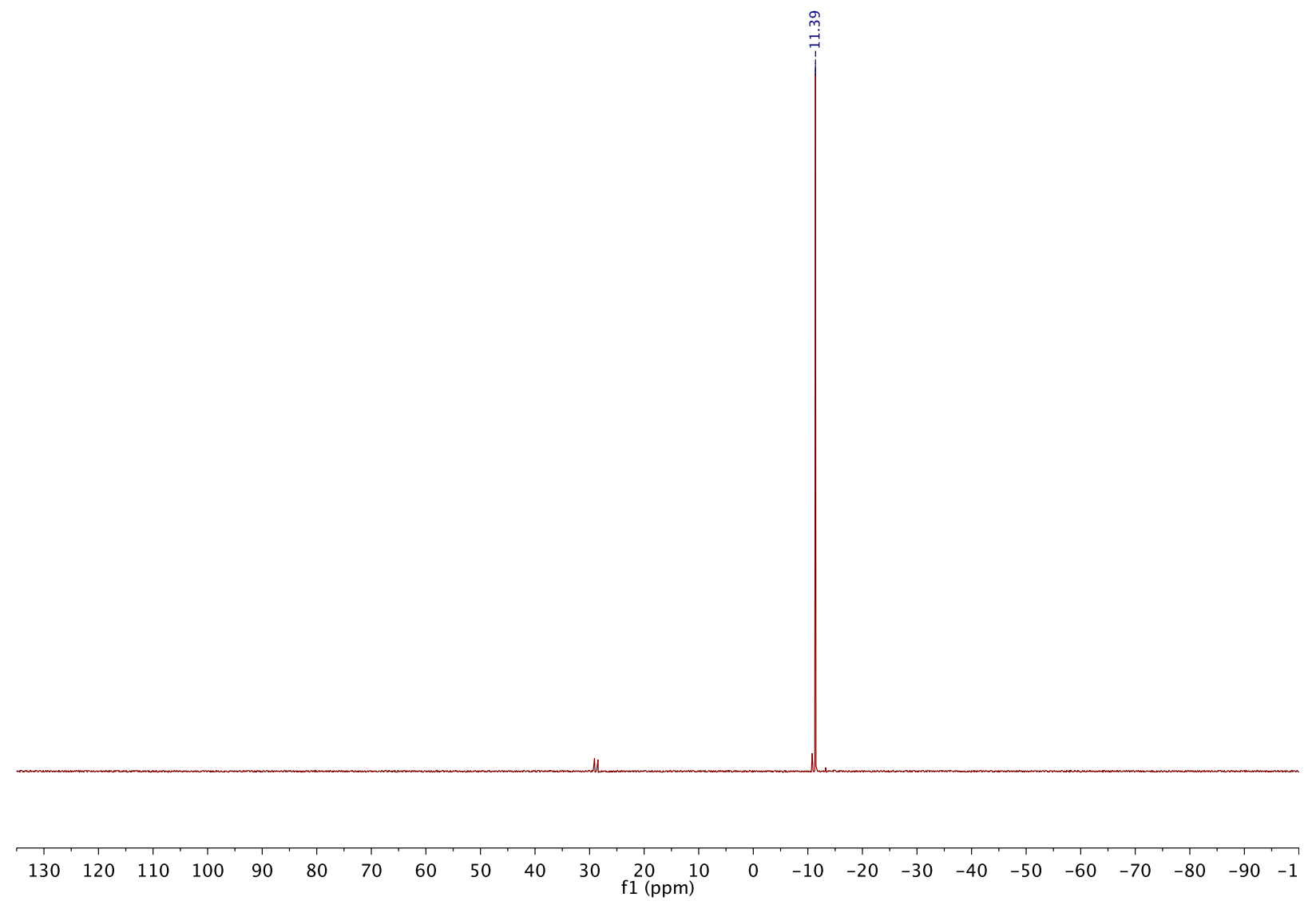

Figure S21. ${ }^{31} \mathrm{P}$ NMR spectrum of $E(2,2)$. 


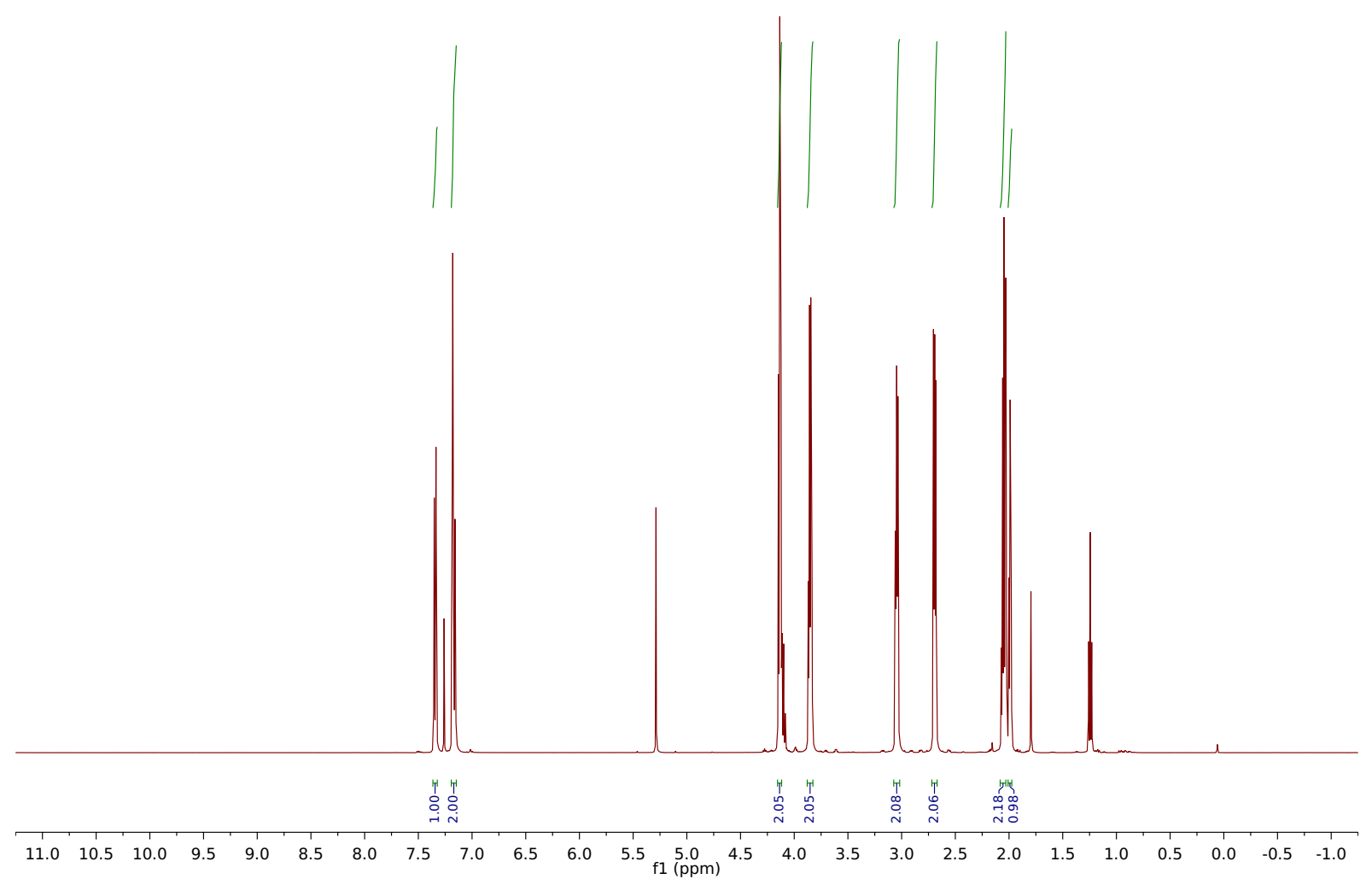

Figure S22. ${ }^{1} \mathrm{H}$ NMR spectrum of $\mathbf{S 4}$. 


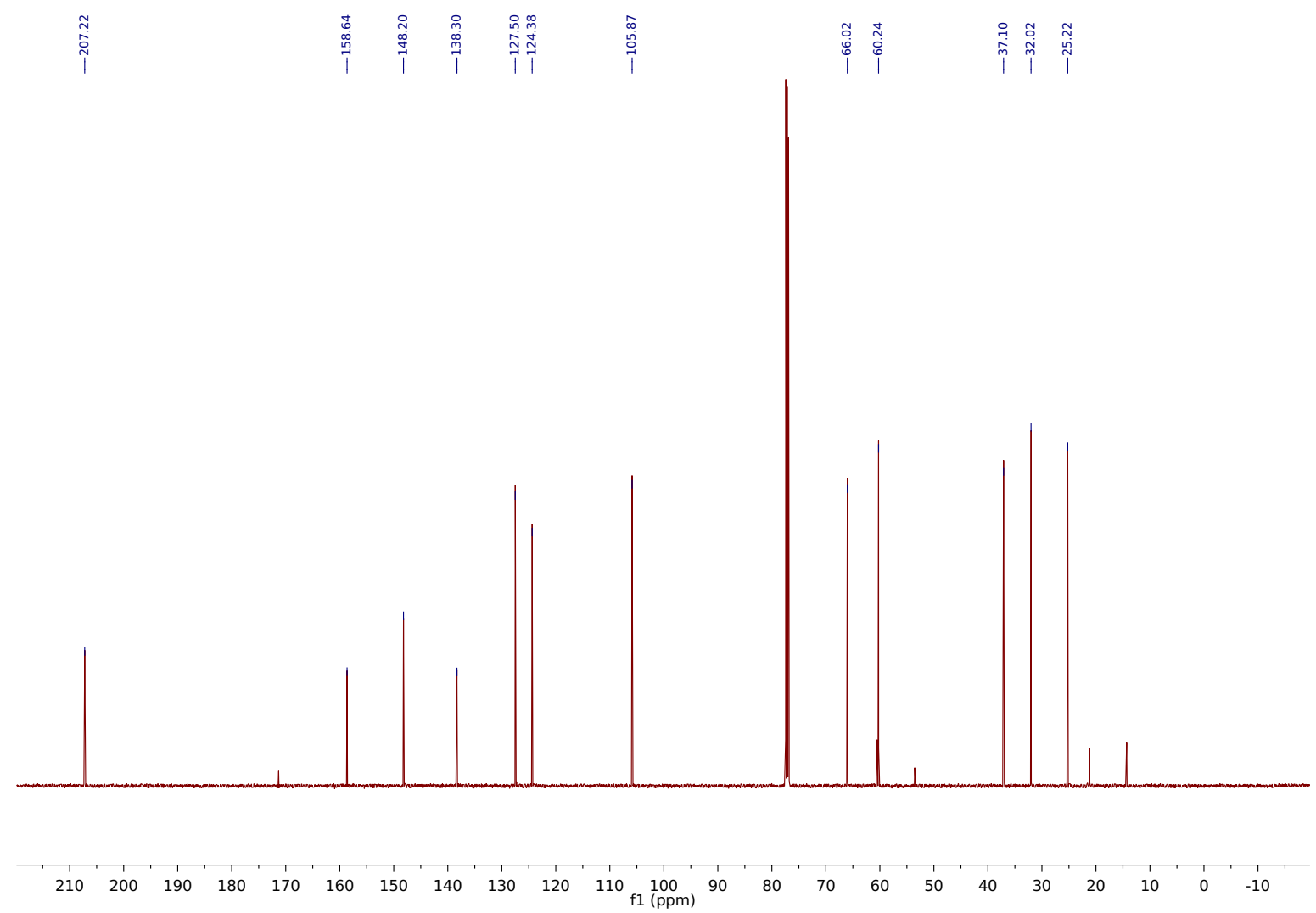

Figure S23. ${ }^{13} \mathrm{C}$ NMR spectrum of S4. 


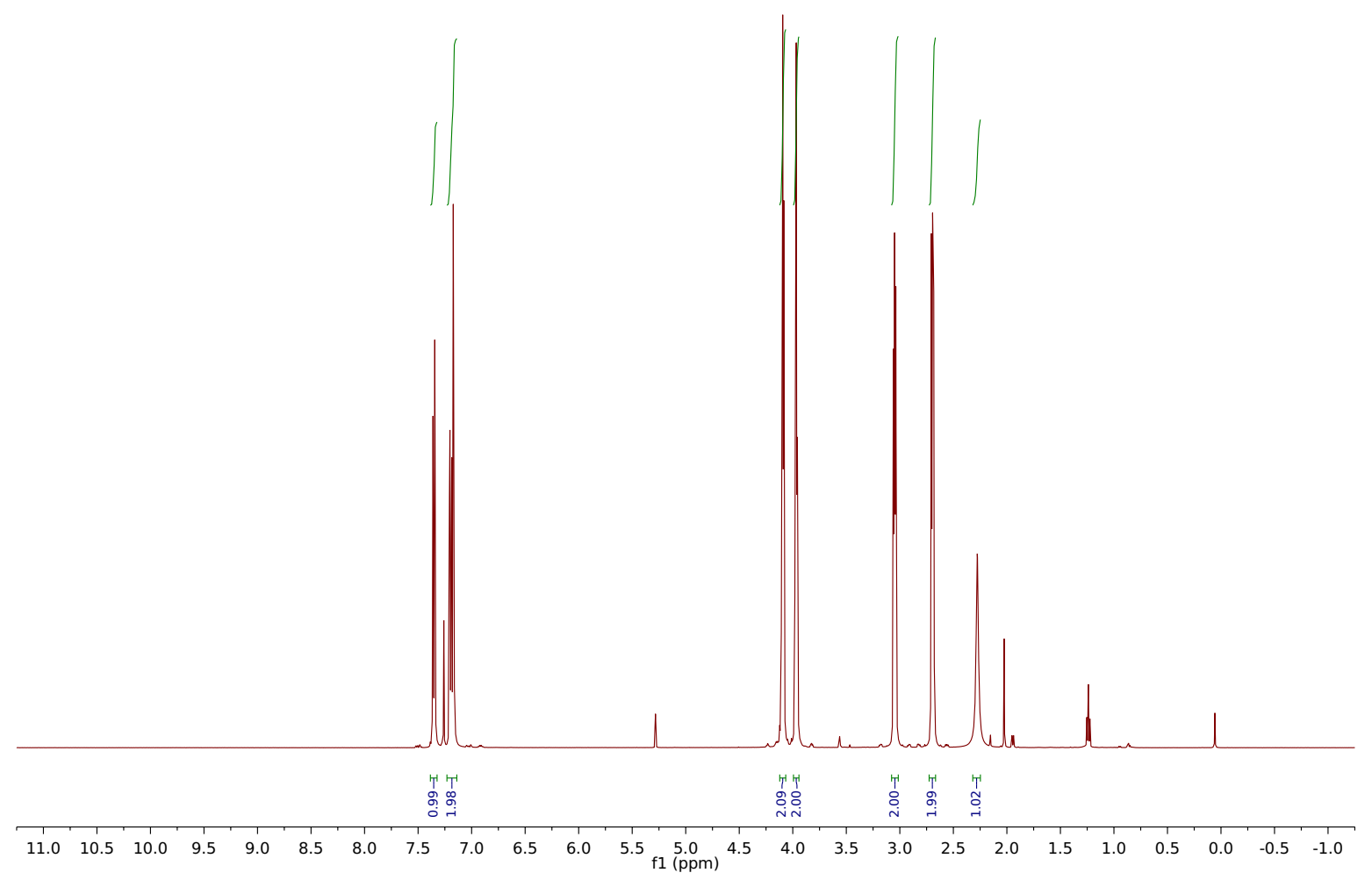

Figure S24. ${ }^{1} \mathrm{H}$ NMR spectrum of $\mathbf{S 5}$. 


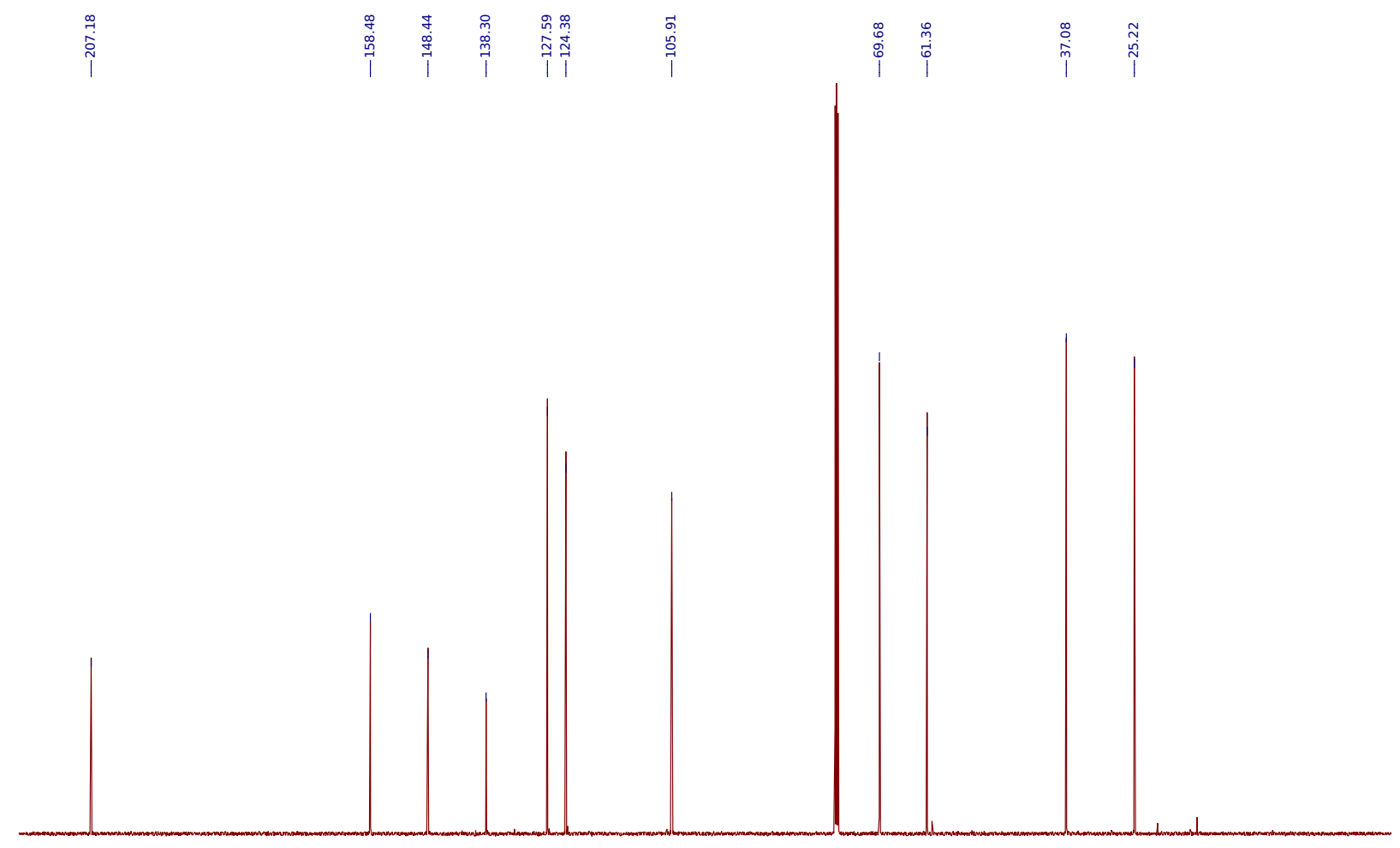

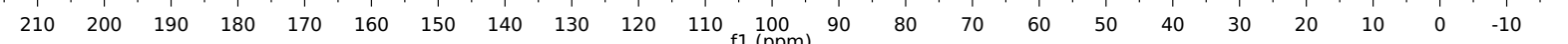

Figure S25. ${ }^{13} \mathrm{C}$ NMR spectrum of S5. 


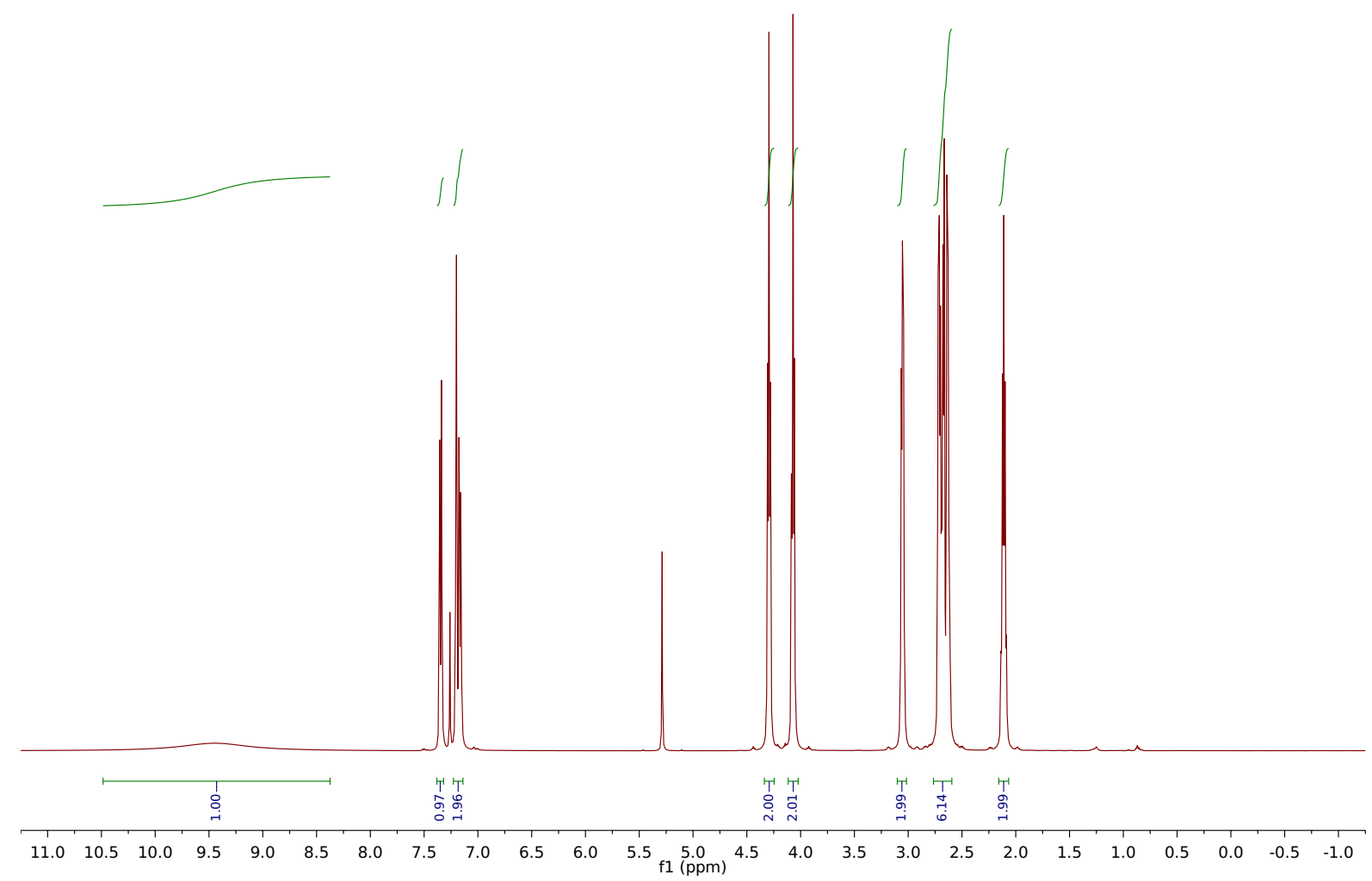

Figure S26. ${ }^{1} \mathrm{H}$ NMR spectrum of $\mathbf{S 6}$. 


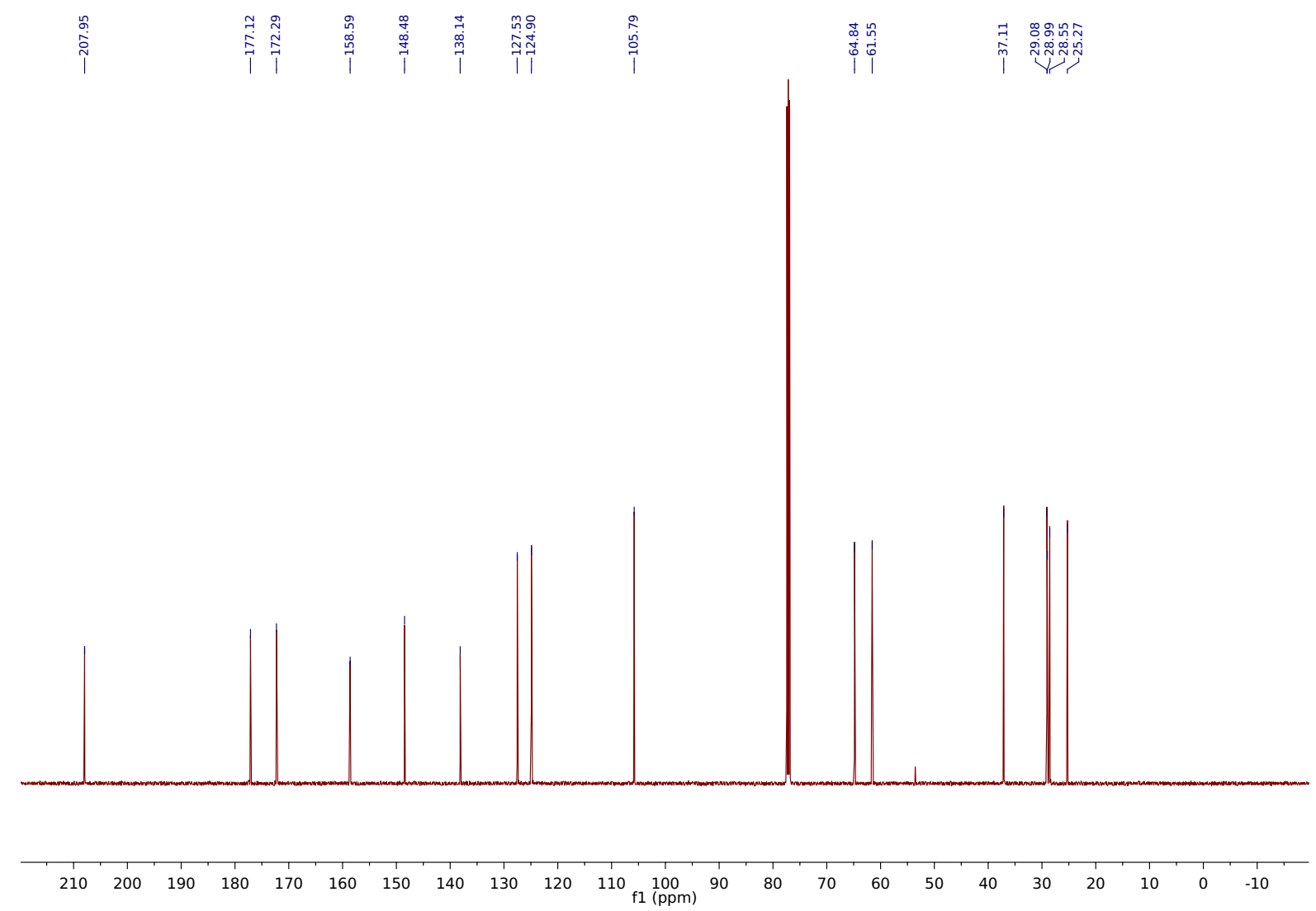

Figure S27. ${ }^{13} \mathrm{C}$ NMR spectrum of $\mathbf{S 6}$. 


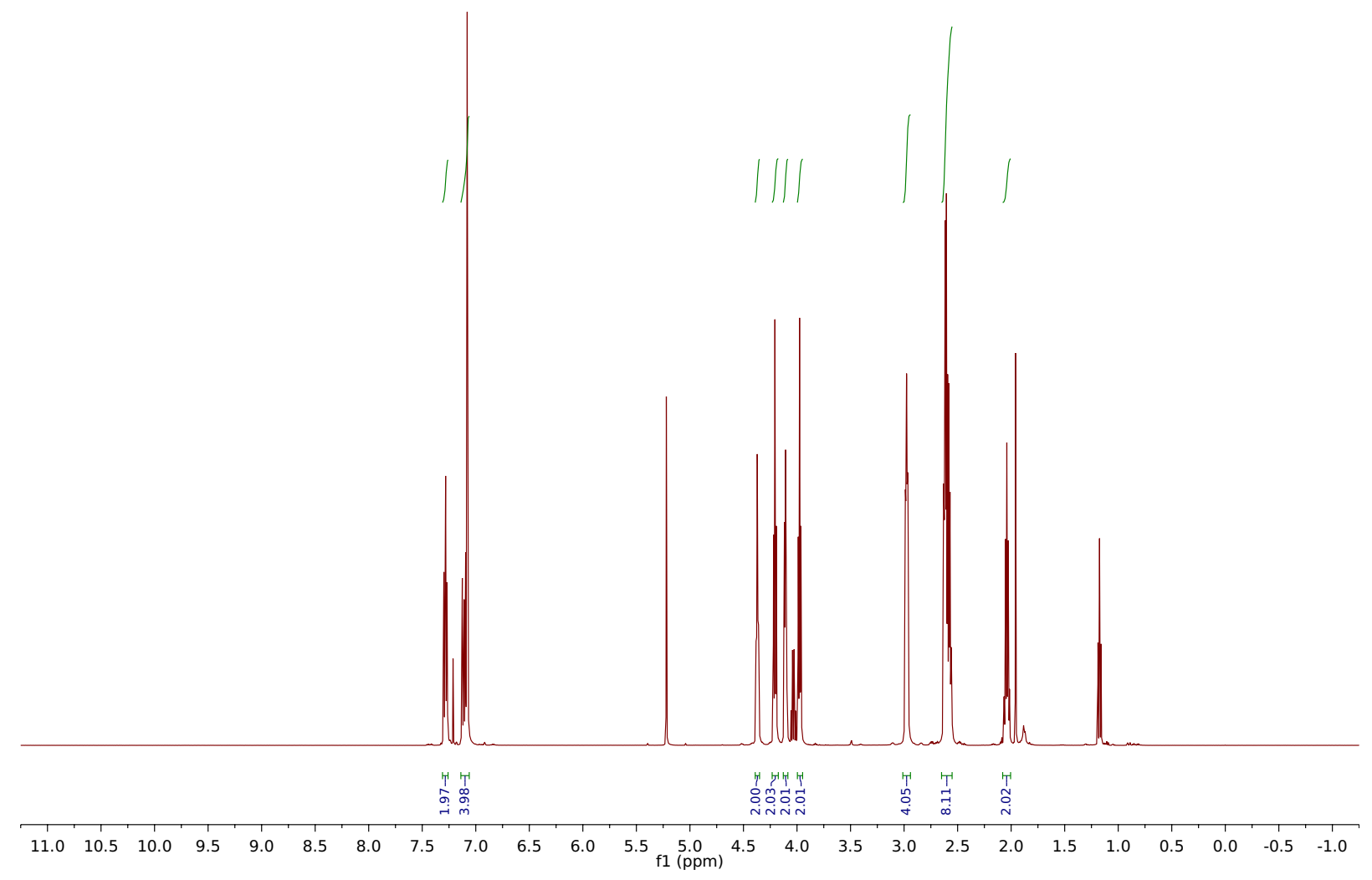

Figure S28. ${ }^{1} \mathrm{H}$ NMR spectrum of S7. 

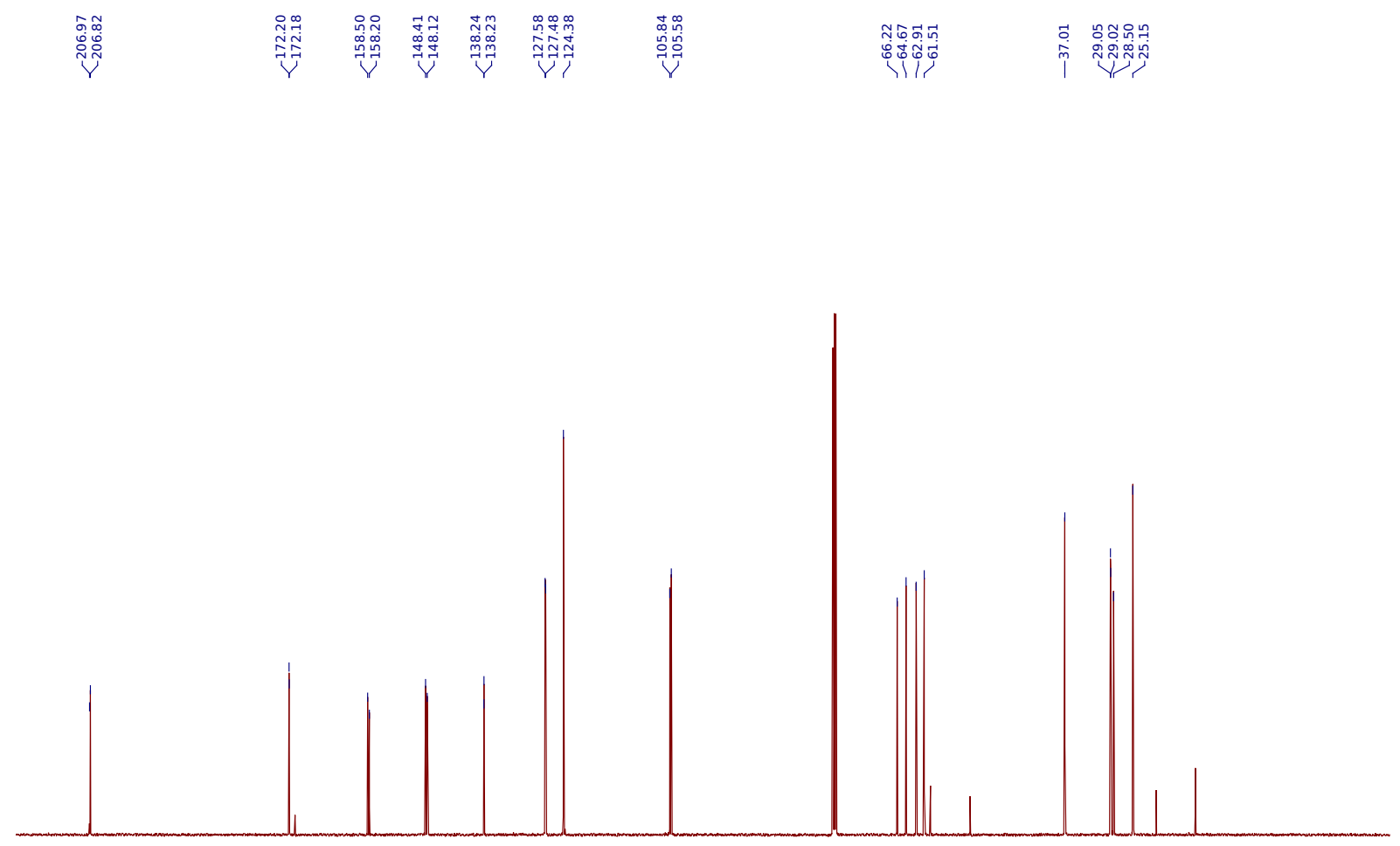

$\begin{array}{lllllllllllllllllllllll}210 & 200 & 190 & 180 & 170 & 160 & 150 & 140 & 130 & 120 & 110 & 100 & 100 & 80 & 70 & 60 & 50 & 40 & 30 & 20 & 10 & 0 & -10\end{array}$

Figure S29. ${ }^{13} \mathrm{C}$ NMR spectrum of $\mathbf{S 7}$. 


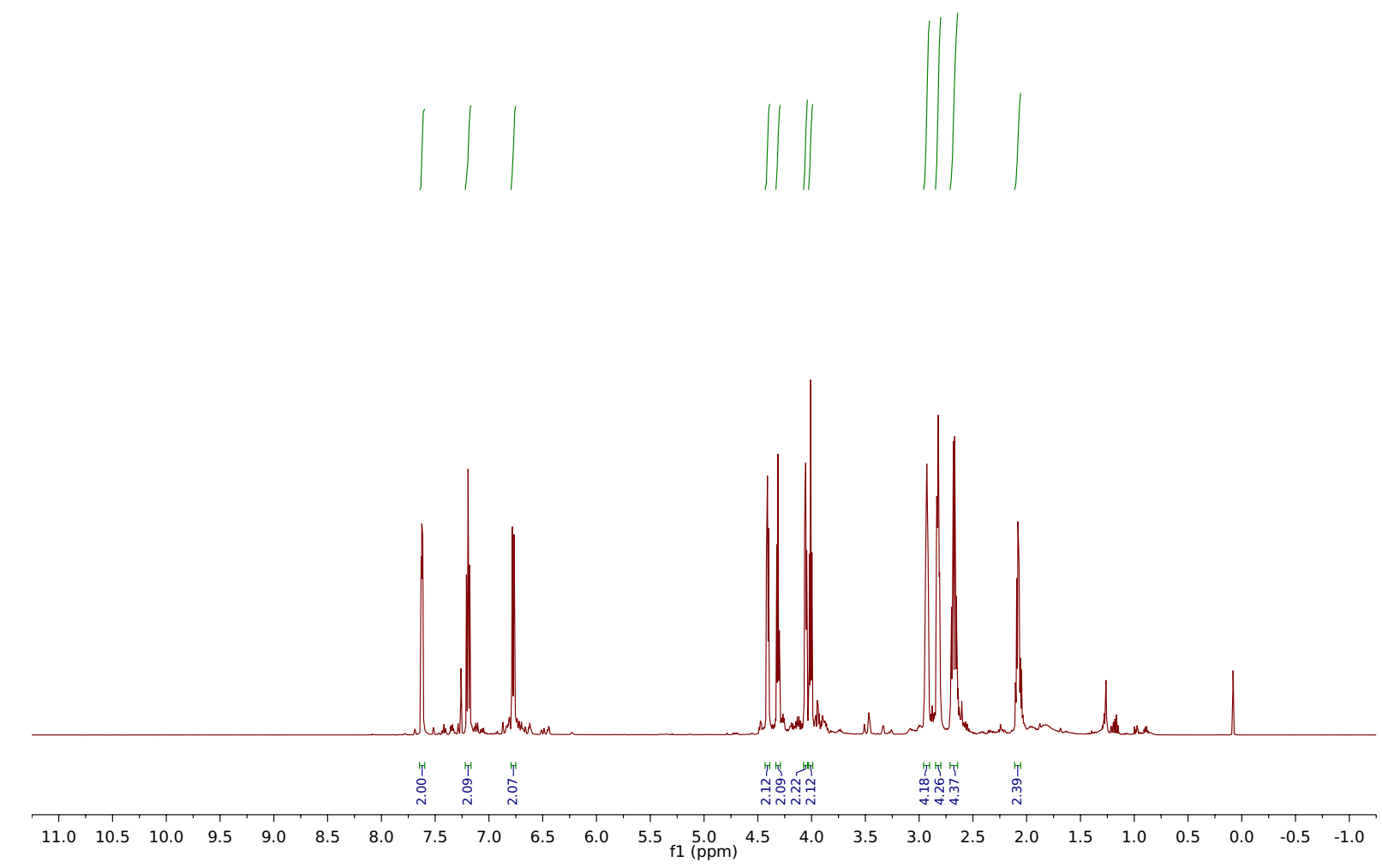

Figure S30. ${ }^{1} \mathrm{H}$ NMR spectrum of $\mathbf{S 8 .}$ 


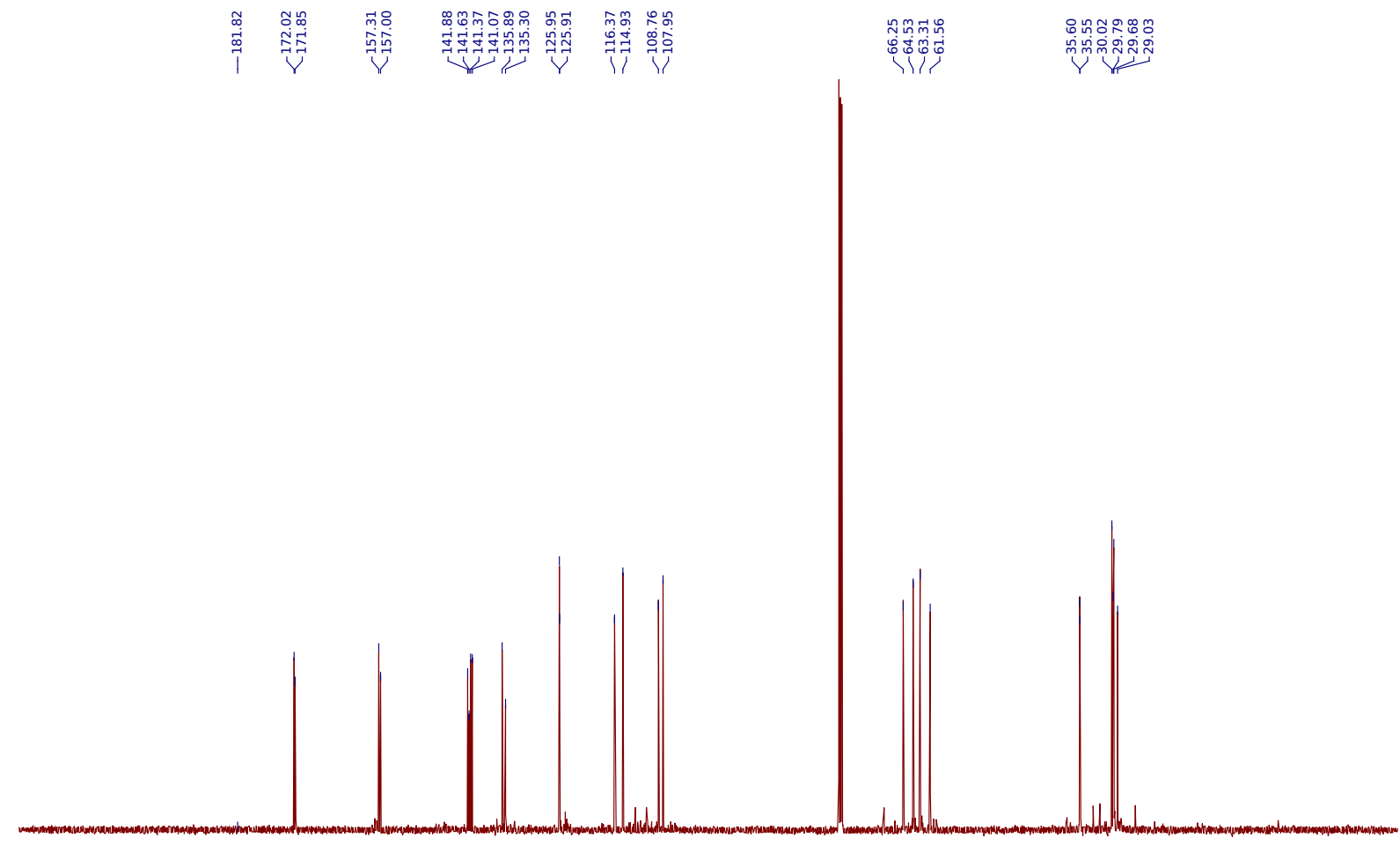

$\begin{array}{lllllllllllllllllllllllll}210 & 200 & 190 & 180 & 170 & 160 & 150 & 140 & 130 & 120 & 110 & 100 & 90 & 80 & 70 & 60 & 50 & 40 & 30 & 20 & 10 & 0 & -10\end{array}$

Figure S31. ${ }^{13} \mathrm{C}$ NMR spectrum of $\mathbf{S 8}$. 


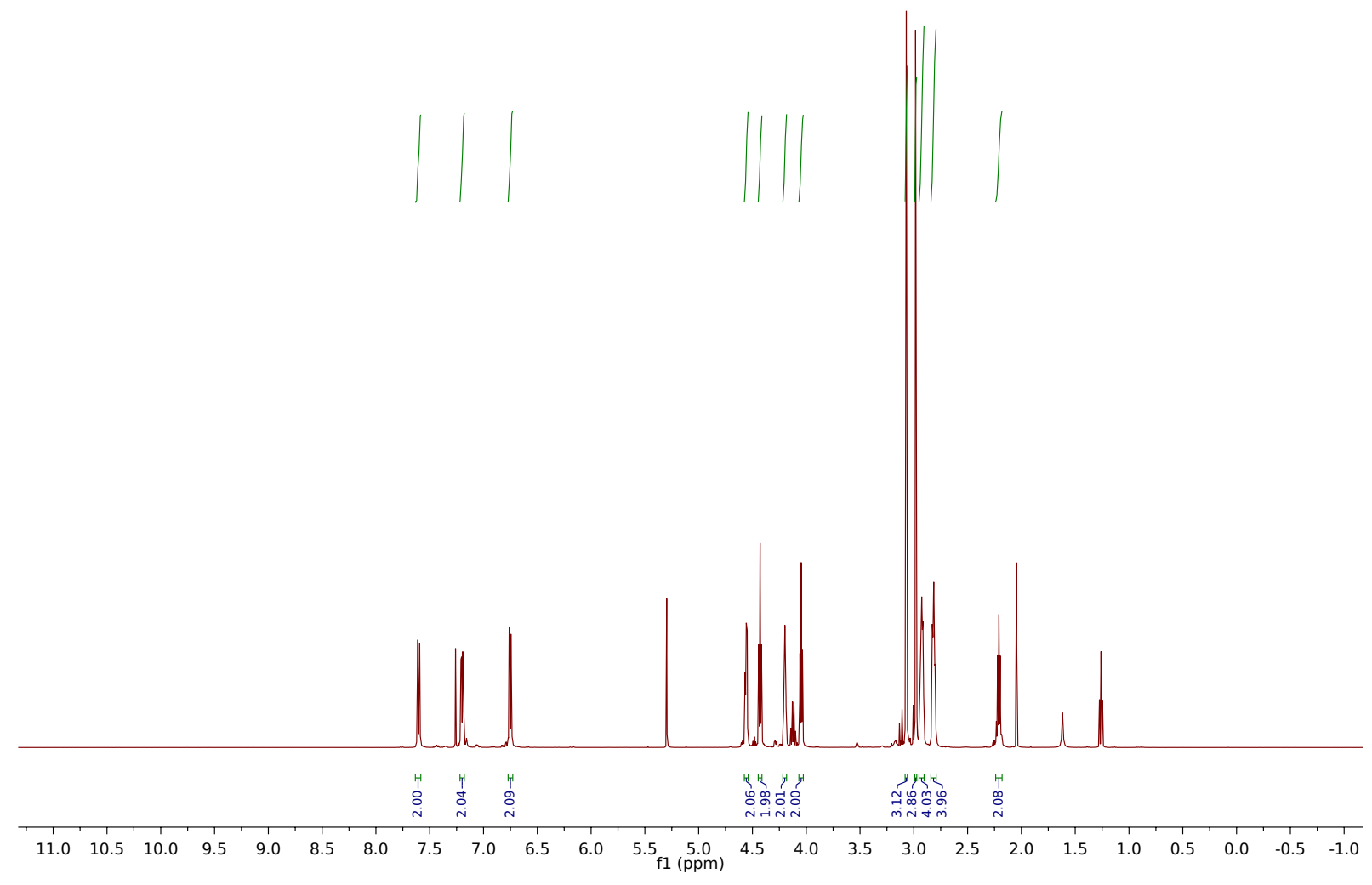

Figure S32. ${ }^{1} \mathrm{H}$ NMR spectrum of $\mathbf{S 1 0 .}$ 


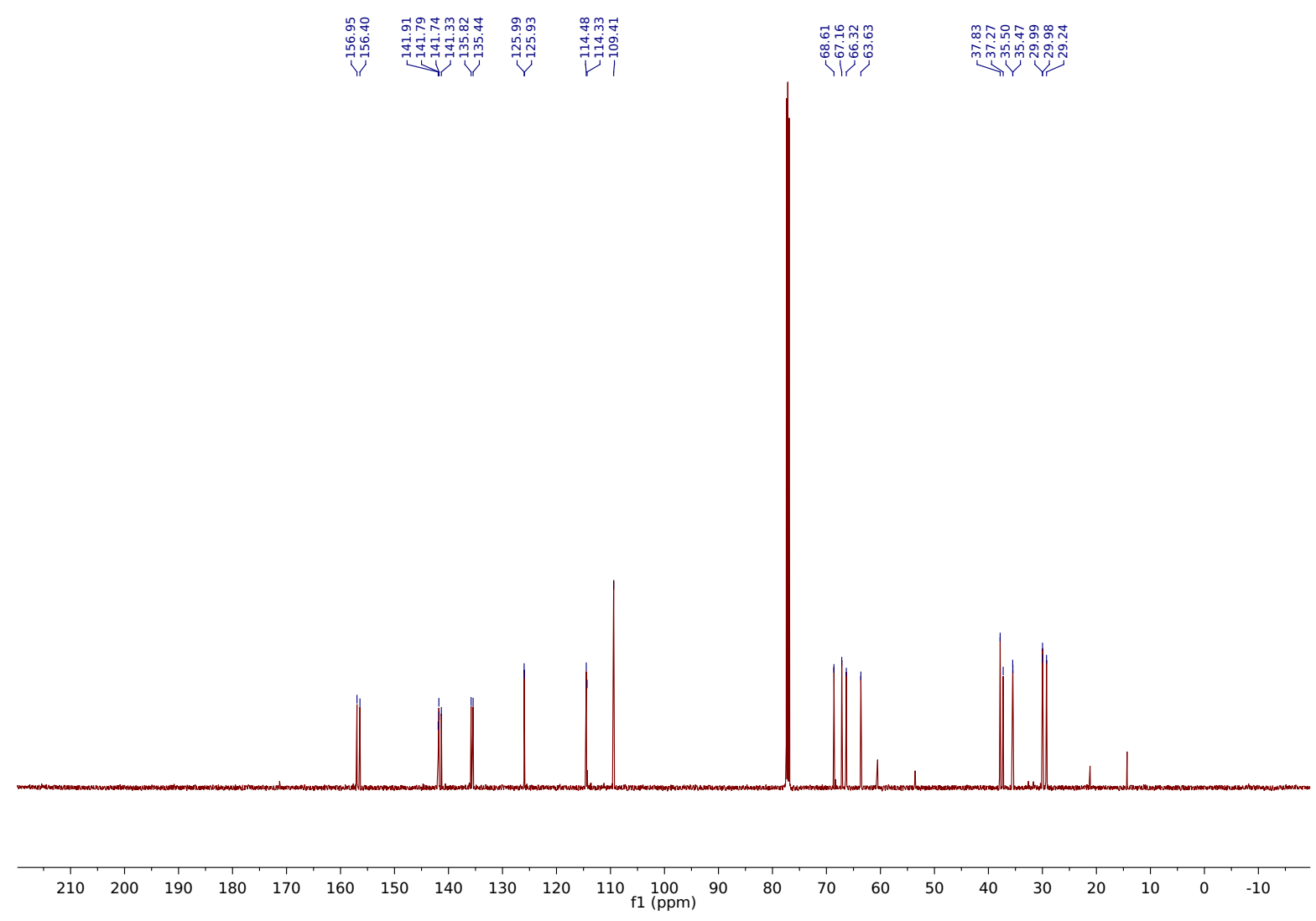

Figure S33. ${ }^{13} \mathrm{C}$ NMR spectrum of $\mathbf{S 1 0}$. 


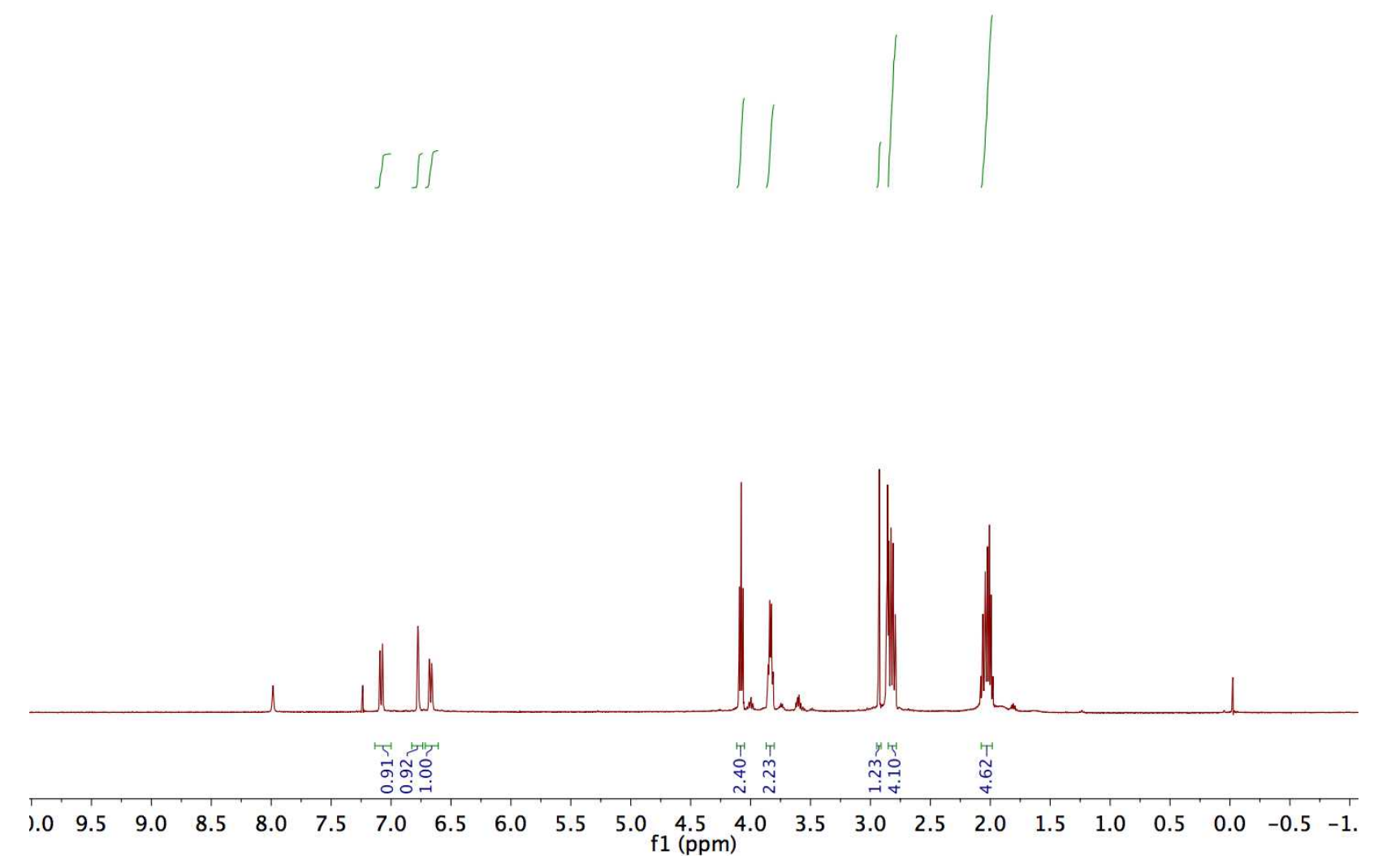

Figure S34. ${ }^{1} \mathrm{H}$ NMR spectrum of S11. 


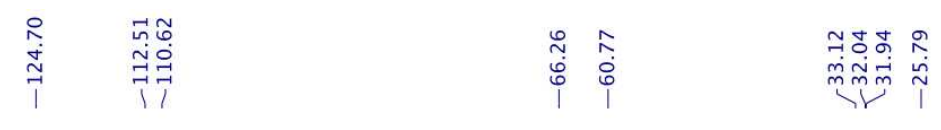

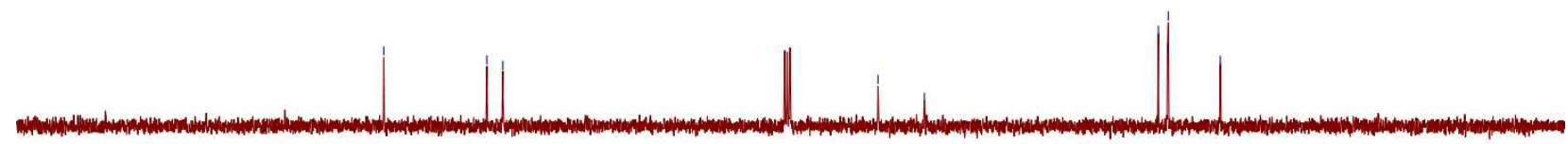

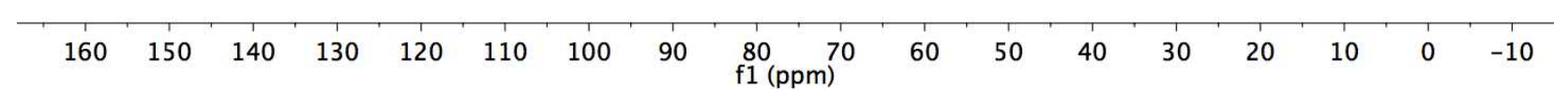

Figure S35. ${ }^{13} \mathrm{C}\left\{{ }^{1} \mathrm{H}\right\}$ NMR spectrum of $\mathbf{S} 11$. 


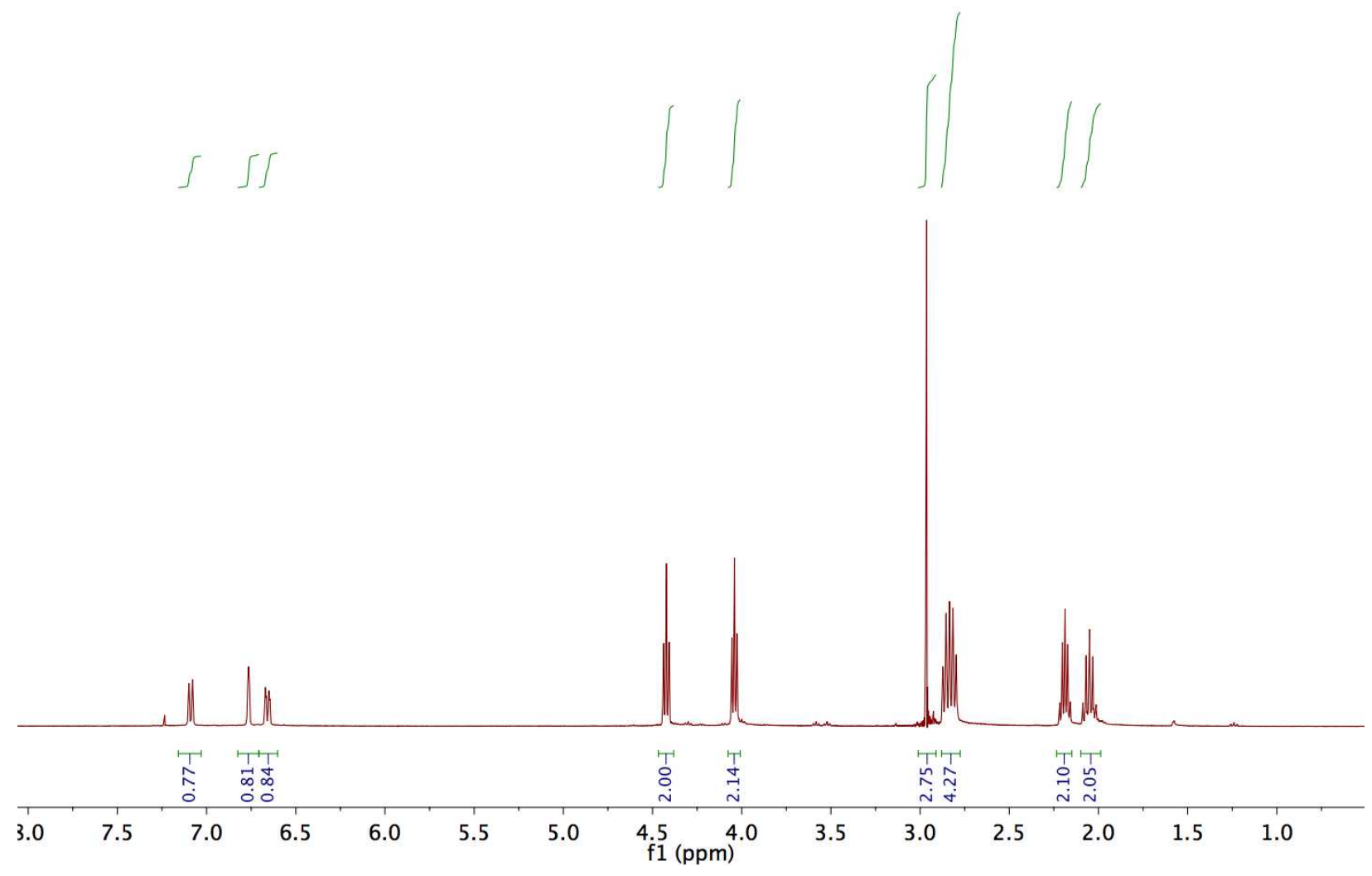

Figure S36. ${ }^{1} \mathrm{H}$ NMR spectrum of $\mathbf{S} 12$. 


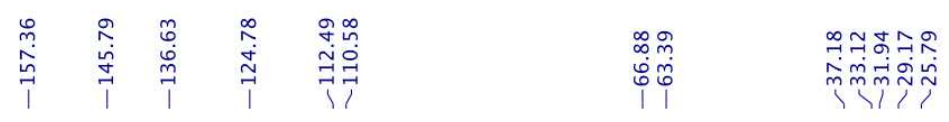

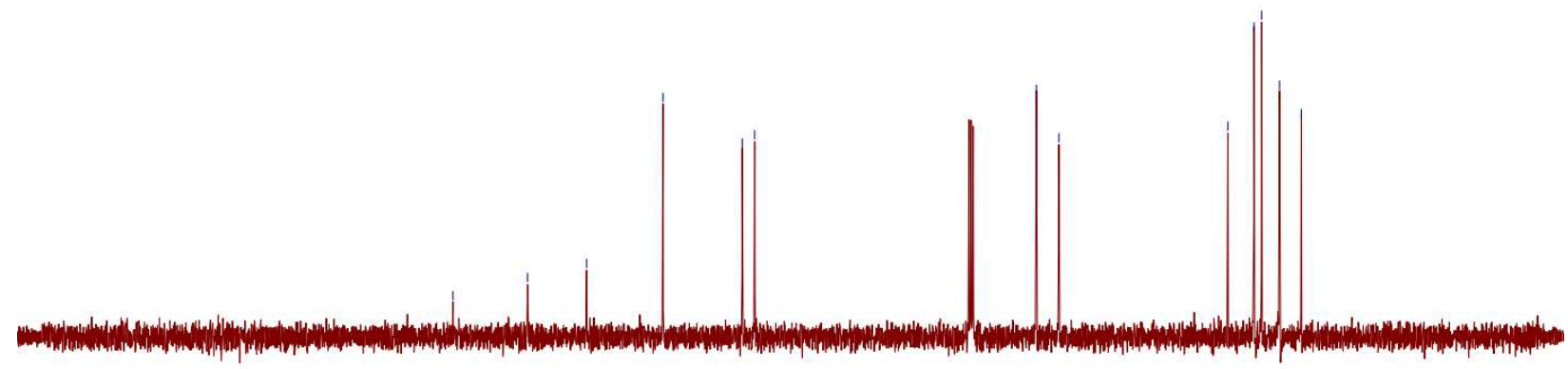

$\begin{array}{llllllllllllllllllllllllllllll}220 & 210 & 200 & 190 & 180 & 170 & 160 & 150 & 140 & 130 & 120 & 110 & 100 & 90 & 80 & 70 & 60 & 50 & 40 & 30 & 20 & 10 & 0 & -10\end{array}$

Figure S37. ${ }^{13} \mathrm{C}\left\{{ }^{1} \mathrm{H}\right\}$ NMR spectrum of $\mathbf{S 1 2}$. 


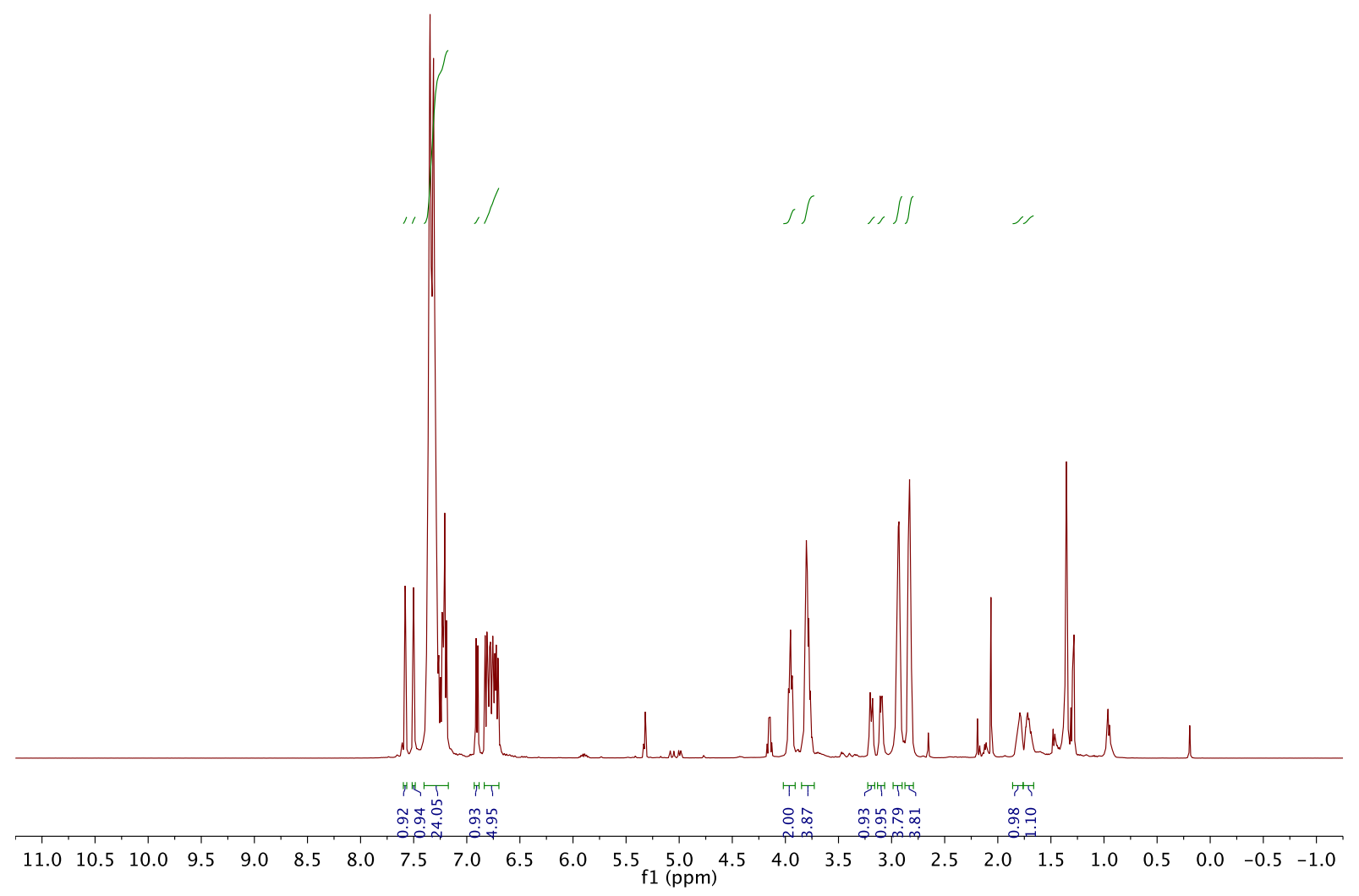

Figure S38. ${ }^{1} \mathrm{H}$ NMR spectrum of $Z(2,3)$. 


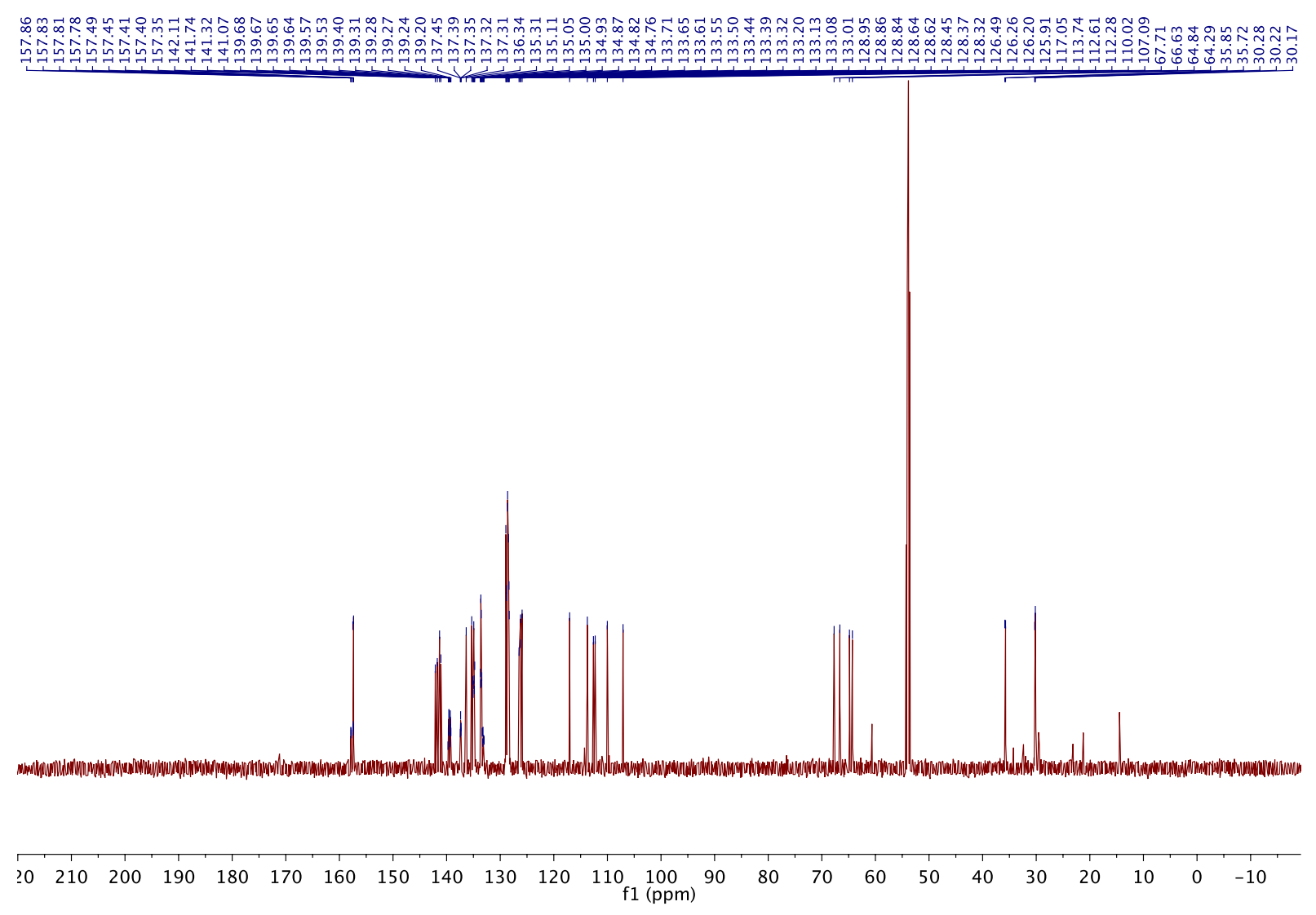

Figure S39. ${ }^{13} \mathrm{C}\left\{{ }^{1} \mathrm{H}\right\}$ NMR spectrum of $Z(2,3)$. 


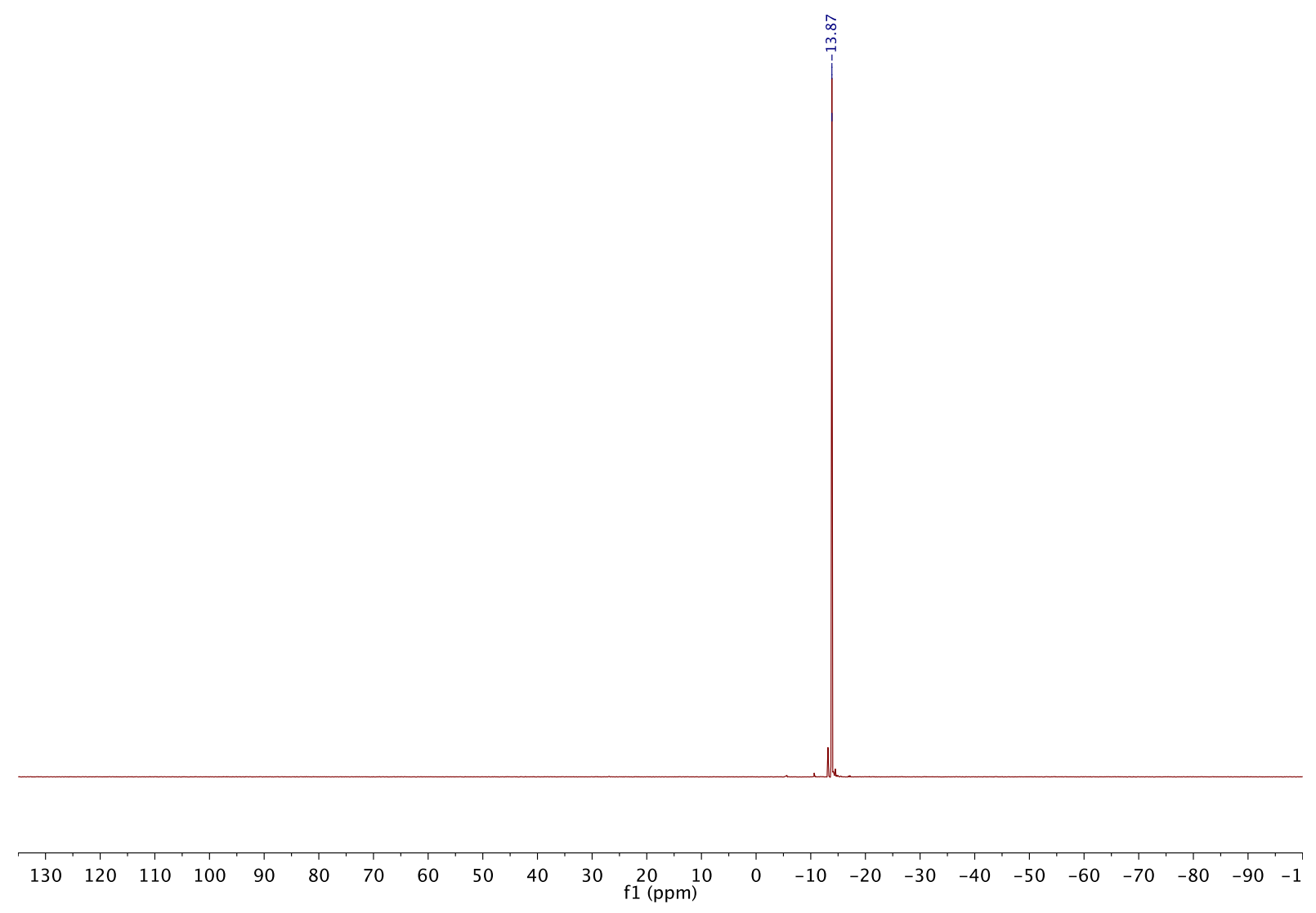

Figure S40. ${ }^{31} \mathrm{P}$ NMR spectrum of $Z(2,3)$. 


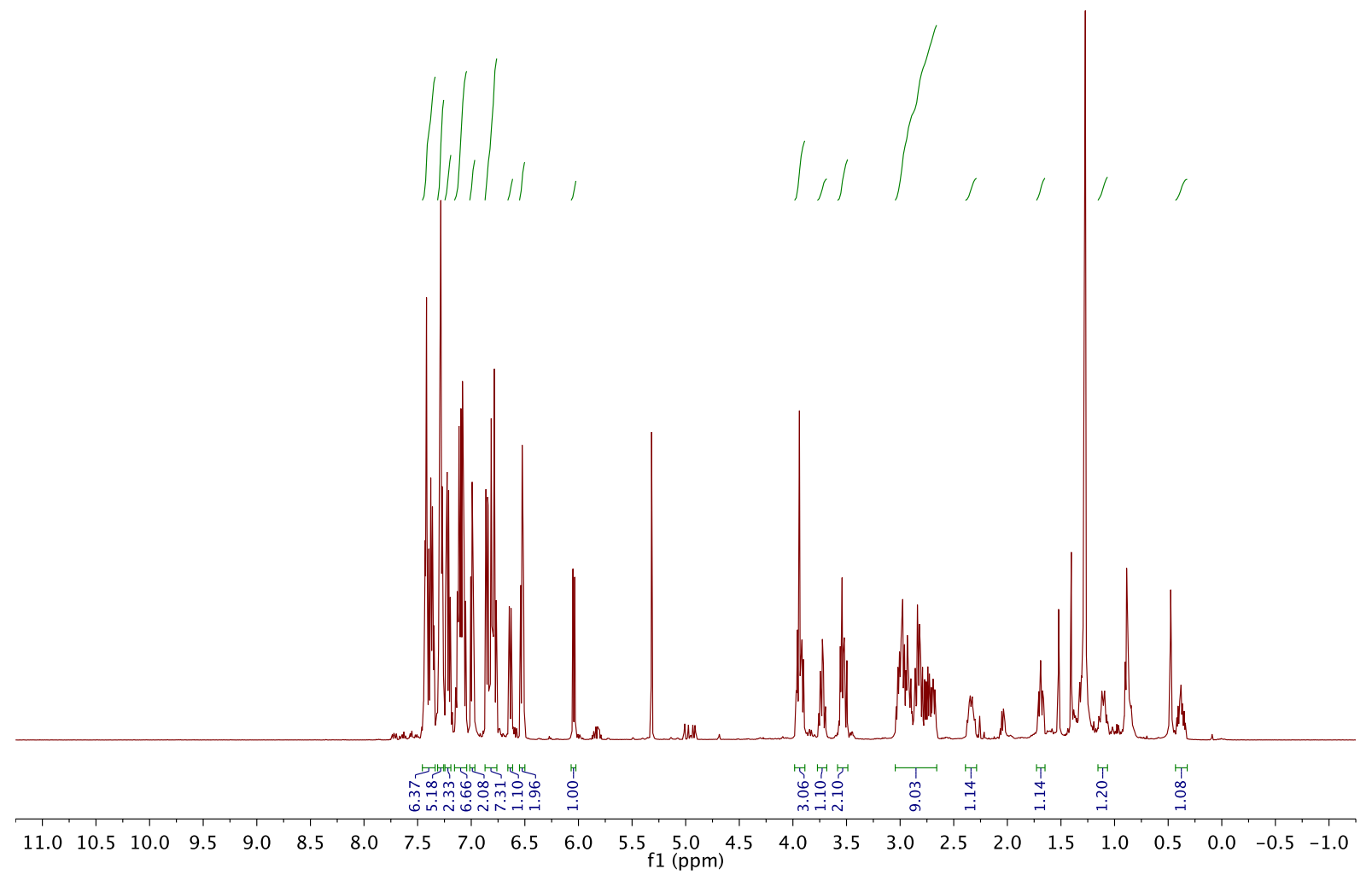

Figure S41. ${ }^{1} \mathrm{H}$ NMR spectrum of $E(2,3)$. 


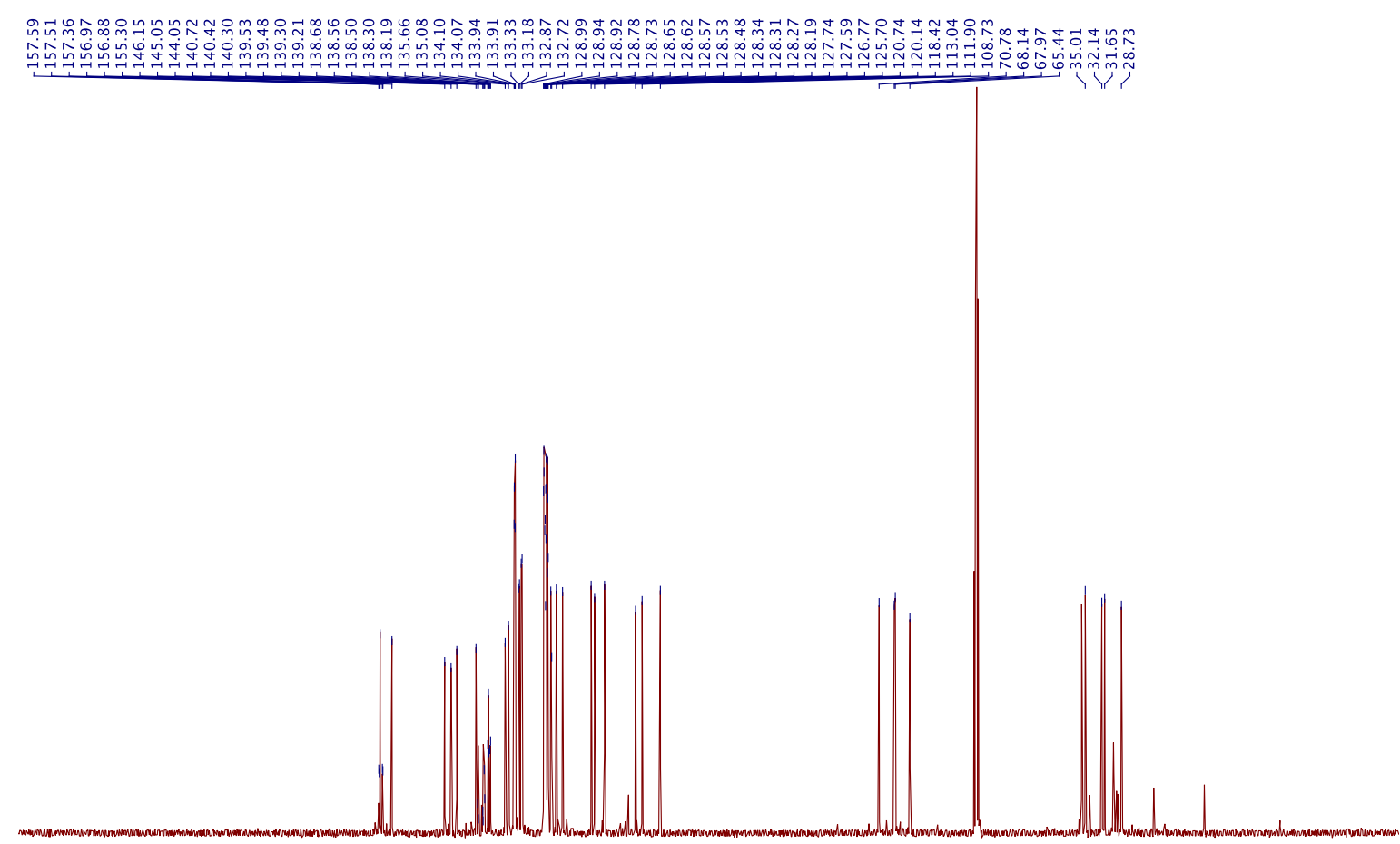

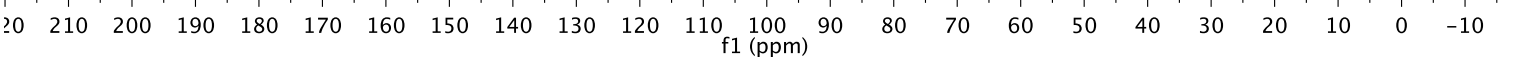

Figure S42. ${ }^{13} \mathrm{C}\left\{{ }^{1} \mathrm{H}\right\}$ NMR spectrum of $E(2,3)$. 


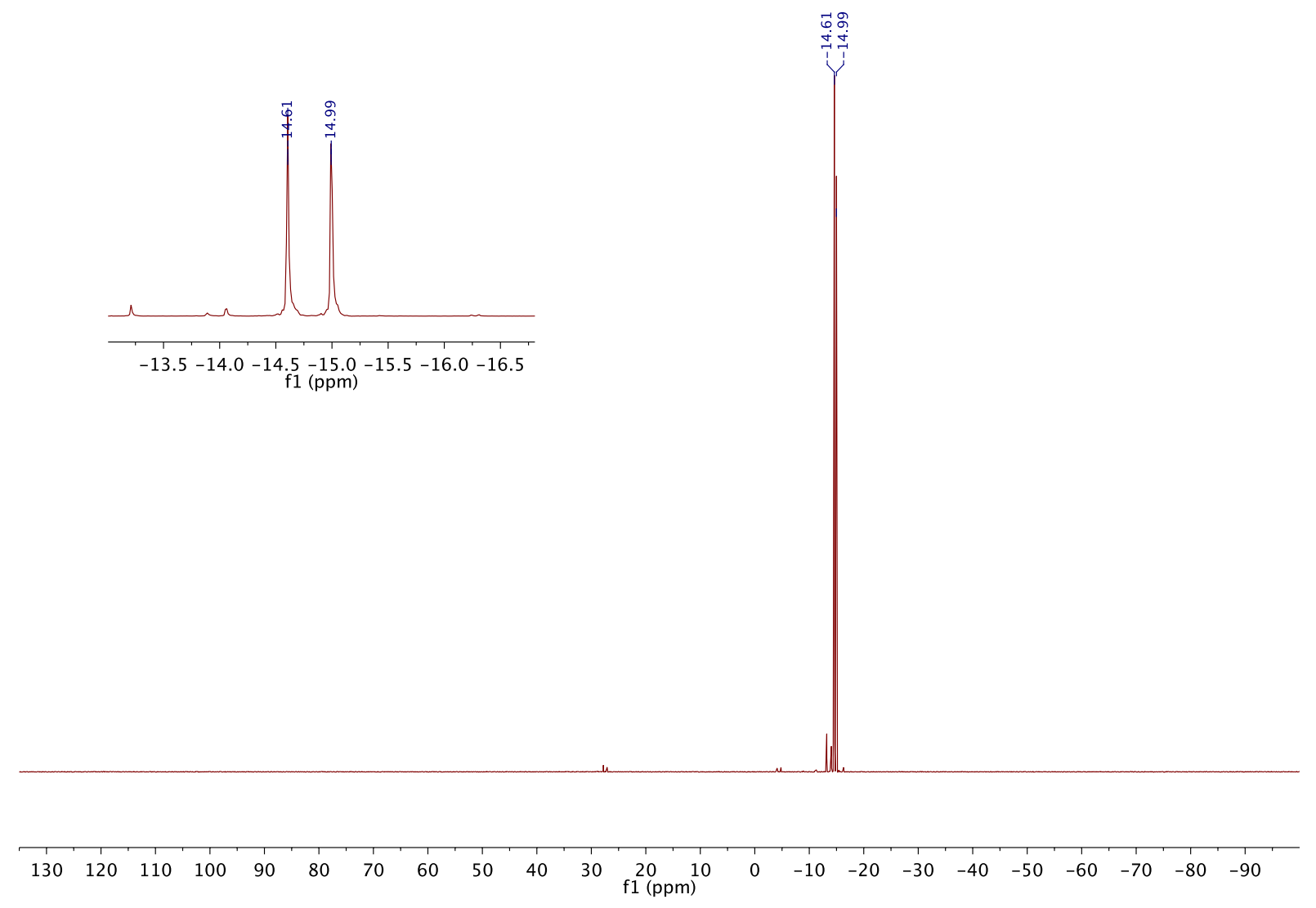

Figure S43. ${ }^{31} \mathrm{P}$ NMR spectrum of $E(2,3)$. 


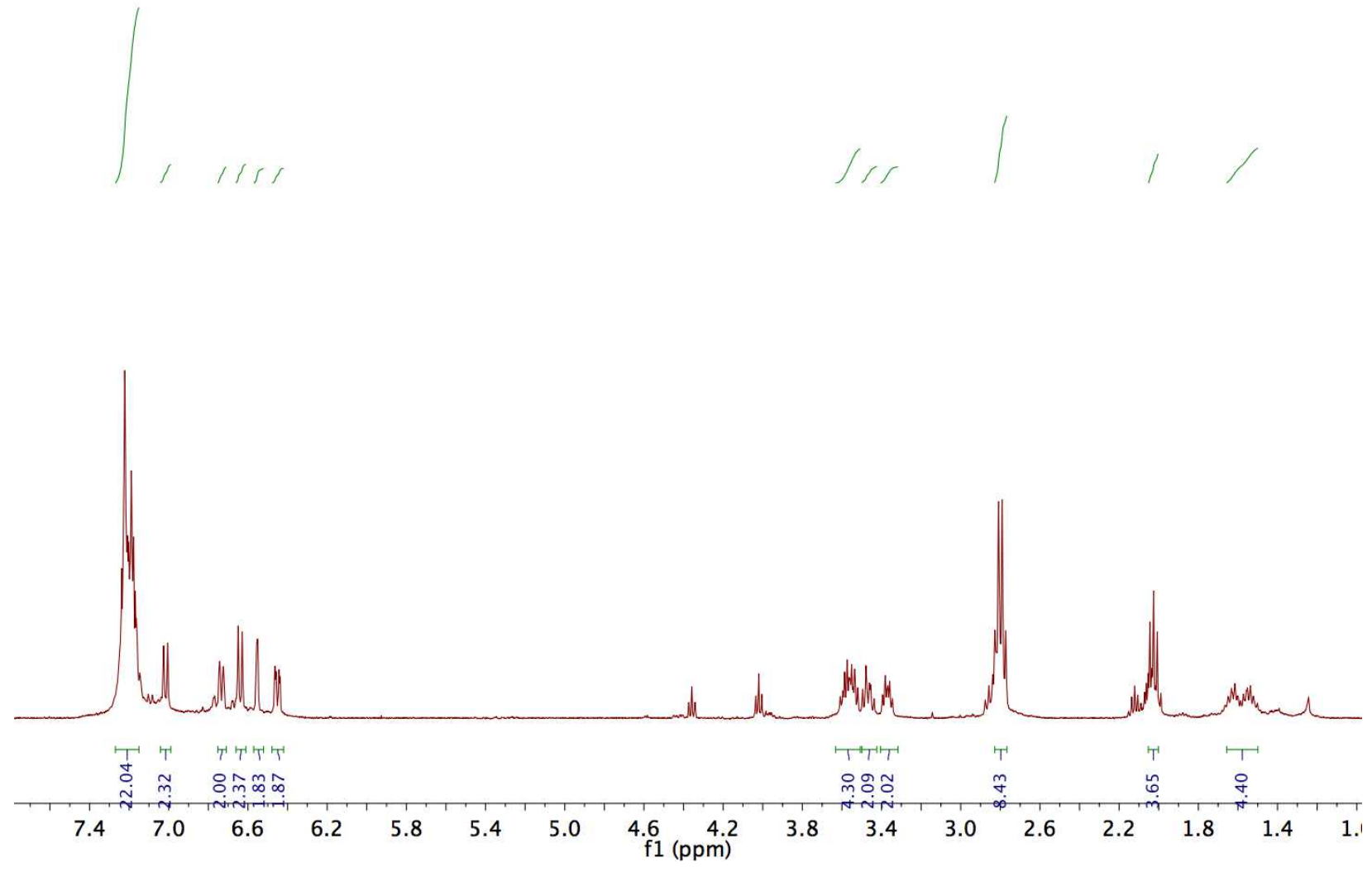

Figure S44. ${ }^{1} \mathrm{H}$ NMR spectrum of Indane-Biphep. 


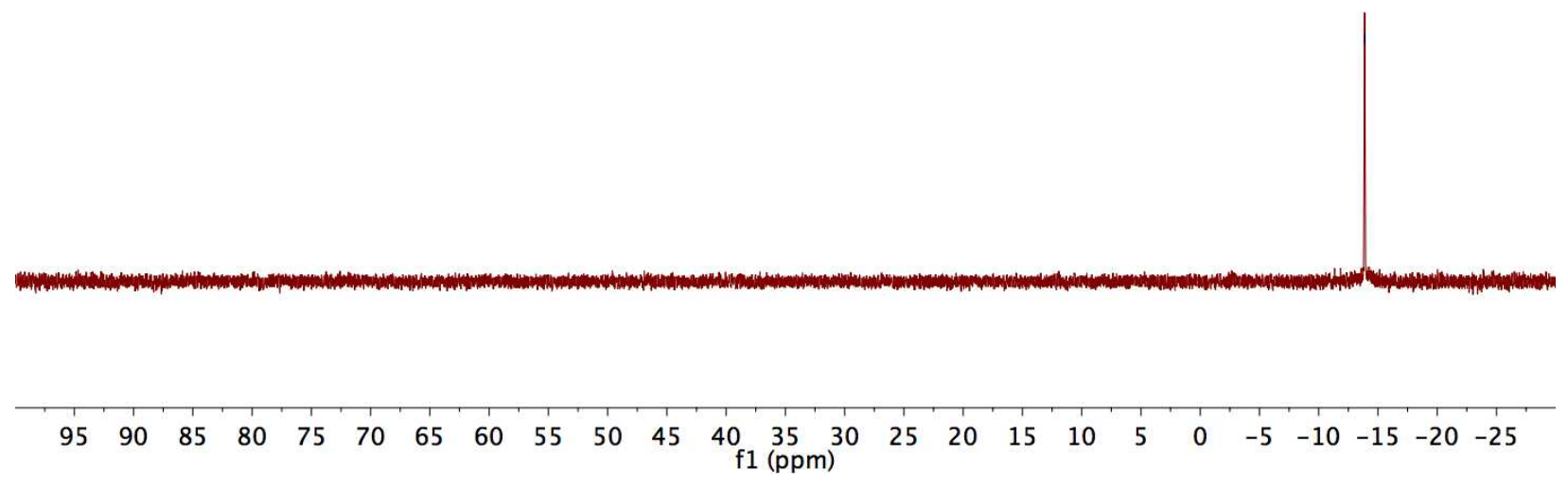

Figure S45. ${ }^{31} \mathrm{P}$ NMR spectrum of Indane-Biphep. 

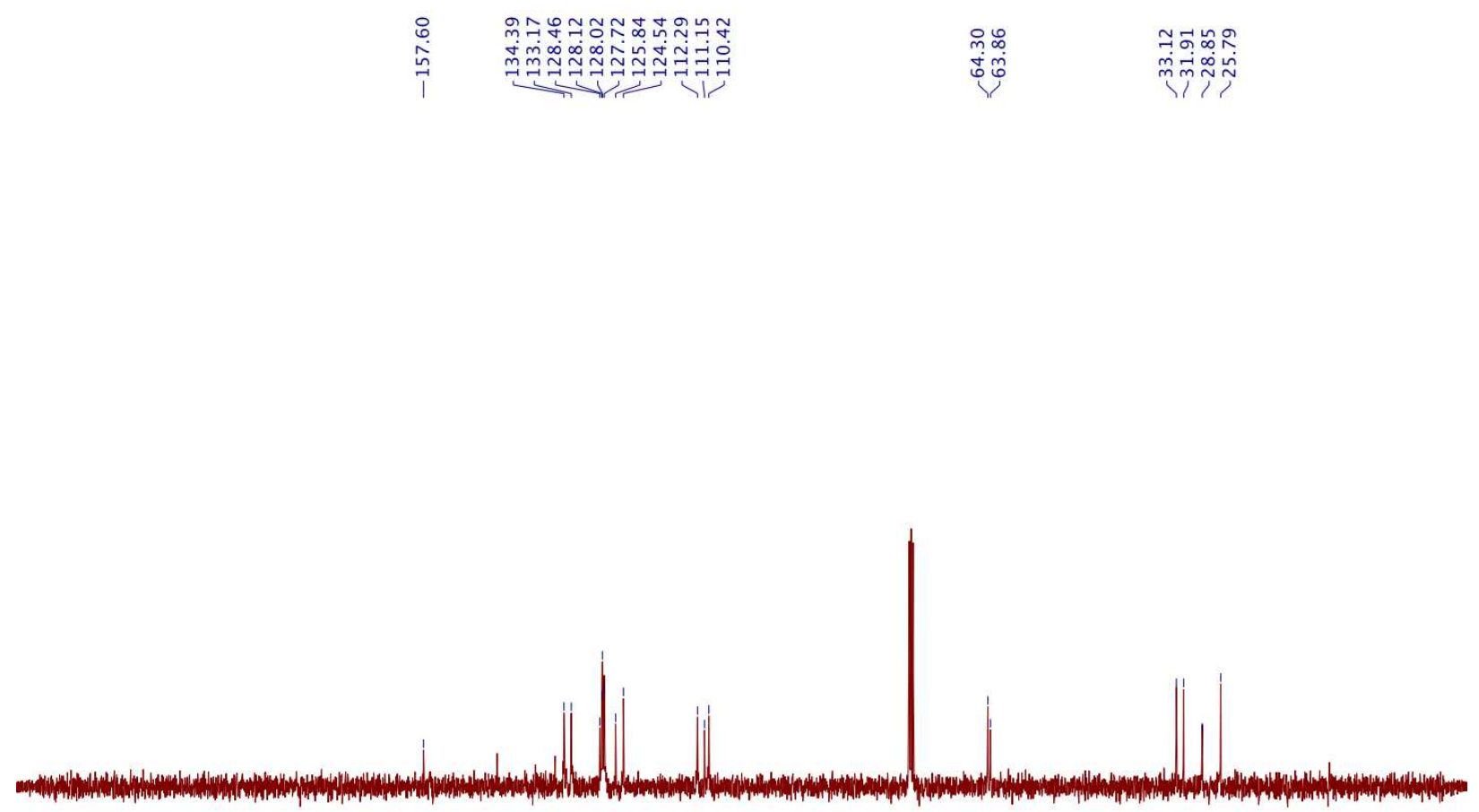

$\begin{array}{llllllllllllllllllllllll}220 & 210 & 200 & 190 & 180 & 170 & 160 & 150 & 140 & 130 & 120 & \begin{array}{l}110 \\ \mathrm{f} 1(\mathrm{ppm})\end{array} & 90 & 80 & 70 & 60 & 50 & 40 & 30 & 20 & 10 & 0 & -10\end{array}$

Figure S46. ${ }^{13} \mathrm{C}\left\{{ }^{1} \mathrm{H}\right\}$ NMR spectrum of Indane-Biphep. 


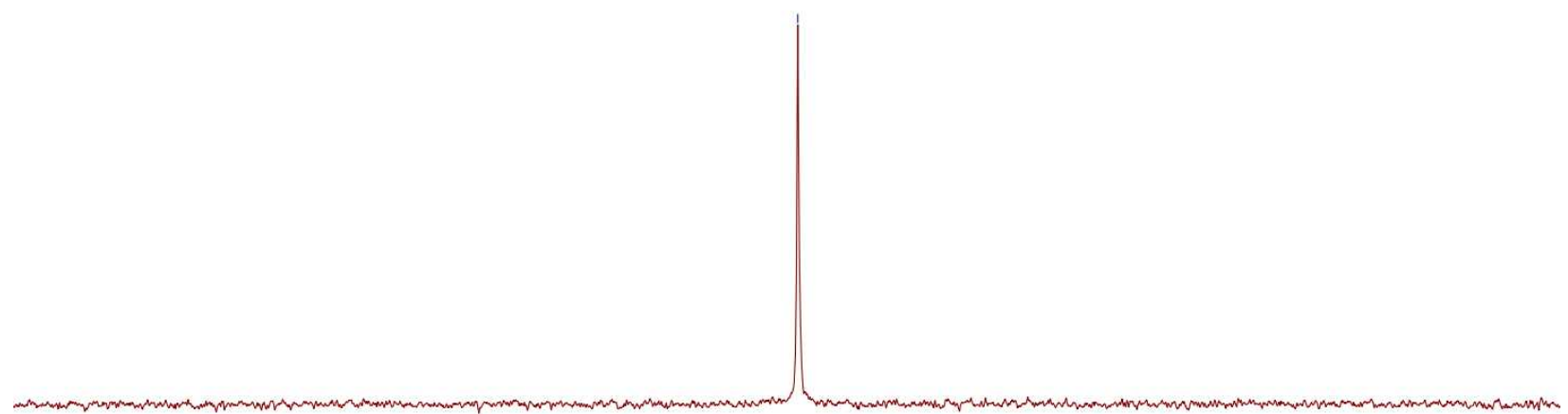

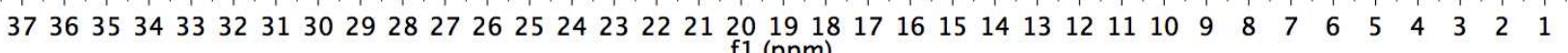
f1 (ppm)

Figure S47. ${ }^{31} \mathrm{P} N M R$ spectrum of $Z(3,3) \operatorname{Pd}(0)$. 


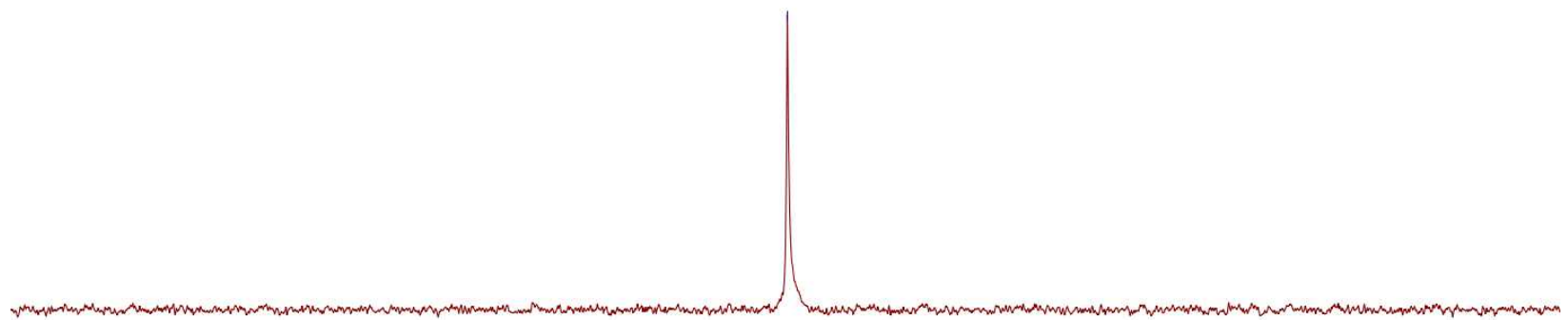

$10393837363534333231302928272625242322212019181716151413121110 \quad 9 \quad 8 \quad 7 \quad 6 \quad 5 \quad 4 \quad 3 \quad 2 \quad 1$

$$
\text { f1 (ppm) }
$$

Figure S48. ${ }^{31} \mathrm{P} N M R$ spectrum of $Z(2,2) \operatorname{Pd}(0)$. 


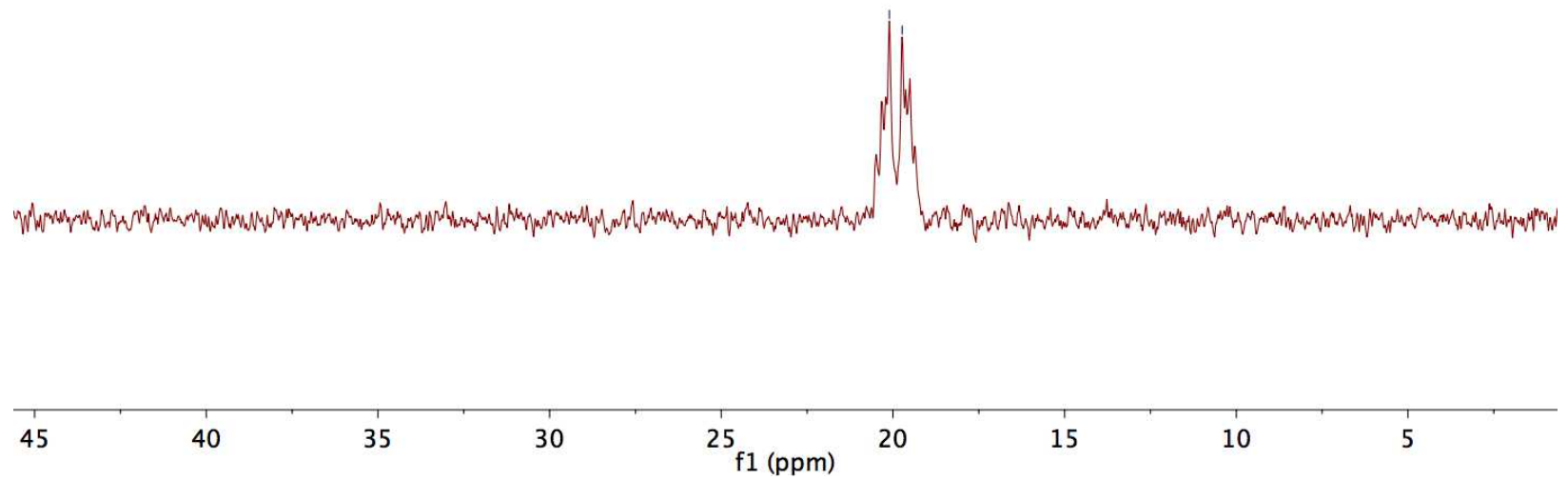

Figure S49. ${ }^{31} \mathrm{P}$ NMR spectrum of $Z(2,3) \mathrm{Pd}(0)$. 


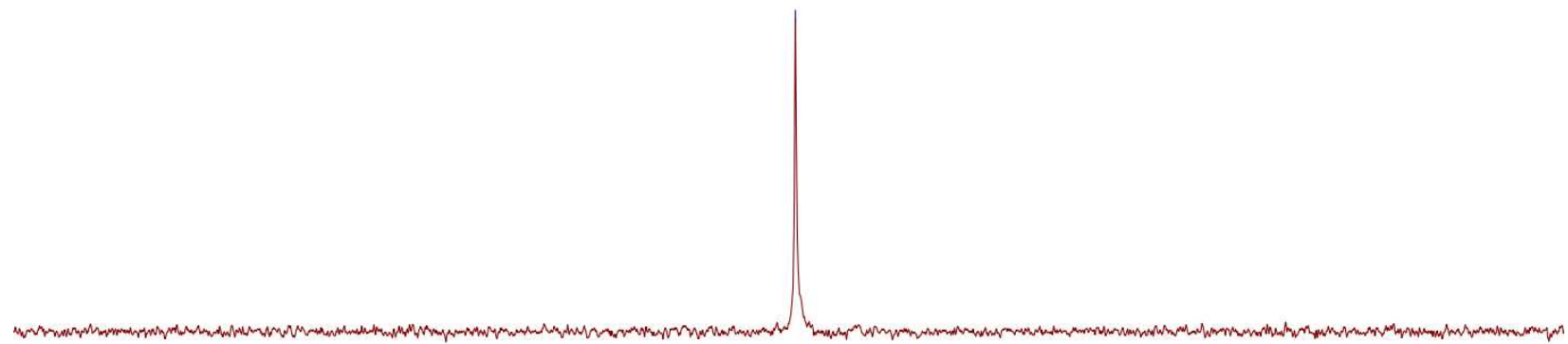

$0393837363534333231302928272625242322212019181716151413121110 \quad 9 \quad 8 \quad 7 \quad 6 \quad 5 \quad 4 \quad 3 \quad 2 \quad 1$ f1 (ppm)

Figure S50. ${ }^{31} \mathrm{P}$ NMR spectrum of (MeOBiphep) Pd(0). 


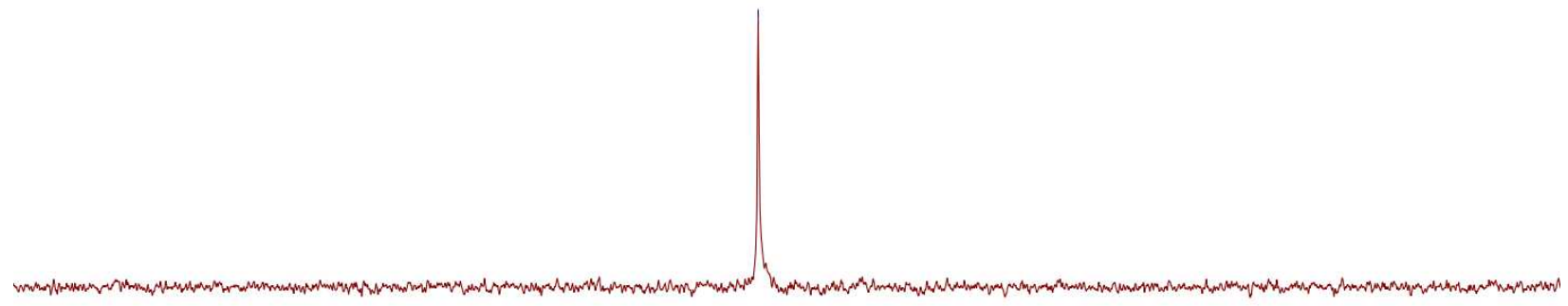

$4039383736353433323130292827262524232221201918171615141312111098^{7} \quad 6 \quad 5 \quad 4 \quad 3 \quad 2 \quad 1$ f1 (ppm)

Figure S51. ${ }^{31} \mathrm{P}$ NMR spectrum of $E(3,3) \operatorname{Pd}(0)$. 


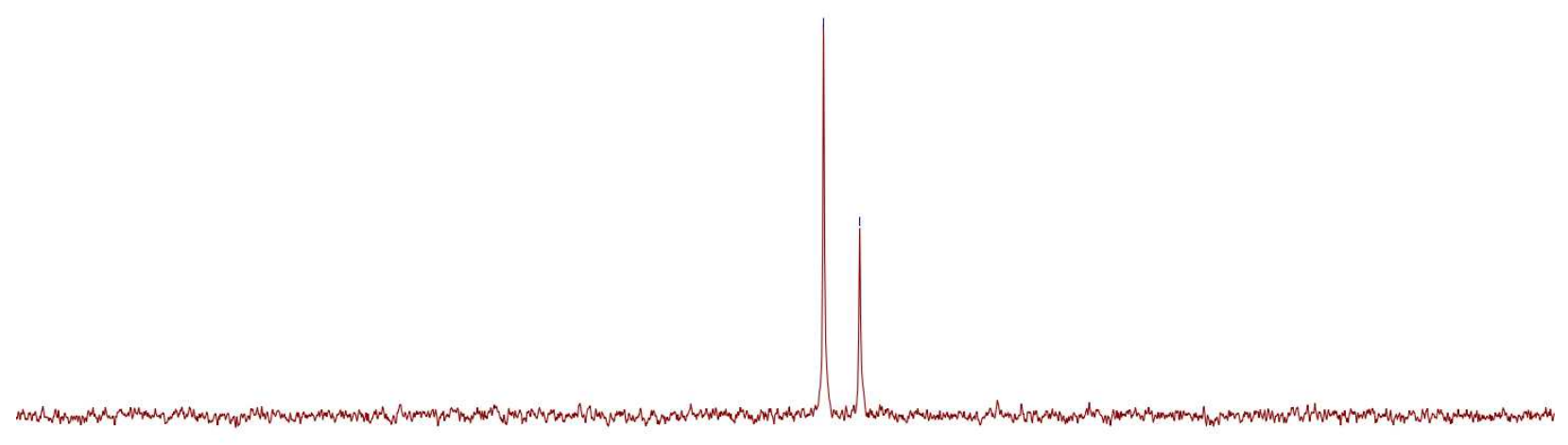

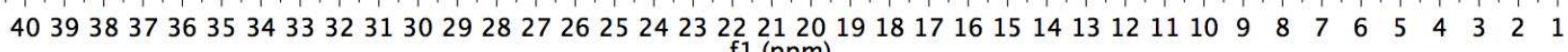
$\mathrm{f} 1(\mathrm{ppm})$

Figure S52. ${ }^{31} \mathrm{P}$ NMR spectrum of (Indane-Biphep) $\mathrm{Pd}(0)$. 
$\mathrm{Z}(2,2 \mathrm{C}) \mathrm{PdCl} 2 \mathrm{H}$

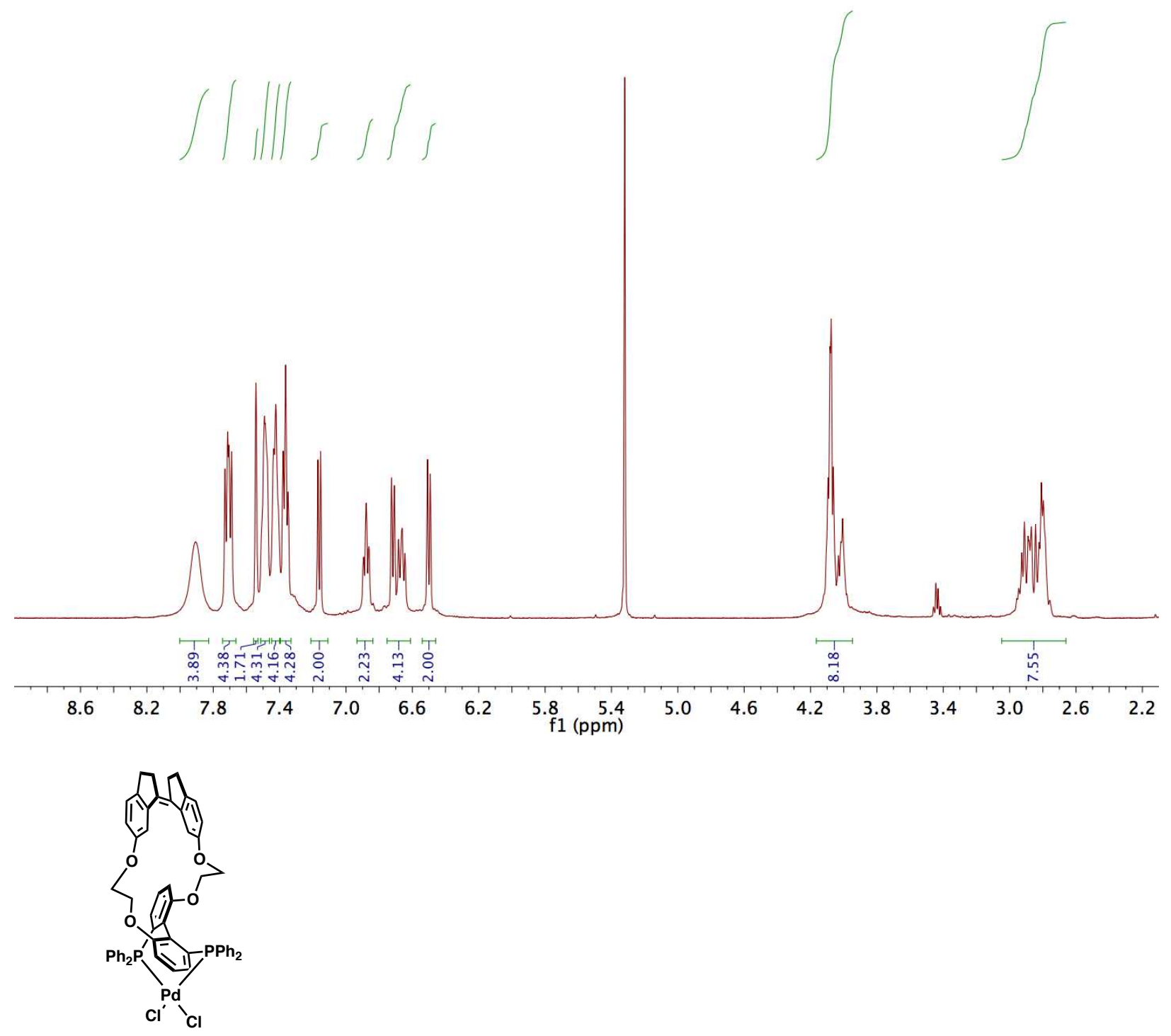

Figure S53. ${ }^{1} \mathrm{H}$ NMR spectrum of $Z(2,2) \mathrm{PdCl}_{2}$. 
$\mathrm{Z}(2,2 \mathrm{C}) \mathrm{PdCl} 2_{-} \mathrm{P}$

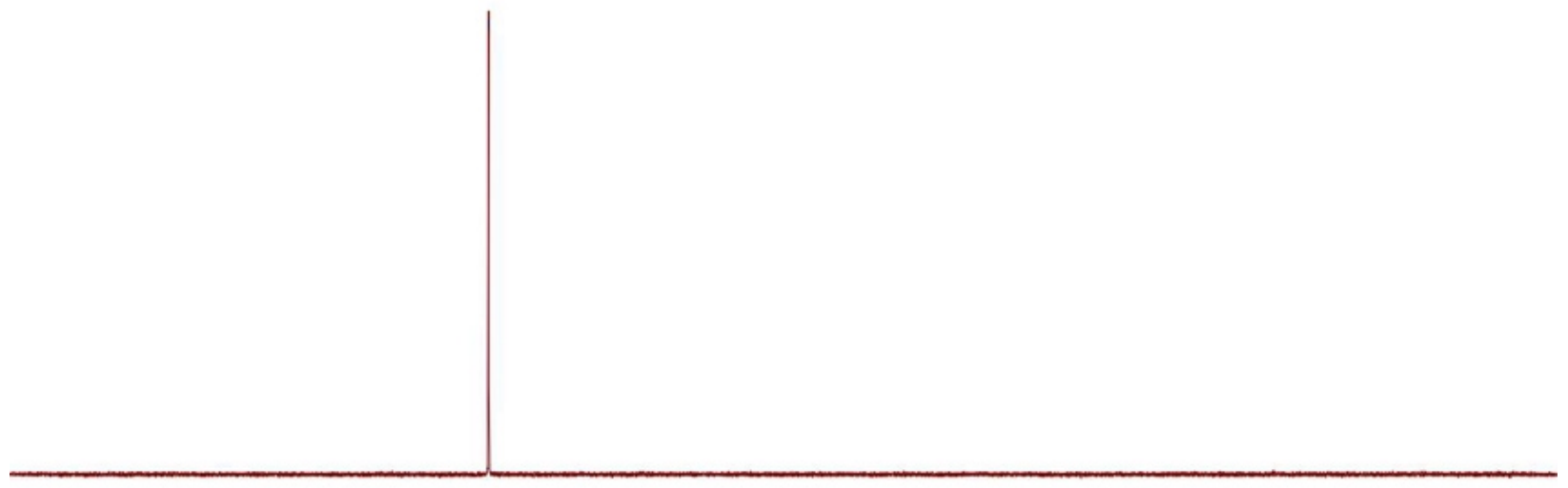

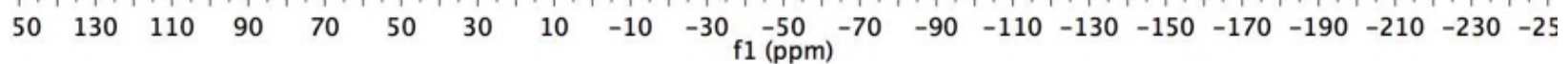

Figure S54. ${ }^{31} \mathrm{P}$ NMR spectrum of $Z(2,2) \mathrm{PdCl}_{2}$. 


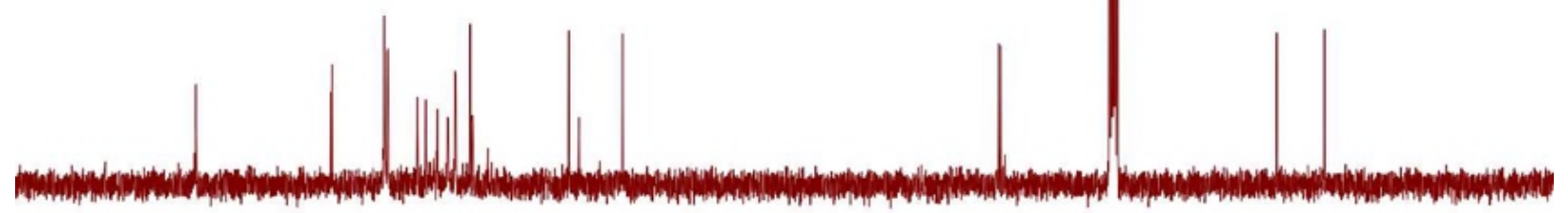

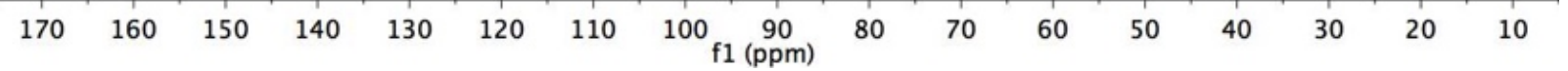

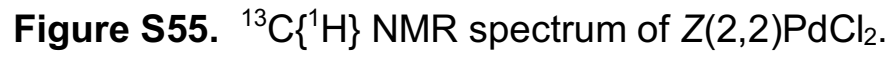


$\mathrm{Z}(2,3 \mathrm{C}) \mathrm{PdCl} 2 \mathrm{H}$
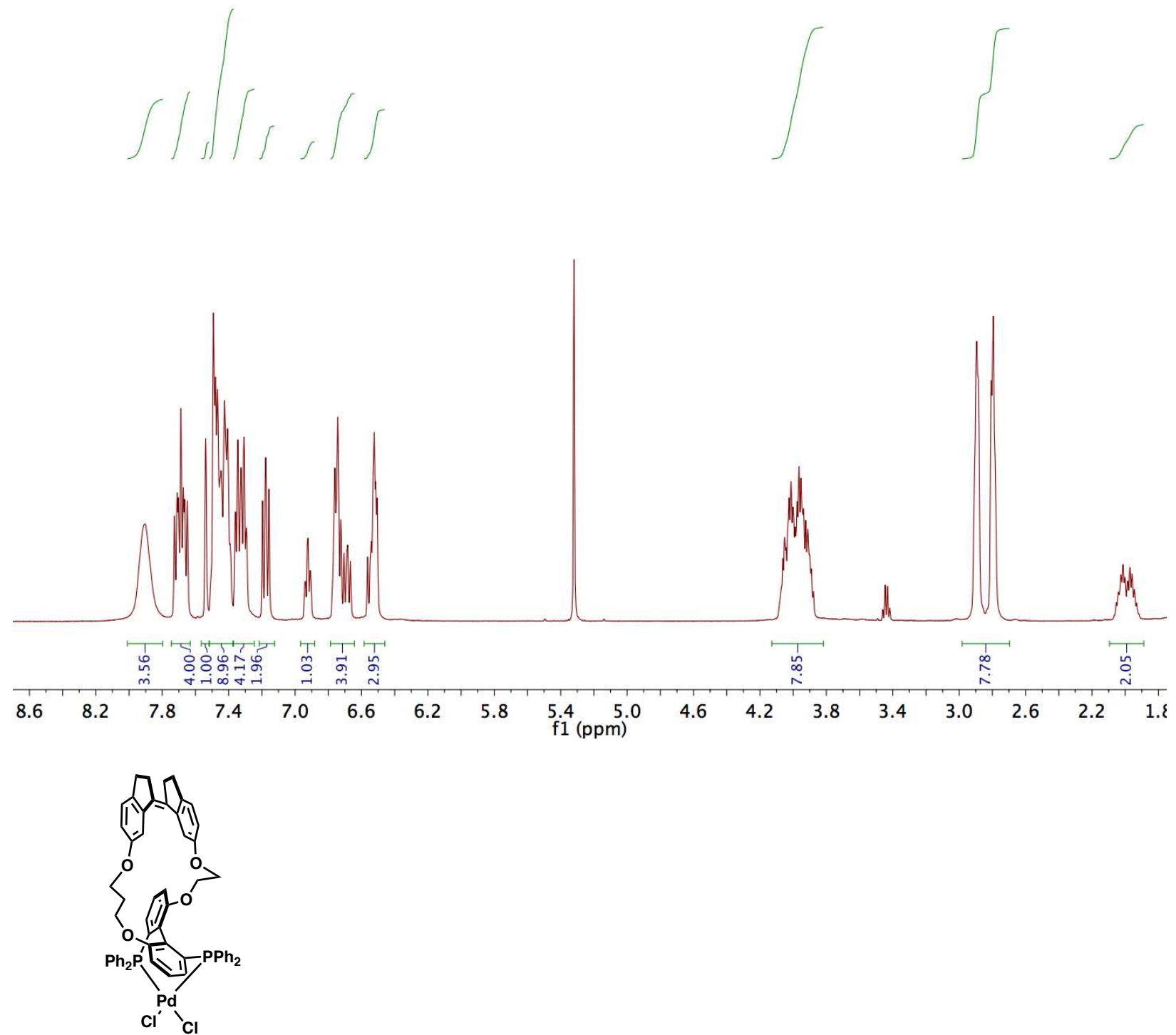

Figure S56. ${ }^{1} \mathrm{H}$ NMR spectrum of $Z(2,3) \mathrm{PdCl}_{2}$. 
$\mathrm{Z}(2,3 \mathrm{C}) \mathrm{PdCl} 2 \mathrm{P}$

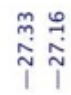

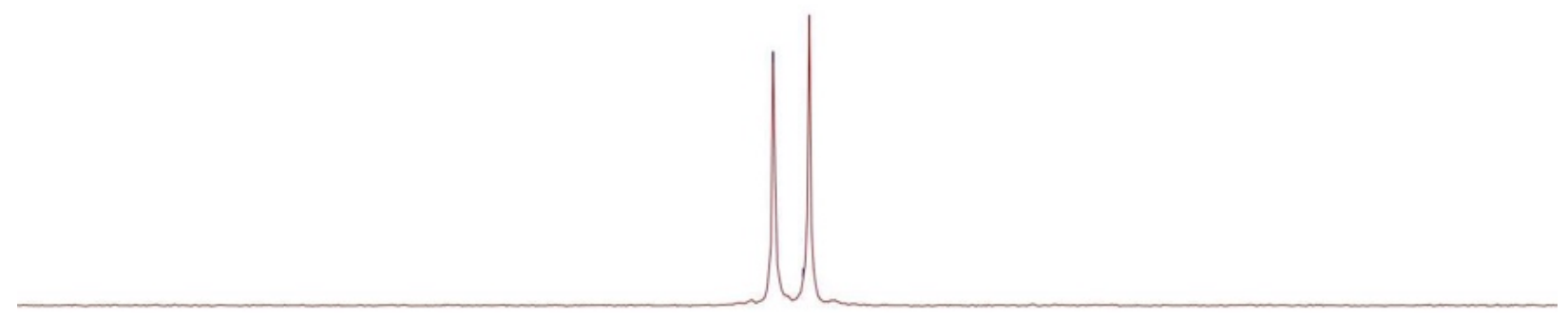

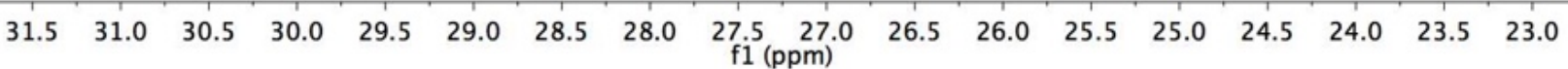

Figure S57. ${ }^{31} \mathrm{P}$ NMR spectrum of $Z(2,3) \mathrm{PdCl}_{2}$. 


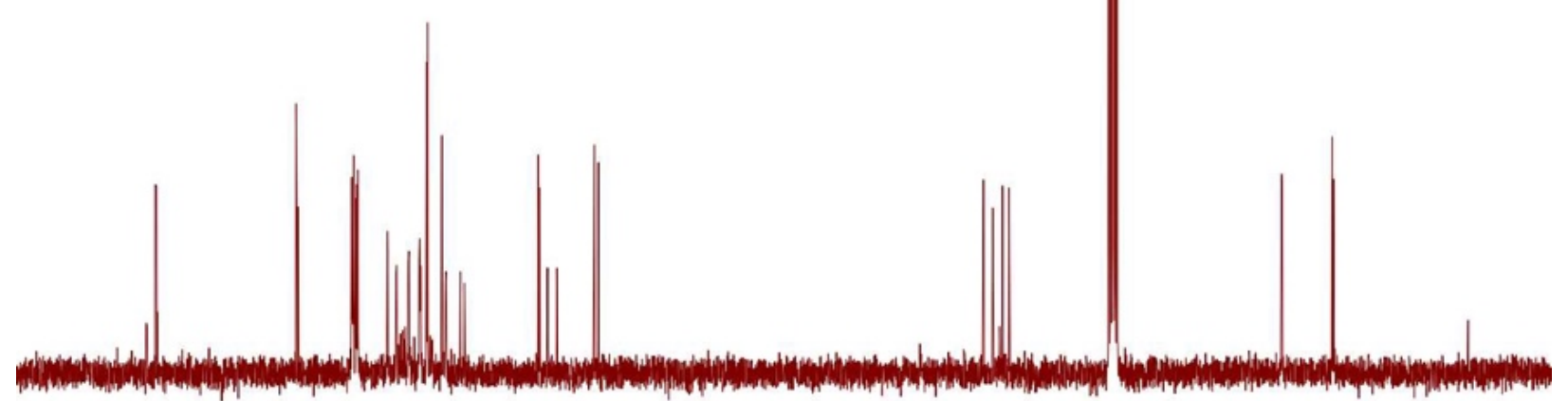

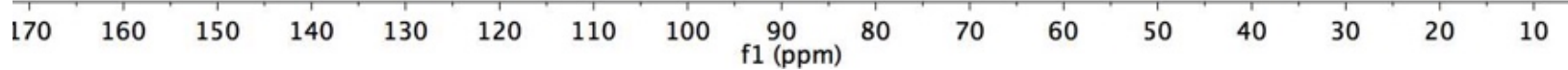

Figure S58. ${ }^{13} \mathrm{C}\left\{{ }^{1} \mathrm{H}\right\}$ NMR spectrum of $Z(2,3) \mathrm{PdCl}_{2}$. 
Z(3,3C)PdCl2_H

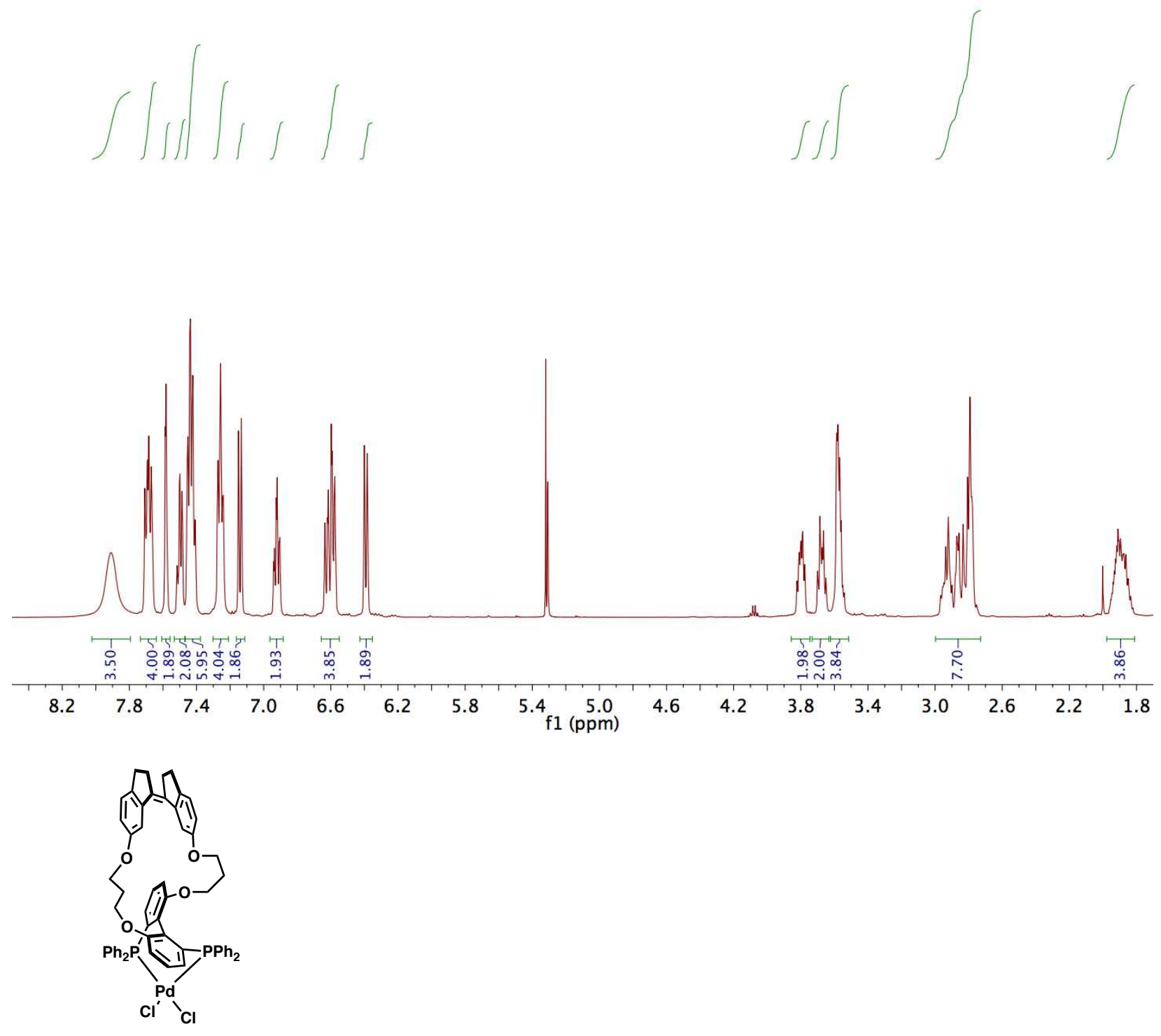

Figure S59. ${ }^{1} \mathrm{H}$ NMR spectrum of $Z(3,3) \mathrm{PdCl}_{2}$. 
$\mathrm{Z}(3,3 \mathrm{C}) \mathrm{PdCl}$ _P

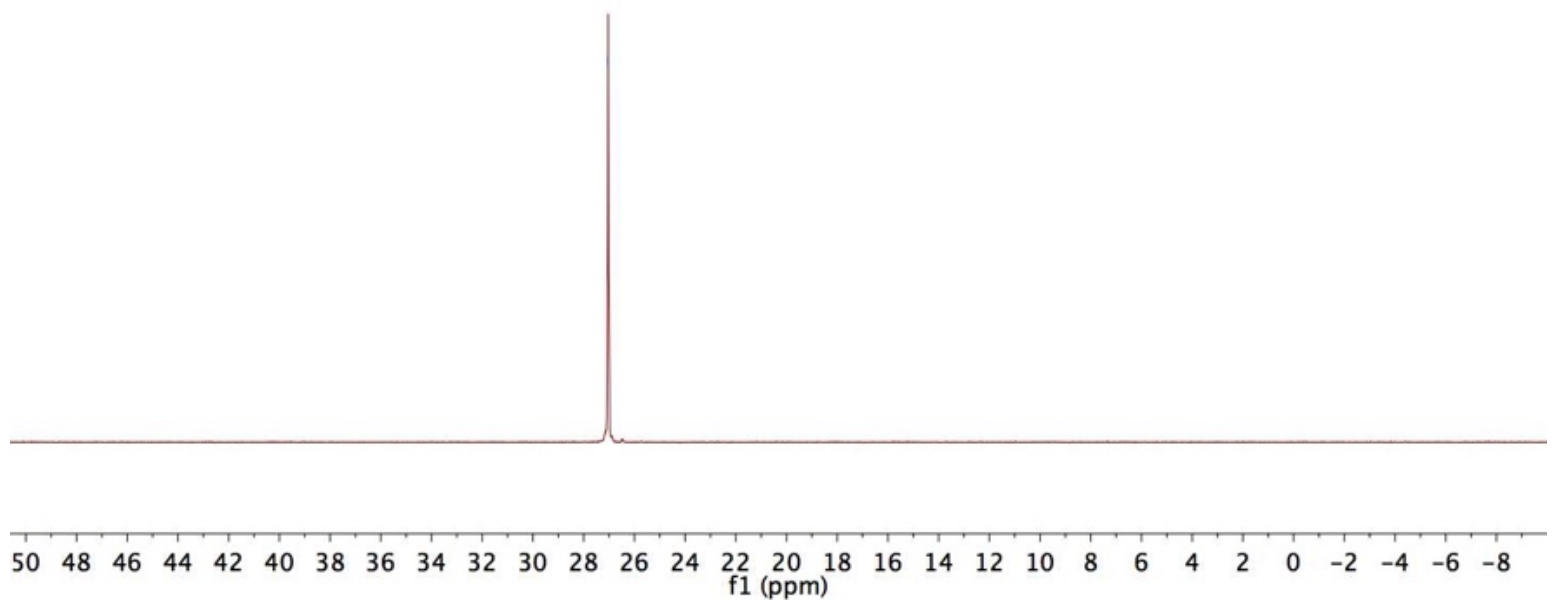

Figure S60. ${ }^{31} \mathrm{P}$ NMR spectrum of $Z(3,3) \mathrm{PdCl}_{2}$. 


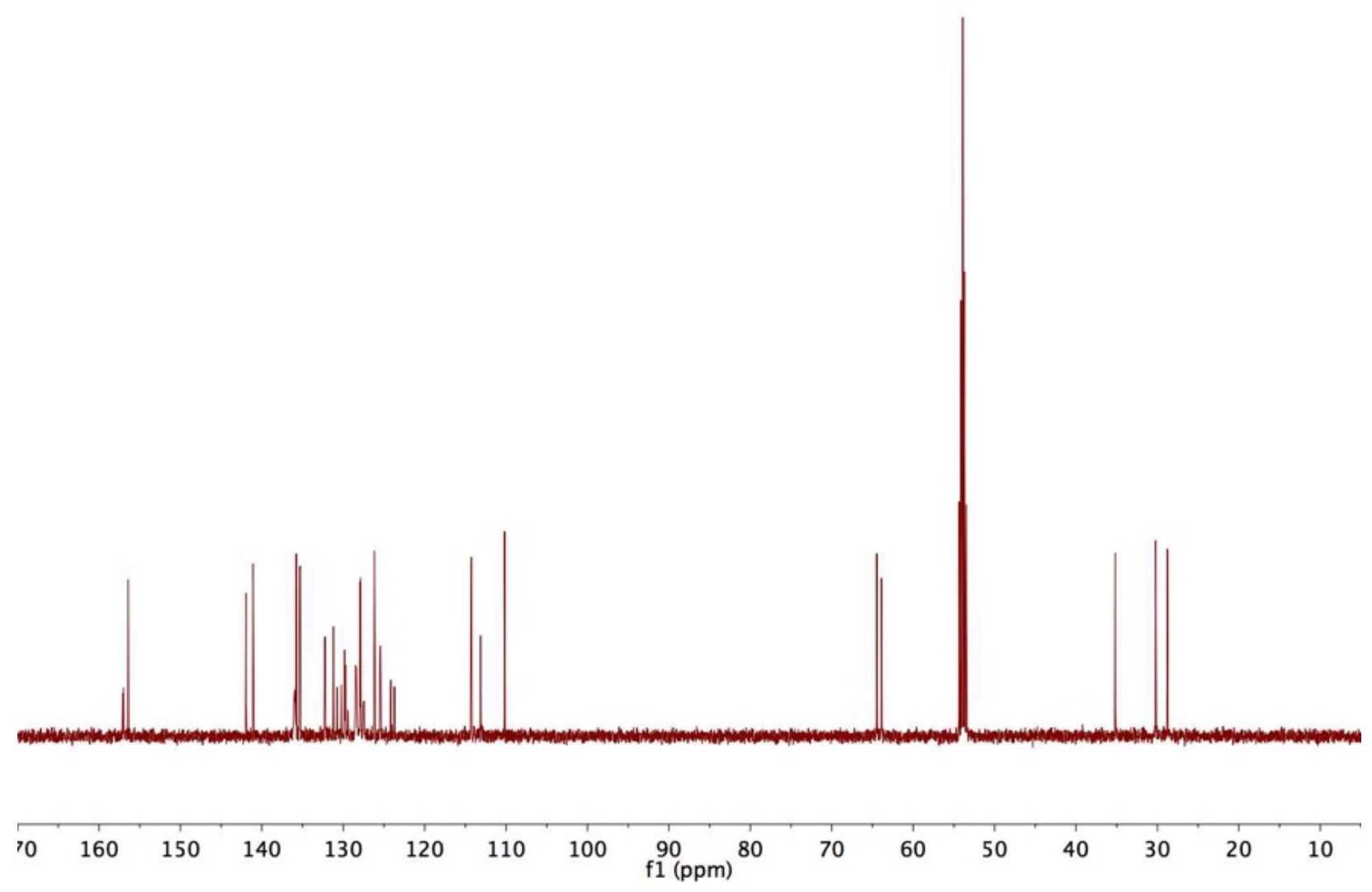

Figure S61. ${ }^{13} \mathrm{C}\left\{{ }^{1} \mathrm{H}\right\}$ NMR spectrum of $Z(3,3) \mathrm{PdCl}_{2}$. 
$\mathrm{E}(2,3 \mathrm{C}) \mathrm{PdCl} 2 \mathrm{H}$
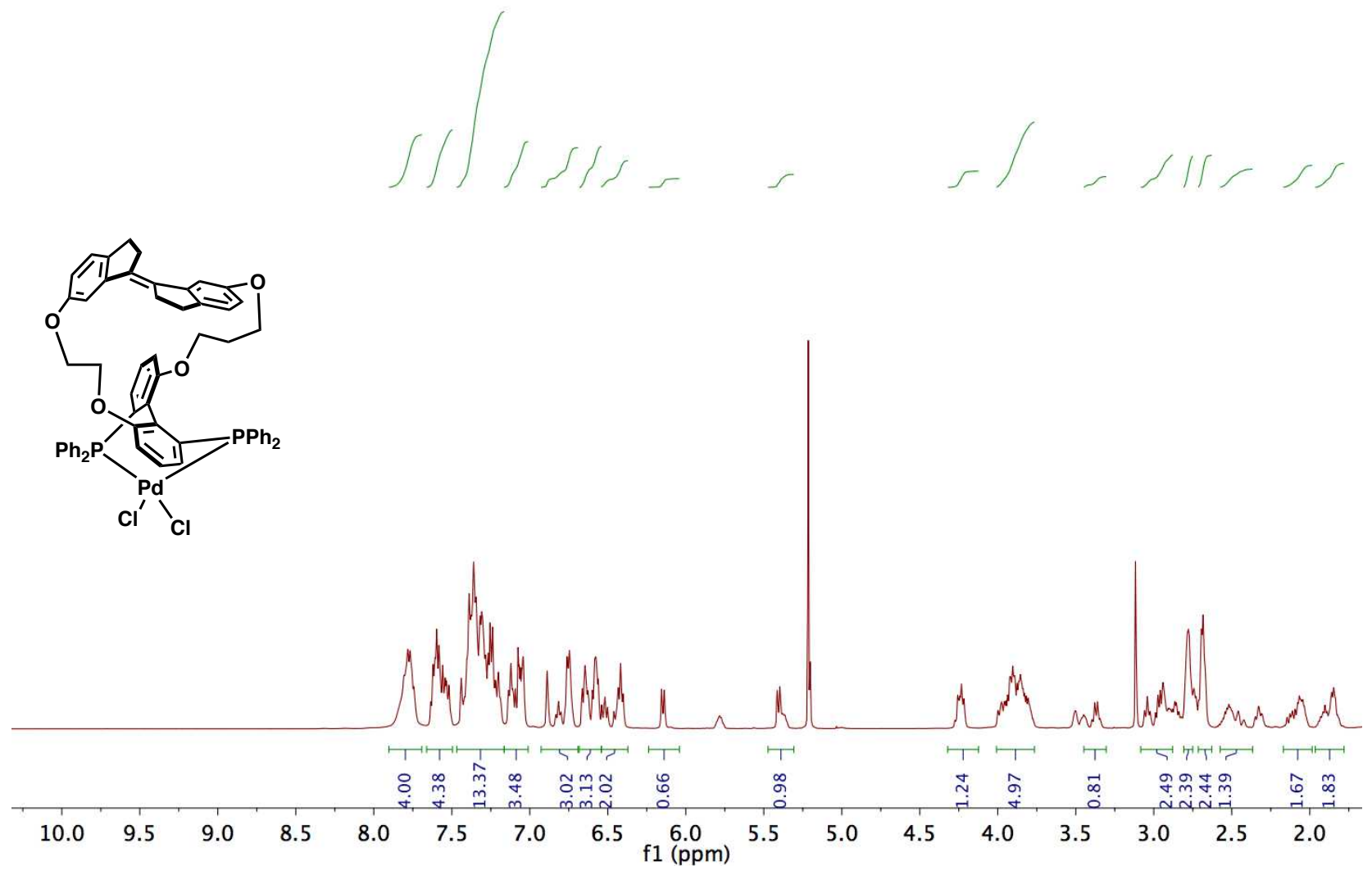

Figure S62. ${ }^{1} \mathrm{H}$ NMR spectrum of $E(2,3) \mathrm{PdCl}_{2}$. 


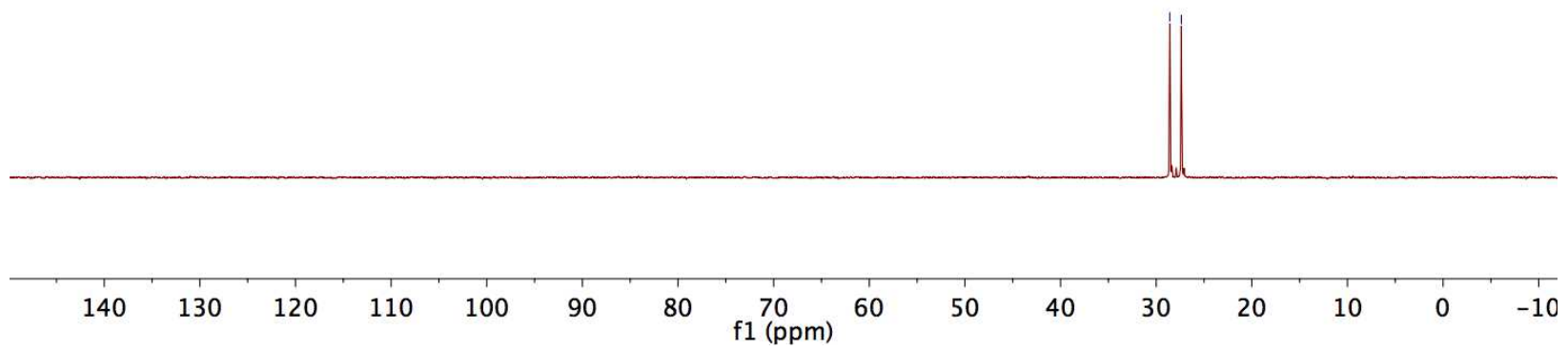

Figure S63. ${ }^{31} \mathrm{P}$ NMR spectrum of $E(2,3) \mathrm{PdCl}_{2}$. 


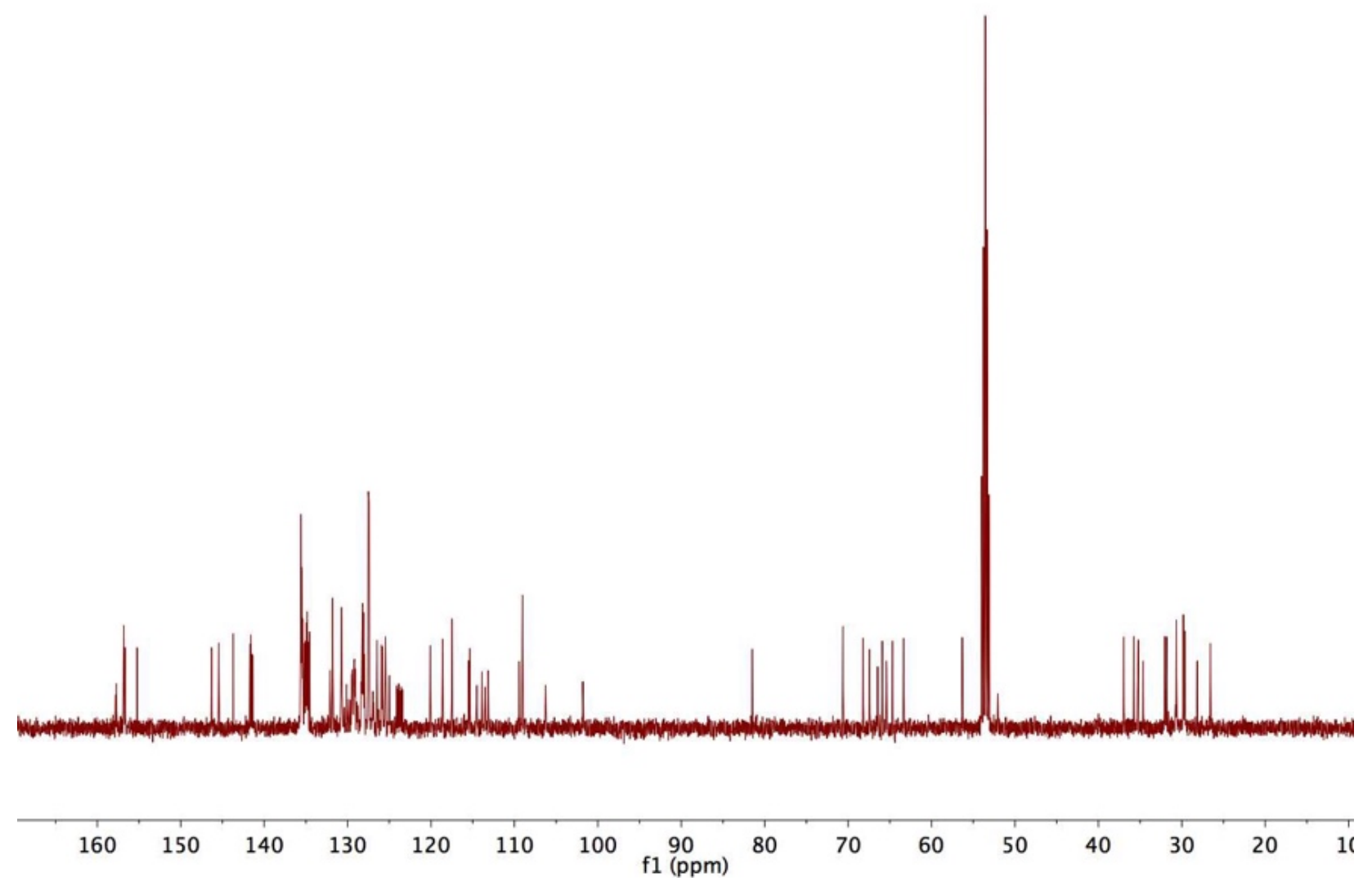

Figure S64. ${ }^{13} \mathrm{C}\left\{{ }^{1} \mathrm{H}\right\}$ NMR spectrum of $E(2,3) \mathrm{PdCl}$. 
$\mathrm{E}(3,3 \mathrm{C}) \mathrm{PdCl}{ }_{-} \mathrm{H}$
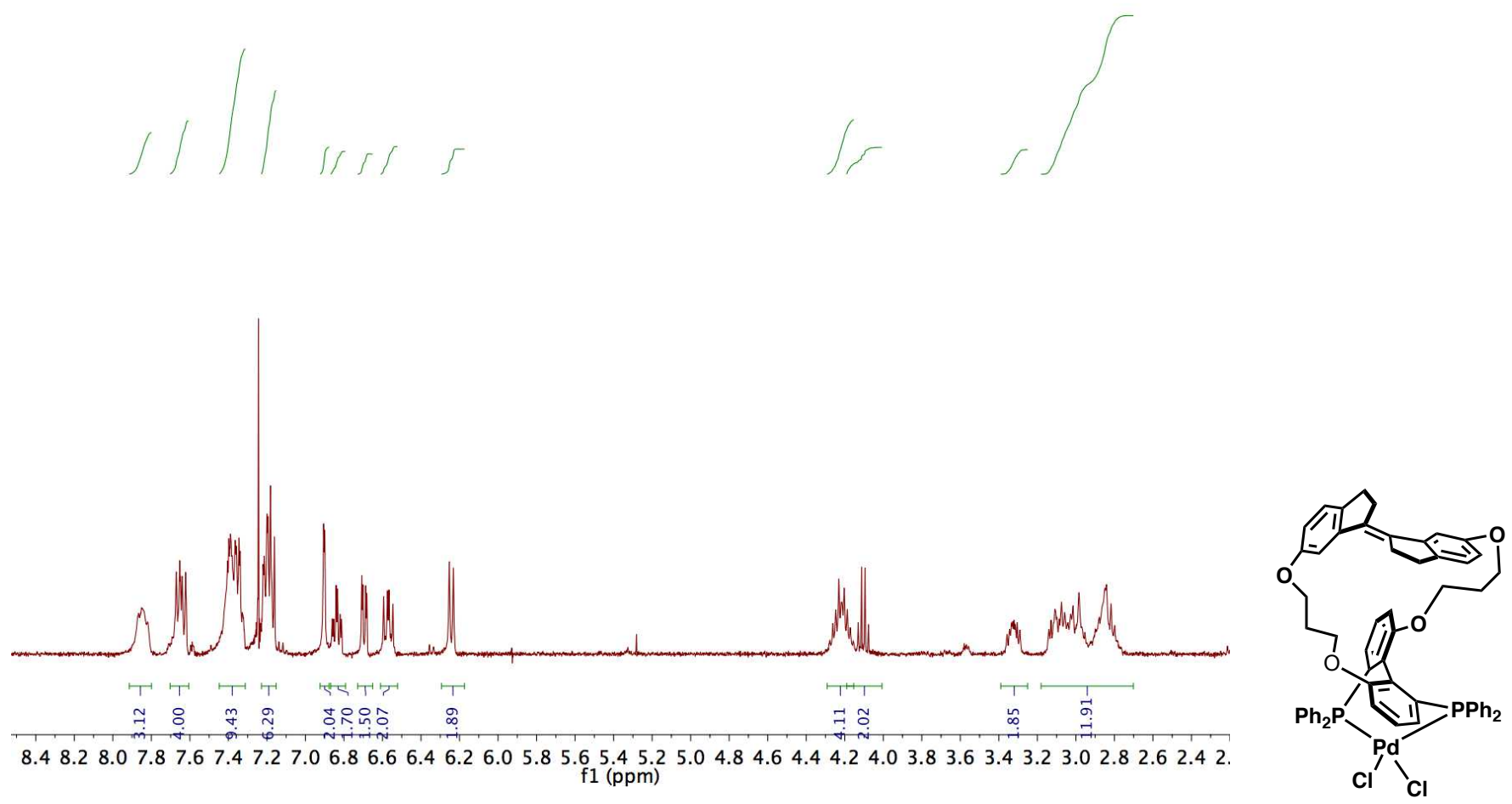

Figure S65. ${ }^{1} \mathrm{H}$ NMR spectrum of $E(3,3) \mathrm{PdCl}_{2}$. 
$E(3,3 C) P d C l 2 \_P$

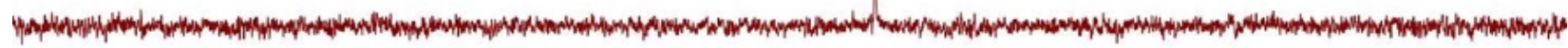

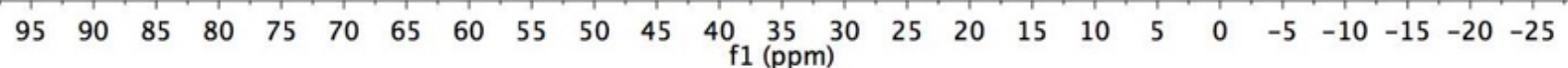

Figure S66. ${ }^{31} \mathrm{P}$ NMR spectrum of $E(3,3) \mathrm{PdCl}_{2}$. 
$\mathrm{E}(3,3 \mathrm{C}) \mathrm{PdCl} 2 \mathrm{C}$

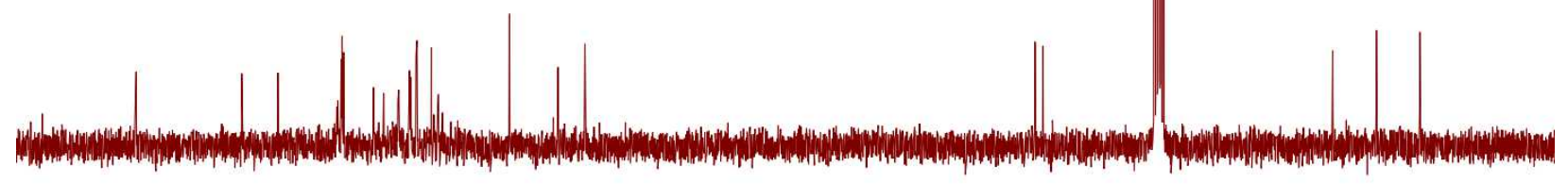

$\begin{array}{llllll}160 & 150 & 140 & 130 & 120 & 110\end{array}$

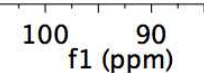

80

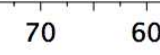

Figure S67. ${ }^{13} \mathrm{C}\left\{{ }^{1} \mathrm{H}\right\}$ NMR spectrum of $E(3,3) \mathrm{PdCl}_{2}$. 

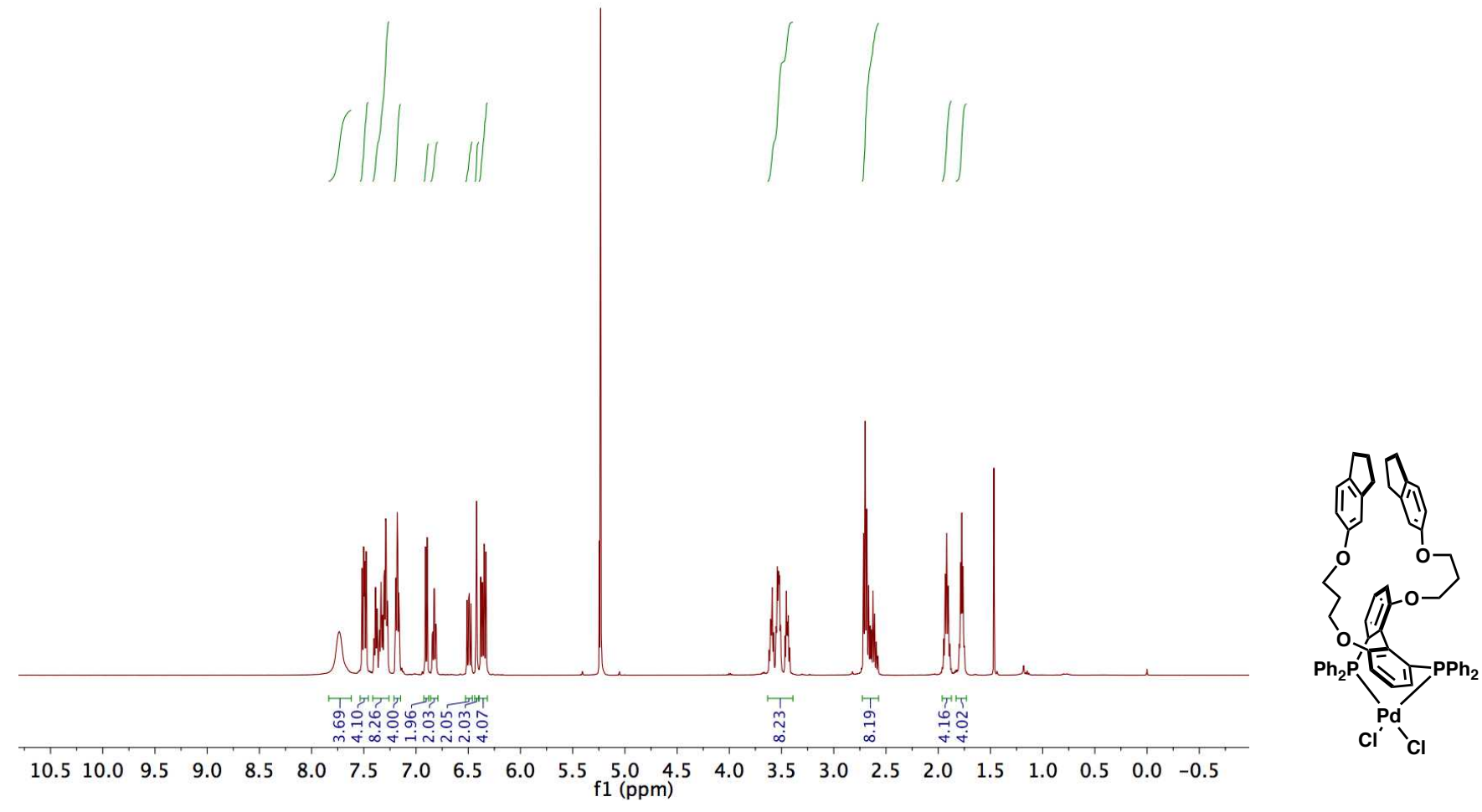

Figure S68. ${ }^{1} \mathrm{H}$ NMR spectrum of (Indane-Biphep)PdCl 2. 


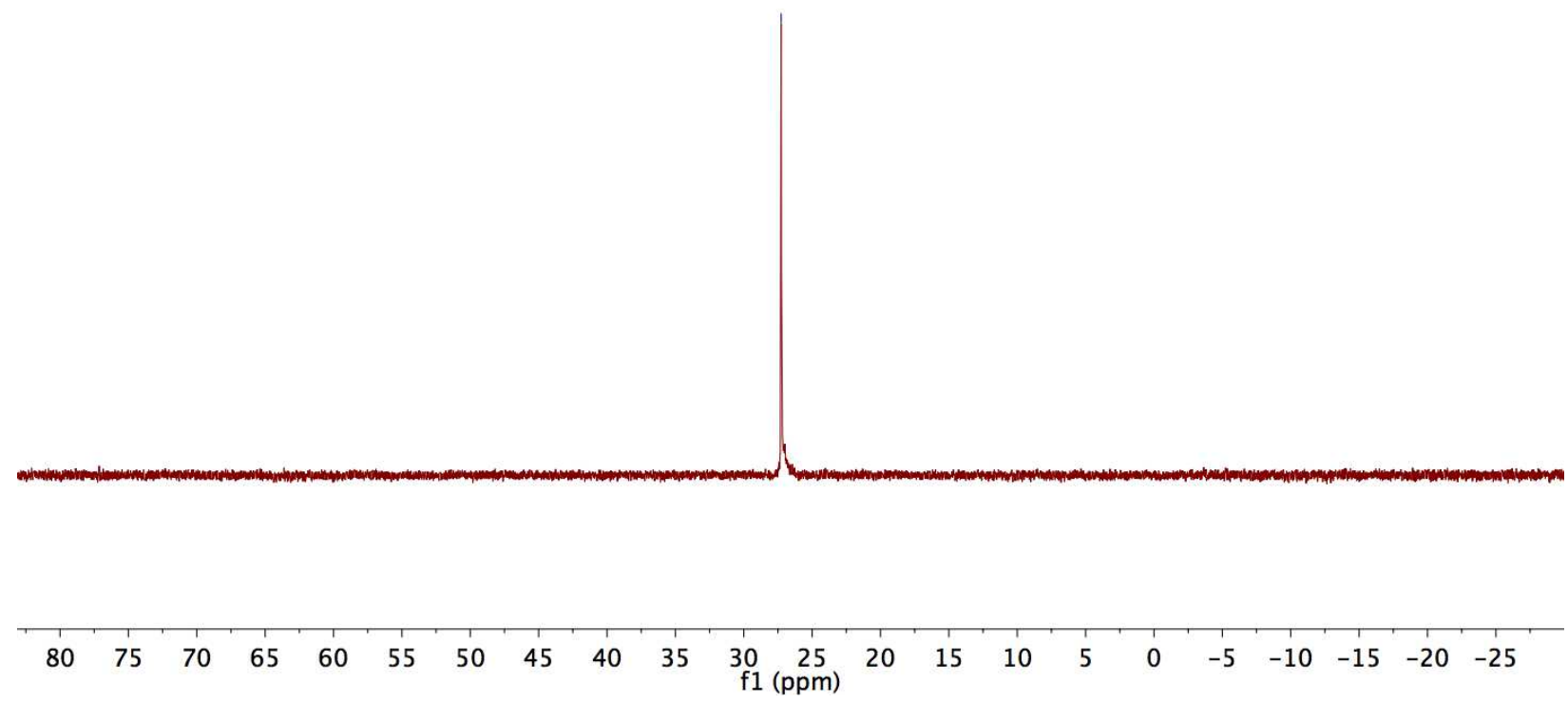

Figure S69. ${ }^{31} \mathrm{P}$ NMR spectrum of (Indane-Biphep) $\mathrm{PdCl}_{2}$. 


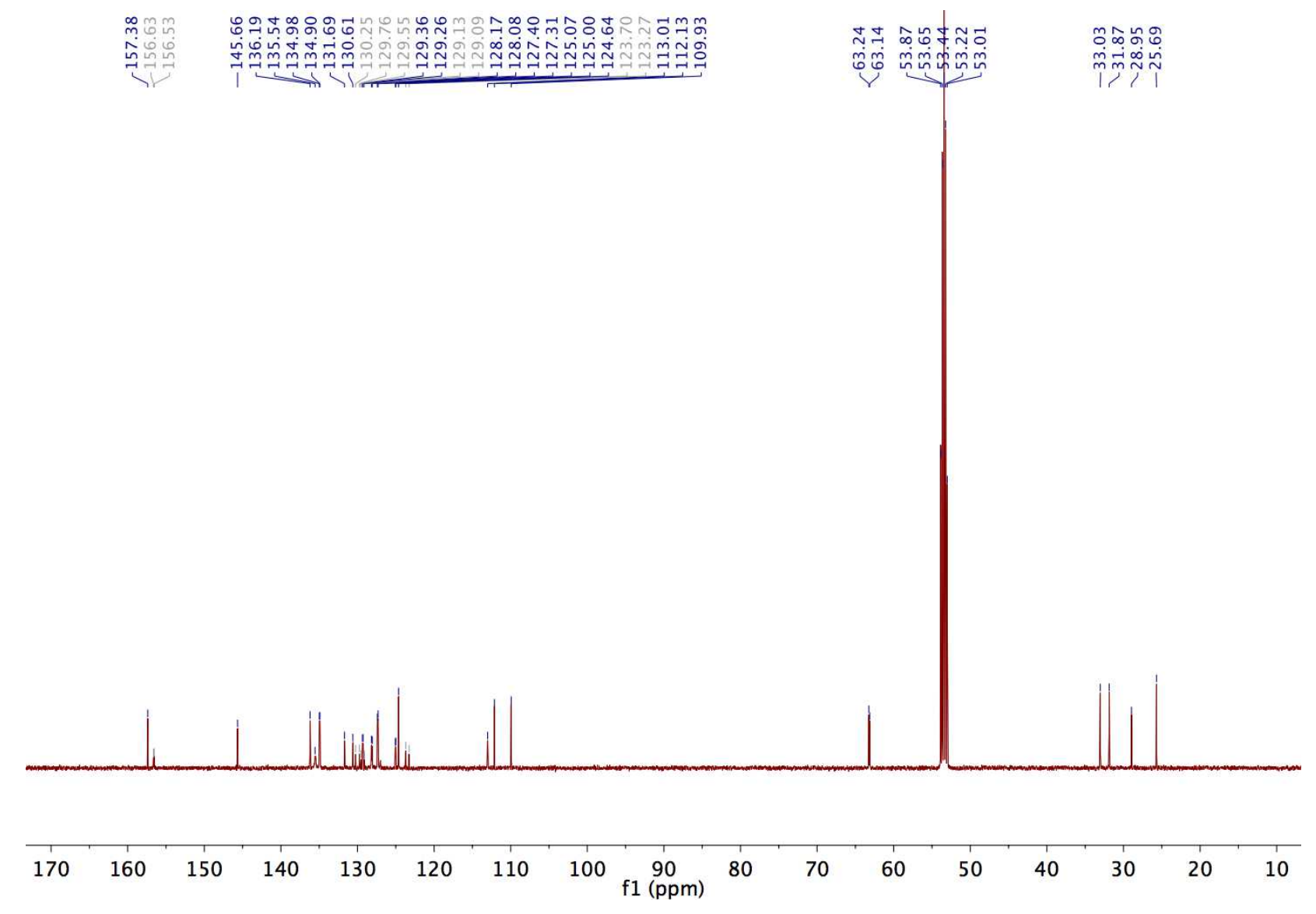

Figure S70. ${ }^{13} \mathrm{C}\left\{{ }^{1} \mathrm{H}\right\}$ NMR spectrum of (Indane-Biphep)PdCl 2 . 

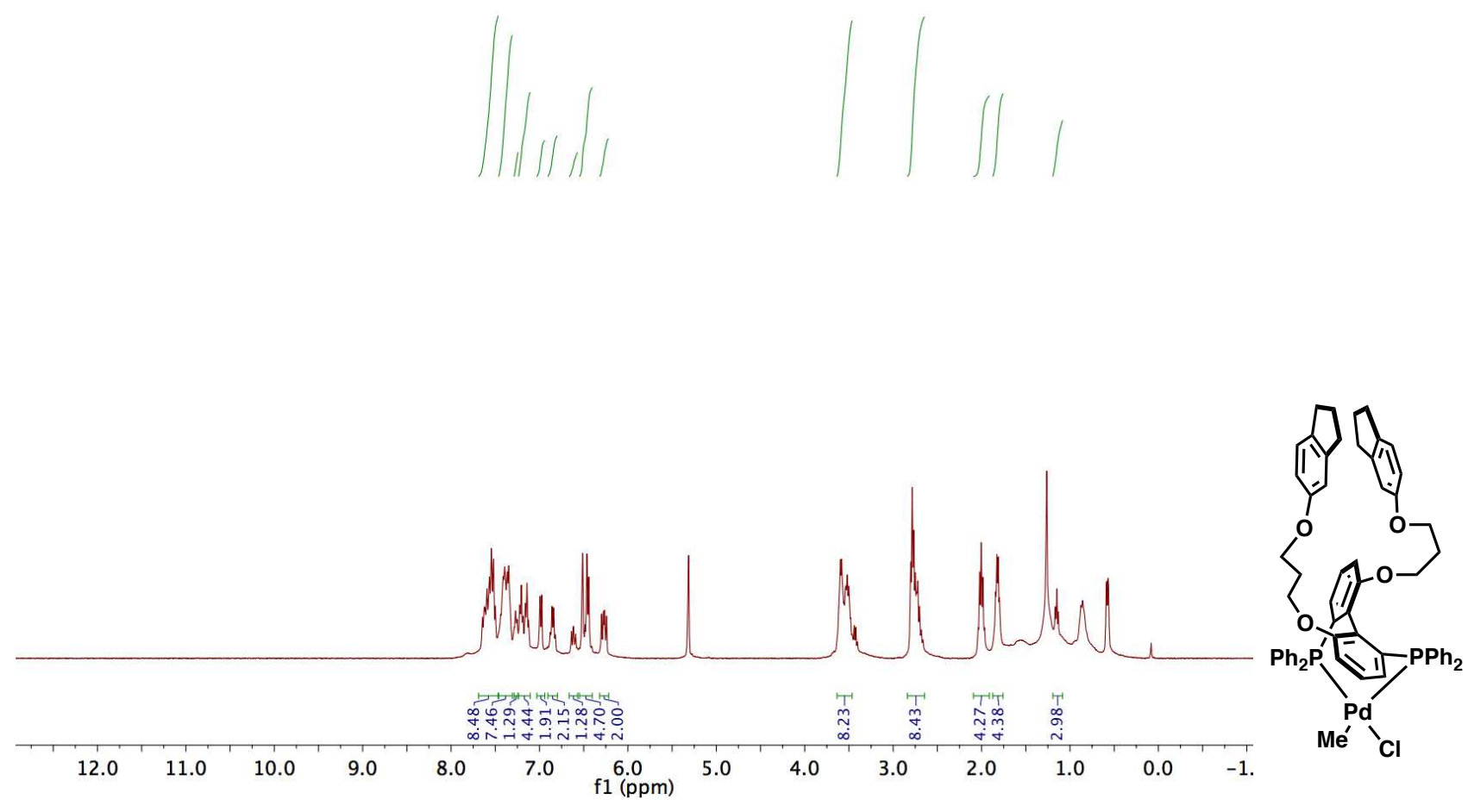

Figure S71. ${ }^{1} \mathrm{H}$ NMR spectrum of (Indane-Biphep)PdMeCl. 


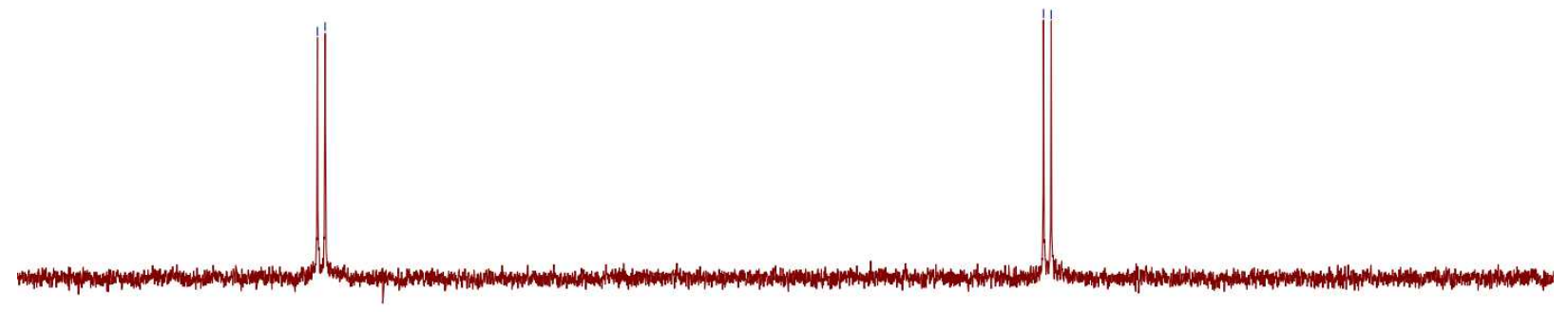
$\begin{array}{llllllllllllllllllllllllll}46 & 44 & 42 & 40 & 38 & 36 & 34 & 32 & 30 & 28 & 26 & 24 & \begin{array}{c}22 \\ \mathrm{f} 1(\mathrm{ppm})\end{array} & 20 & 18 & 16 & 14 & 12 & 10 & 8 & 6 & 4 & 2 & 0 & -2 & -\end{array}$

Figure S72. ${ }^{31} \mathrm{P}$ NMR spectrum of (Indane-Biphep)PdMeCl. 


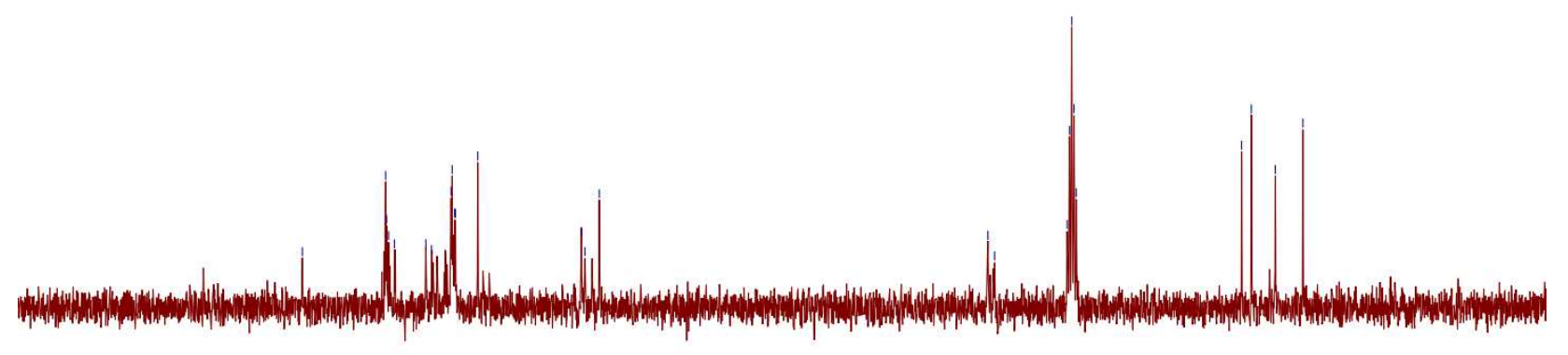

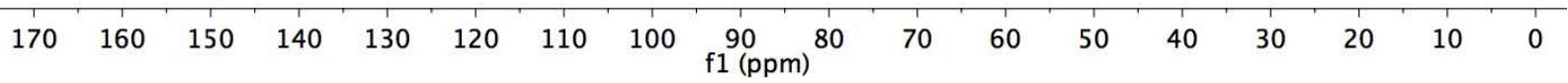

Figure S73. ${ }^{13} \mathrm{C}\left\{{ }^{1} \mathrm{H}\right\}$ NMR spectrum of (Indane-Biphep)PdMeCl.

\section{References}

(S1) Kean, Z. S.; Akbulatov, S.; Tian, Y.; Widenhoefer, R. A.; Boulatov, R.; Craig, S. L. Photomechanical actuation of ligand geometry in enantioselective catalysis. Angew. Chem. Int. Ed. 2014, 53, 14508.

(S2) Becker, J.; Orden, L.; White, P.; Gagne, M. Electron-Poor Benzonitriles as Labile, Stabilizing Ligands in Asymmetric Catalysis. Org. Lett. 2002, 4, 727.

(S3) Kucharski, T. J.; Huang, Z.; Yang, Q.-Z.; Tian, Y.; Rubin, N. C.; Concepcion, C. D.; Boulatov, R. Kinetics of thiol/disulfide exchange correlate weakly with the restoring force in the disulfide moiety. Angew. Chem. Int. Ed. 2009, 48, 7040. 
(S4) Zhang, Z.; Qian, H.; Longmire, J.; Zhang, X. Synthesis of Chiral Bisphosphines with Tunable Bite Angles and Their Applications in Asymmetric Hydrogenation of $\beta$-Ketoesters. J. Org. Chem. $2000,65,6223$.

(S5) The absence of detectable ${ }^{2} \mathrm{JPP}$ in these complexes was unexpected, but has been previously observed in group 10 complexes containing unsymmetric bis(phosphine) ligands: (a) Carty, A. J.; Johnson, D. K.; Jacobson, S. E. Synthesis and ${ }^{31} \mathrm{P}$ NMR Studies of Unsymmetrical cis-Diphosphinoalkenes and Their Complexes with Nickel(II), Palladium(II), and Platinum(II). J. Am. Chem. Soc. 1979, 101, 5612-5619; (b) King, R. B.; Cloyd, J. C. Poly (tertiary phosphines and arsines). XI. Phosphorus-31 Nuclear Magnetic Resonance Studies on Some Metal Complexes of Poly (tertiary phosphines). Inorg. Chem. 1975, 14, 1550-1554; (c) Verstuyft, A. W.; Redfield, D. A.; Cary, L. W.; Nelson, J. H. "Mixed-Ligand" Bis-Monodentate Phosphorus Donor Ligand Complexes of Palladium(II), $\mathrm{LL}^{\prime} \mathrm{PdCl}_{2} \mathrm{~A}$ Comprehensive Investigation. Inorg. Chem. 1976, 15, 1128-1133; (d) Segapelo, T. V.; Lillywhite, S.; Nordlander, E.; Haukka, M.; Darkwa, J. Palladium(II), platinum(II) and gold(I) complexes containing chiral diphosphines of the Josiphos and Walphos families - Synthesis and evaluation as anticancer agents. Polyhedron 2012, 36, 97-103.

(S6) (a) Amatore, C.; Azzabi, M.; Jutand, A. Stabilization of bis(triphenylphosphine)palladium(0) by chloride ions. Electrochemical generation of highly reactive zerovalent palladium complexes. J. Organomet. Chem. 1989, 363, C41. (b) Amatore, C.; Azzabi, M.; Jutand, A. Role and effects of halide ions on the rates and mechanisms of oxidative addition of iodobenzene to low-ligated zerovalent palladium complexes $\mathrm{PdO}\left(\mathrm{PPh}_{3}\right)_{2}$. J. Am. Chem. Soc. 1991, 113, 8375. (c) Amatore, C.; Jutand, A.; Suarez A. Intimate mechanism of oxidative addition to zerovalent palladium complexes in the presence of halide ions and its relevance to the mechanism of palladiumcatalyzed nucleophilic substitutions. J. Am. Chem. Soc. 1993, 115, 9531. (d) Amatore, C.; Azzabi, M.; Jutand, A. Rates and mechanism of the reversible oxidative addition of (Z)- and (E)-1,2dichloroethylene to low-ligated zerovalent palladium. J. Am. Chem. Soc. 1991, 113, 1670. 
(S7) Negishi, E.; King, A. O.; Okukado N. Selective carbon-carbon bond formation via transition metal catalysis. 3. A highly selective synthesis of unsymmetrical biaryls and diarylmethanes by the nickel- or palladium-catalyzed reaction of aryl- and benzylzinc derivatives with aryl halides. J. Org. Chem. 1977, 42, 1821.

(S8) Negishi, E.; Takahashi, T.; Akiyoshi, K. 'Bis(tripheny1phosphine)palladium :' Its Generation, Characterization, and Reactions J. Chem. Soc. Chem. Commun. 1986, 1338.

(S9) Frisch, M. J.; Trucks, G. W.; Schlegel, H. B.; Scuseria, G. E.; Robb, M. A. C., J. R.; Scalmani, G.; Barone, V.; Mennucci, B.; Petersson, G. A.; Nakatsuji, H.; Caricato, M.; Li, X.; Hratchian, H. P.; Izmaylov, A. F.; Bloino, J.; Zheng, G.; Sonnenberg, J. L.; Hada, M.; Ehara, M.; Toyota, K.; Fukuda, R.; Hasegawa, J.; Ishida, M.; Nakajima, T.; Honda, Y.; Kitao, O.; Nakai, H.; Vreven, T.; Montgomery, J. A., Jr.; Peralta, J. E.; Ogliaro, F.; Bearpark, M.; Heyd, J. J.; Brothers, E.; Kudin, K. N.; Staroverov, V. N.; Kobayashi, R.; Normand, J.; Raghavachari, K.; Rendell, A.; Burant, J. C.; Iyengar, S. S.; Tomasi, J.; Cossi, M.; Rega, N.; Millam, M. J.; Klene, M.; Knox, J. E.; Cross, J. B.; Bakken, V.; Adamo, C.; Jaramillo, J.; Gomperts, R.; Stratmann, R. E.; Yazyev, O.; Austin, A. J.; Cammi, R.; Pomelli, C.; Ochterski, J. W.; Martin, R. L.; Morokuma, K.; Zakrzewski, V. G.; Voth, G. A.; Salvador, P.; Dannenberg, J. J.; Dapprich, S.; Daniels, A. D.; Farkas, Ö.; Foresman, J. B.; Ortiz, J. V.; Cioslowski, J.; Fox, D. J. 2010.

(S10) Akbulatov, S.; Tian, Y.; Huang, Z.; Kucharski, T. J.; Yang, Q.; Boulatov, R. Experimentally realized mechanochemistry distinct from force-accelerated scission of loaded bonds. Science. 2017, 357, 299.

(S11) Bai, T.; Xue, L.; Xue, P.; Zhu, J.; Sung, H.; Ma, S.; Wiliams, I. D.; Lin, Z.; Jia, G. Insertion Reactions of Allenes with Palladium Aryl Complexes $\left[\mathrm{Pdl}(\mathrm{Ph})\left(\mathrm{PPh}_{3}\right)\right]_{2}$ and $\mathrm{Pdl}(\mathrm{Ph})(\mathrm{dppe})$. Organometallics 2008, 27, 2614.

(S12) Kalek, M.; Stawinski, J. Palladium-Catalyzed C-P Bond Formation: Mechanistic Studies on the Ligand Substitution and the Reductive Elimination. An Intramolecular Catalysis by the Acetate Group in Pdll Complexes. Organometallics 2008, 27, 5876. 
(S13) Dierkes, P.; van Leeuwen, P. W. N. M., The Bite Angle Makes the Difference: A Practical Ligand Parameter for Diphosphine Ligands. Dalton Trans. 1999, 1519. 